\title{
Stochastic Stabilization of Slender Beams in Space: Modeling and Boundary Control
}

\author{
K. D. Do and A. D. Lucey \\ Department of Mechanical Engineering, Curtin University, Kent Street, Bentley, WA 6102, Australia
}

\begin{abstract}
This paper considers the problem of modeling and boundary feedback stabilization of extensible and shearable slender beams with large deformations and large rotations in space under both deterministic and stochastic loads induced by flows. Fully nonlinear equations of motion of the beams are first derived. Boundary feedback controllers are then designed for global practical exponential $p$-stabilization of the beams based on the Lyapunov direct method. A new Lyapunov-type theorem is developed to study well-posedness and stability of stochastic evolution systems (SESs) in Hilbert space.
\end{abstract}

Key words: Slender beams; Large motions; Boundary control; Stochastic evolution system; Well-posedness; p-stability.

\section{Introduction}

This paper focuses on relatively slender beams, for which the shear magnitude is smaller than that of the spatial gradient of the transverse displacements. Due to their large length-to-diameter ratio, extensibility and shearability, the relatively slender beams exhibit both large and small motions (both deflection and rotation) under external (both deterministic and stochastic) loads. Although large motions can cause a serious failure (loop formation or hockling) in beams, most of existing boundary control works (e.g., [5,6,12,13,17,20,22-25,25,26,28, 38-41,44,48,50] based on the Lyapunov direct and flatness methods, and $[4,32,33]$ based on the backstepping method on single beams, and $[15,27,29,35]$ on multiple beams) on boundary control have considered only small deflection (vibration). None of the equations of motion in the above works can describe loop formation due to the fact that they are obtained by linearizing the axial stretch and rotational motions, and neglecting the shear strains, see [16,37], and therefore exclude large motions.

Boundary control of slender beams with large motions has received less attention. In $[8,14]$ (see also $[2,31]$ for models of slender beams, where only large deflection is considered), boundary control of unshearable risers/beams with large deflections was considered. Boundary control of extensible and shearable slender beams has been considered in [9] in three-dimensional space (3D). In these works, the external loads are assumed to be deterministic except for the work in [11], where

Email address: duc@curtin.edu.au,

T.Lucey@curtin.edu. au (K. D. Do and A. D. Lucey). stochastic external loads are initially considered for slender beams in two-dimensional space (2D). Control design and stability analysis for stochastic beams is much harder than for deterministic beams. For example, the stochastic component of flows, which enters to the beam system via the hydrodynamic/aerodynamic Centripetal matrix, potentially destabilizes the beam system under deterministic control designs.

The main contributions of this paper consist of three parts. First, fully nonlinear equations of motion of the beams and their properties are derived in an appropriate form for boundary control design by using deformation theory and sea loads on offshore structures. The unit quaternion is used for attitude representation of the beams to resolve singularities caused by Euler angles. Second, boundary feedback controllers are designed for global practical exponential stabilization of the beams based on the Lyapunov direct method. In the control design, various Young's and Hölder's inequalities and Sobolev embedding, a flexible combination of Earthfixed and body-fixed coordinates, and cross vector products are used. Third, a new Lyapunov-type theorem is developed to study well-posedness and stability analysis of a class of nonlinear SESs in Hilbert space. This theorem does not require global monotonic and linear growth conditions as in e.g., [7,36,46,47]. The Lyapunov function uses $\|\cdot\|_{V}$ instead of $\|.\|_{H}$ as in [12]. This allows to study well-posedness of SESs, for which it is difficult to apply multiple Gelfand triples because $V \subset H$.

Notations. The symbols $\wedge$ and $\vee$ denote the infimum and supremum operators, respectively. The symbols "col", "X", "E" denote the column operator, vector cross product operator, and the expected value, respectively. 


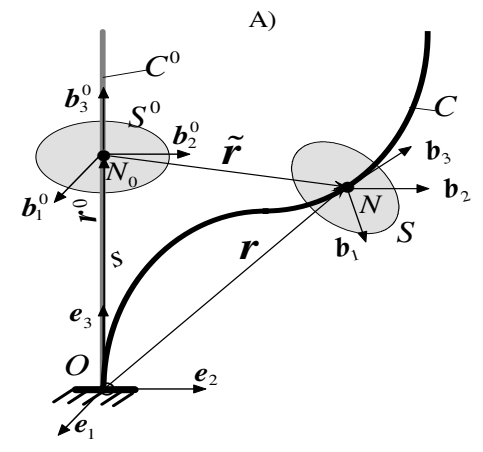

B)

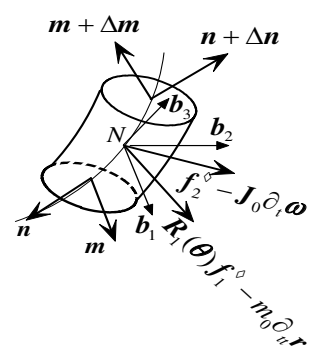

Fig. 1. A) Deformation geometry of the beam; B) Forces and moments acting on a beam element.

\section{Mathematical model}

We assume that plane sections are rigid; and the beam material is elastic, homogeneous and isotropic. Equations of motion are briefly derived, see [9].

\subsection{Kinematics}

The reference configuration $\mathcal{B}^{0}$ is described by the position of the base straight line $C^{0}$ parameterized by its arclength coordinate $s$ and the fixed basis $\left(\boldsymbol{b}_{1}^{0}, \boldsymbol{b}_{2}^{0}, \boldsymbol{b}_{3}^{0}\right)$, where $\left(\boldsymbol{b}_{1}^{0}, \boldsymbol{b}_{2}^{0}\right)$ are the principal axes of inertia of the cross section $S^{0}(s)$ through $N^{0}$, see Fig. 1A. Thus, $C^{0}$ is described by $\boldsymbol{r}^{0}(s)=0 \boldsymbol{e}_{1}+0 \boldsymbol{e}_{2}+\boldsymbol{s}_{3}$. We denote by $\Gamma$ the beam length in its reference state. The actual configuration $\mathcal{B}$ of the curved beam is described by the actual position of the base curve $C$ and the actual configuration $S$ of cross sections through $N$. The base curve is described by $\boldsymbol{r}$ while the material cross section is described by the unit vectors $\left\{\boldsymbol{b}_{1}, \boldsymbol{b}_{2}, \boldsymbol{b}_{3}\right\}$ with $\boldsymbol{b}_{3}$ being aligned with $\boldsymbol{r}_{s}$ and $\boldsymbol{b}_{3}=\boldsymbol{b}_{1} \times \boldsymbol{b}_{2}$. The deformation from $\mathcal{B}^{0}$ to $\mathcal{B}$ is achieved by means of the vector $\boldsymbol{r}=r_{1} \boldsymbol{b}_{1}+r_{2} \boldsymbol{b}_{2}+r_{3} \boldsymbol{b}_{3}$, and the orthogonal tensor $\boldsymbol{R}_{1}(\boldsymbol{\theta})$ describing the incremental rigid rotation suffered by $S^{0}$ so that $\boldsymbol{b}_{k}=\boldsymbol{R}_{1}(\boldsymbol{\theta}) \boldsymbol{b}_{k}^{0}, k=1,2,3$ via the sequence $\theta_{1} \rightarrow \theta_{2} \rightarrow \theta_{3}$. This gives $\boldsymbol{b}_{k s}=\boldsymbol{R}_{1 s} \boldsymbol{b}_{k}^{0}$, where $\boldsymbol{R}_{1 s}=$ $\boldsymbol{\mu} \times \boldsymbol{b}_{k}$ with $\boldsymbol{\mu}$ the axial vector of $\boldsymbol{R}_{1 s} \boldsymbol{R}_{1}^{T}$. The generalized strains (the stretch $\varepsilon$ and shear strains $\eta_{1}$ and $\eta_{2}$ ) are expressed by the stretch vector $\boldsymbol{\nu}=\eta_{1} \boldsymbol{b}_{1}+\eta_{2} \boldsymbol{b}_{2}+(1+\varepsilon) \boldsymbol{b}_{3}$ in its local basis: $\boldsymbol{\nu}=\boldsymbol{r}_{s}$. Thus,

$$
\begin{aligned}
& \boldsymbol{r}_{s}=\eta_{1} \boldsymbol{b}_{1}+\eta_{2} \boldsymbol{b}_{2}+(1+\varepsilon) \boldsymbol{b}_{3} \\
& \boldsymbol{\mu}=\mu_{1} \boldsymbol{b}_{1}+\mu_{2} \boldsymbol{b}_{2}+\mu_{3} \boldsymbol{b}_{3}, \boldsymbol{\omega}=\omega_{1} \boldsymbol{b}_{1}+\omega_{2} \boldsymbol{b}_{2}+\omega_{3} \boldsymbol{b}_{3}, \\
& \boldsymbol{b}_{k s}=\boldsymbol{\mu} \times \boldsymbol{b}_{k}, \boldsymbol{b}_{k t}=\boldsymbol{\omega} \times \boldsymbol{b}_{k},\left(\boldsymbol{\mu} \times \boldsymbol{b}_{k}\right)_{t}=\left(\boldsymbol{\omega} \times \boldsymbol{b}_{k}\right)_{s} .
\end{aligned}
$$

From the above derivation, we have

$$
\begin{aligned}
& \operatorname{col}\left(\eta_{1}, \eta_{2}, \varepsilon\right)=\boldsymbol{R}_{1}^{T}(\boldsymbol{\theta}) \boldsymbol{r}_{s}-\boldsymbol{r}_{s}^{0}, \\
& \operatorname{col}\left(\mu_{1}, \mu_{2}, \mu_{3}\right)=\boldsymbol{R}_{2}^{-1}(\boldsymbol{\theta}) \operatorname{col}\left(\theta_{1 s}, \theta_{2 s}, \theta_{3 s}\right), \\
& \operatorname{col}\left(\omega_{1}, \omega_{2}, \omega_{3}\right)=\boldsymbol{R}_{2}^{-1}(\boldsymbol{\theta}) \operatorname{col}\left(\theta_{1 t}, \theta_{2 t}, \theta_{3 t}\right),
\end{aligned}
$$

where $\boldsymbol{R}_{2}(\boldsymbol{\theta})$ is the transformation matrix that relates the angular vector $\boldsymbol{\omega}$ in the local basis to the Euler rate vector $\boldsymbol{\theta}_{t}$. When $\theta_{2}= \pm \frac{\pi}{2}$, there are singularities in (2). Thus, we use the unit quaternion vector $\boldsymbol{q}=\operatorname{col}\left(q_{1}, q_{2}, q_{3}, q_{4}\right)$ for attitude representation with $\|\boldsymbol{q}\|^{2}=1$ relating to $\left(\theta_{1}, \theta_{2}, \theta_{3}\right)$ via the sequence $\theta_{1} \rightarrow$ $\theta_{2} \rightarrow \theta_{3}$, see [34]. Thus, $\boldsymbol{R}_{1}$ is given in terms of $\boldsymbol{q}$ as:

$$
\boldsymbol{R}_{1}(\boldsymbol{q})=\boldsymbol{I}_{3}+2 q_{1} \boldsymbol{S}(\overline{\boldsymbol{q}})+2 \boldsymbol{S}^{2}(\overline{\boldsymbol{q}})
$$

where $\overline{\boldsymbol{q}}:=\operatorname{col}\left(q_{2}, q_{3}, q_{4}\right)$ and the matrix $\boldsymbol{S}(\boldsymbol{x})$ is defined as $\boldsymbol{S}(\boldsymbol{x}) \boldsymbol{y}=\boldsymbol{x} \times \boldsymbol{y}$ for all $(\boldsymbol{x}, \boldsymbol{y}) \in \mathbb{R}^{3}$. Let us also define:

$$
\boldsymbol{K}(\boldsymbol{q})=\frac{1}{2}\left[\begin{array}{l}
-\overline{\boldsymbol{q}}^{T} \\
q_{1} \boldsymbol{I}_{3}+\boldsymbol{S}(\overline{\boldsymbol{q}})
\end{array}\right] .
$$

\subsection{Kinetic}

Balancing linear and angular momentum on a beam element, see Fig.1B, gives the equations of motion:

$$
\begin{aligned}
& m_{0} \tilde{\boldsymbol{r}}_{t t}=\boldsymbol{n}_{s}+\boldsymbol{R}_{1}(\boldsymbol{\theta}) \boldsymbol{f}_{1}^{\diamond}, \\
& \boldsymbol{J}_{0} \boldsymbol{\omega}_{t}=\boldsymbol{m}_{s}+\boldsymbol{r}_{s} \times \boldsymbol{n}-\boldsymbol{\omega} \times \boldsymbol{J}_{0} \boldsymbol{\omega}+\boldsymbol{f}_{2}^{\diamond},
\end{aligned}
$$

where $m_{0}$ is the beam mass per unit length; $\boldsymbol{J}_{0}$ is the mass moment matrix of inertia; $\boldsymbol{n}$ and $\boldsymbol{m}$ denote the contact force and moment vectors; and (see Fig. 1A)

$$
\tilde{\boldsymbol{r}}=\boldsymbol{r}-\boldsymbol{r}^{0} \text {. }
$$

a) Nonconservative forces and moments $\boldsymbol{f}_{1}^{\diamond}$ and $\boldsymbol{f}_{2}^{\diamond}$ : Let $\boldsymbol{v}$ be the linear velocity vector in the local basis, i.e.,

$$
\boldsymbol{v}=\boldsymbol{R}_{1}^{-1}(\boldsymbol{\theta}) \tilde{\boldsymbol{r}}_{t}
$$

Let $\boldsymbol{v}_{f}$ and $\boldsymbol{\omega}_{f}$ be the linear and angular velocity vectors of the fluid passing the beam at $(s, t)$. The total generalized force vector induced by the fluid acting on the beam denoted by $\boldsymbol{f}^{\diamond}:=\operatorname{col}\left(\boldsymbol{f}_{1}^{\diamond}, \boldsymbol{f}_{2}^{\diamond}\right)$ in the local basis is [18]:

$$
\boldsymbol{f}^{\diamond}=\underbrace{\boldsymbol{M}_{A}^{\diamond} \boldsymbol{v}_{r t}^{\diamond}+\boldsymbol{C}_{A}^{\diamond}\left(\boldsymbol{v}_{r}^{\diamond}\right) \boldsymbol{v}_{r}^{\diamond}}_{\text {Added mass }}+\underbrace{\boldsymbol{D}_{A}^{\diamond}\left(\boldsymbol{v}_{r}^{\diamond}\right) \boldsymbol{v}_{r}^{\diamond}}_{\text {Damping }}+\underbrace{\boldsymbol{f}_{0}^{\diamond}(s, t)}_{\text {Other loads }},
$$

where

$$
\begin{aligned}
& \boldsymbol{v}_{r}^{\diamond}=\operatorname{col}\left(\boldsymbol{v}_{r}, \boldsymbol{\omega}_{r}\right), \boldsymbol{v}_{r}=\boldsymbol{v}-\boldsymbol{v}_{f}, \boldsymbol{\omega}=\boldsymbol{\omega}-\boldsymbol{\omega}_{f}, \\
& \boldsymbol{f}_{0}^{\diamond}=\operatorname{col}\left(\boldsymbol{f}_{10}^{\diamond}, \boldsymbol{f}_{20}^{\diamond}\right), \boldsymbol{M}_{A}^{\diamond}=\operatorname{diag}\left(\boldsymbol{M}_{A}, \boldsymbol{J}_{A}\right) \text {, } \\
& \boldsymbol{D}_{A}^{\diamond}\left(\boldsymbol{v}_{r}^{\diamond}\right)=\operatorname{diag}\left(\boldsymbol{D}_{A 1}\left(\boldsymbol{v}_{r}\right), \boldsymbol{D}_{A 2}\left(\boldsymbol{\omega}_{r}\right)\right) \text {, } \\
& \boldsymbol{C}_{A}^{\diamond}\left(\boldsymbol{v}_{r}^{\diamond}\right) \boldsymbol{v}_{r}^{\diamond}=\operatorname{col}\left(\boldsymbol{\omega}_{r} \times\left(\boldsymbol{M}_{A} \boldsymbol{v}_{r}\right), \boldsymbol{\omega}_{r} \times\left(\boldsymbol{J}_{A} \boldsymbol{\omega}_{r}\right)\right. \\
& \left.+\boldsymbol{v}_{r} \times\left(\boldsymbol{M}_{A} \boldsymbol{v}_{r}\right)\right), \\
& \boldsymbol{D}_{A 1}\left(\boldsymbol{v}_{r}\right)=\boldsymbol{D}_{A 11}+\boldsymbol{D}_{A 12} \boldsymbol{v}_{r} \otimes \boldsymbol{v}_{r}, \\
& \boldsymbol{D}_{A 2}\left(\boldsymbol{\omega}_{r}\right)=\boldsymbol{D}_{A 21}+\boldsymbol{D}_{A 22} \boldsymbol{\omega}_{r} \otimes \boldsymbol{\omega}_{r},
\end{aligned}
$$

where the added mass and added inertia matrices $\boldsymbol{M}_{A}$ and $\boldsymbol{J}_{A}$ are diagonal and negative definite; $\boldsymbol{D}_{A 1}\left(\boldsymbol{v}_{r}\right)$ and $\boldsymbol{D}_{A 2}\left(\boldsymbol{\omega}_{r}\right)$ are damping matrices; $\boldsymbol{D}_{A i j},(i, j)=1,2$ are diagonal and negative definite matrices; and the operator $\otimes$ is defined as $\boldsymbol{a} \otimes \boldsymbol{a}:=\operatorname{diag}\left(a_{1}^{2}, a_{2}^{2}, a_{3}^{2}\right)$ with $\boldsymbol{a}=\operatorname{col}\left(a_{1}, a_{2}, a_{3}\right)$. In general, $\boldsymbol{v}_{f}, \boldsymbol{\omega}_{f}$ and $\boldsymbol{f}_{i 0}^{\diamond}$ with $i=1,2$ consist of deterministic components $\overline{\boldsymbol{v}}_{f}, \overline{\boldsymbol{\omega}}_{f}, \overline{\boldsymbol{f}}_{i 0}^{\diamond}$, and stochastic ones $\tilde{\boldsymbol{v}}_{f}, \tilde{\boldsymbol{\omega}}_{f}, \tilde{\boldsymbol{f}}_{i 0}^{\diamond}$. Thus, we can write

$$
\begin{aligned}
& \boldsymbol{v}_{f}=\overline{\boldsymbol{v}}_{f}+\tilde{\boldsymbol{v}}_{f}, \boldsymbol{\omega}_{f}=\overline{\boldsymbol{\omega}}_{f}+\tilde{\boldsymbol{\omega}}_{f}, \\
& \boldsymbol{f}_{10}^{\diamond}=\overline{\boldsymbol{f}}_{10}^{\diamond}+\tilde{\boldsymbol{f}}_{10}^{\diamond}, \boldsymbol{f}_{20}^{\diamond}=\overline{\boldsymbol{f}}_{20}^{\diamond}+\tilde{\boldsymbol{f}}_{20}^{\diamond} .
\end{aligned}
$$


b) Equations of motion: With (2)-(10), we can write (5) as the stochastic PDEs:

$$
\begin{aligned}
d \tilde{\boldsymbol{r}} & =\boldsymbol{R}_{1}(\boldsymbol{q}) \boldsymbol{v} d t, \quad d \boldsymbol{q}=\boldsymbol{K}(\boldsymbol{q}) \boldsymbol{\omega} d t \\
d \boldsymbol{v} & =\boldsymbol{M}^{-1}\left[-\boldsymbol{\omega} \times(\boldsymbol{M} \boldsymbol{v})+\boldsymbol{R}_{1}^{-1}(\boldsymbol{q}) \boldsymbol{n}_{s}+\boldsymbol{D}_{A 11} \boldsymbol{v}\right. \\
& \left.+\boldsymbol{D}_{A 12} \boldsymbol{v} \otimes \boldsymbol{v} \boldsymbol{v}+\boldsymbol{f}_{1}\right] d t+\boldsymbol{M}^{-1} \boldsymbol{G}_{1}(\boldsymbol{v}, \boldsymbol{\omega}) \boldsymbol{\Delta}_{1} d \boldsymbol{W}_{1}, \\
d \boldsymbol{\omega} & =\boldsymbol{J}^{-1}\left[\boldsymbol{m}_{s}-\boldsymbol{\omega} \times(\boldsymbol{J} \boldsymbol{\omega})+\boldsymbol{r}_{s} \times \boldsymbol{n}+\boldsymbol{v} \times\left(\boldsymbol{M}_{A} \boldsymbol{v}\right)\right. \\
& \left.+\boldsymbol{D}_{A 21} \boldsymbol{\omega}+\boldsymbol{D}_{A 22} \boldsymbol{\omega} \otimes \boldsymbol{\omega} \boldsymbol{\omega}+\boldsymbol{f}_{2}\right] d t+\boldsymbol{G}_{2}(\boldsymbol{v}, \boldsymbol{\omega}) \boldsymbol{\Delta}_{2} d \boldsymbol{W}_{2},
\end{aligned}
$$

where $\boldsymbol{M}=\boldsymbol{I}_{3} m_{0}-\boldsymbol{M}_{A}, \boldsymbol{J}=\boldsymbol{J}_{0}-\boldsymbol{J}_{A}, \boldsymbol{\Delta}_{i}$ with $i=1,2$ are covariance matrices of the standard Wiener process vectors $\boldsymbol{W}_{i}$, which model $\tilde{\boldsymbol{f}}_{i 0}^{\diamond}, \tilde{\boldsymbol{v}}_{f}$ and $\tilde{\boldsymbol{\omega}}_{f}$, and

$$
\begin{aligned}
\boldsymbol{f}_{1}=-\boldsymbol{\omega} \times\left(\boldsymbol{M}_{A} \overline{\boldsymbol{v}}_{f}\right)-\overline{\boldsymbol{\omega}}_{f} \times\left(\boldsymbol{M}_{A} \boldsymbol{v}\right)-3 \boldsymbol{D}_{A 12} \boldsymbol{v} \otimes \boldsymbol{v} \overline{\boldsymbol{v}}_{f} \\
\quad+3 \boldsymbol{D}_{A 12} \overline{\boldsymbol{v}}_{f} \otimes \overline{\boldsymbol{v}}_{f} \boldsymbol{v}+\overline{\boldsymbol{f}}_{10}, \\
\boldsymbol{f}_{2}=-\boldsymbol{\omega} \times\left(\boldsymbol{J}_{A} \overline{\boldsymbol{\omega}}_{f}\right)-\overline{\boldsymbol{\omega}}_{f} \times\left(\boldsymbol{J}_{A} \boldsymbol{\omega}\right)-\boldsymbol{v} \times\left(\boldsymbol{M}_{A} \overline{\boldsymbol{v}}_{f}\right)+\overline{\boldsymbol{f}}_{20} \\
\quad-\overline{\boldsymbol{v}}_{f} \times\left(\boldsymbol{M}_{A} \boldsymbol{v}\right)-3 \boldsymbol{D}_{A 22} \boldsymbol{\omega} \otimes \boldsymbol{\omega} \overline{\boldsymbol{\omega}}_{f}+3 \boldsymbol{D}_{A 22} \overline{\boldsymbol{\omega}}_{f} \otimes \overline{\boldsymbol{\omega}}_{f} \boldsymbol{\omega}, \\
\boldsymbol{G}_{1}(\boldsymbol{v}, \boldsymbol{\omega})=\operatorname{row}\left(\boldsymbol{G}_{11}, \boldsymbol{G}_{12}, \boldsymbol{G}_{13}, \boldsymbol{G}_{14}, \boldsymbol{G}_{15}, \boldsymbol{G}_{16}\right), \\
\boldsymbol{G}_{2}(\boldsymbol{v}, \boldsymbol{\omega})=\operatorname{row}\left(\boldsymbol{G}_{21}, \boldsymbol{G}_{22}, \boldsymbol{G}_{23}, \boldsymbol{G}_{24}, \boldsymbol{G}_{25}, \boldsymbol{G}_{26}, \boldsymbol{G}_{27}, \boldsymbol{G}_{28}\right),
\end{aligned}
$$

with

$$
\begin{aligned}
& \overline{\boldsymbol{f}}_{10}=-\boldsymbol{M}_{A} \overline{\boldsymbol{v}}_{f t}-\overline{\boldsymbol{\omega}}_{f} \times\left(\boldsymbol{M}_{A} \overline{\boldsymbol{v}}_{f}\right)-\boldsymbol{D}_{A 11} \overline{\boldsymbol{v}}_{f} \\
& \quad-\boldsymbol{D}_{A 12} \overline{\boldsymbol{v}}_{f} \otimes \overline{\boldsymbol{v}}_{f}+\overline{\boldsymbol{f}}_{10}^{\diamond}, \\
& \overline{\boldsymbol{f}}_{20}=-\boldsymbol{J}_{A} \overline{\boldsymbol{\omega}}_{f t}+\overline{\boldsymbol{\omega}}_{f} \times\left(\boldsymbol{J}_{A} \overline{\boldsymbol{\omega}}_{f}\right)+\overline{\boldsymbol{v}}_{f} \times\left(\boldsymbol{M}_{A} \overline{\boldsymbol{v}}_{\boldsymbol{f}}\right) \\
& \quad-\boldsymbol{D}_{A 21} \overline{\boldsymbol{\omega}}_{f}-\boldsymbol{D}_{A 22} \overline{\boldsymbol{\omega}}_{f} \otimes \overline{\boldsymbol{\omega}}_{f} \overline{\boldsymbol{\omega}}_{f}+\overline{\boldsymbol{f}}_{20}^{\diamond}, \\
& \boldsymbol{G}_{11}=-\boldsymbol{S}(\boldsymbol{\omega}) \boldsymbol{M}_{A}, \boldsymbol{G}_{12}=\boldsymbol{S}\left(\boldsymbol{M}_{A} \boldsymbol{v}\right), \boldsymbol{G}_{13}=3 \boldsymbol{D}_{A 12} \boldsymbol{v} \otimes \mathbf{1}, \\
& \boldsymbol{G}_{14}=6 \boldsymbol{D}_{A 12} \boldsymbol{v} \otimes \mathbf{1}, \boldsymbol{G}_{15}=-3 \boldsymbol{D}_{A 12} \boldsymbol{v} \otimes \boldsymbol{v}, \boldsymbol{G}_{16}=\boldsymbol{I}_{3}, \\
& \boldsymbol{G}_{21}=-\boldsymbol{S}(\boldsymbol{\omega}) \boldsymbol{J}_{A}, \boldsymbol{G}_{22}=\boldsymbol{S}\left(\boldsymbol{J}_{A} \boldsymbol{\omega}\right), \boldsymbol{G}_{23}=-\boldsymbol{S}(\boldsymbol{v}) \boldsymbol{M}_{A}, \\
& \boldsymbol{G}_{24}=\boldsymbol{S}\left(\boldsymbol{M}_{A} \boldsymbol{v}\right) \boldsymbol{G}_{25}=3 \boldsymbol{D}_{A 22} \boldsymbol{\omega} \otimes \mathbf{1}, \boldsymbol{G}_{26}=6 \boldsymbol{D}_{A 22} \boldsymbol{\omega} \otimes \mathbf{1}, \\
& \boldsymbol{G}_{27}=-3 \boldsymbol{D}_{A 22} \boldsymbol{\omega} \otimes \boldsymbol{\omega}, \boldsymbol{G}_{28}=\boldsymbol{I}_{3}, \mathbf{1}:=\operatorname{col}(1,1,1) .
\end{aligned}
$$

c) Contact forces and moments: The contact force and moment vectors $\boldsymbol{n}$ and $\boldsymbol{m}$ are:

$$
\begin{aligned}
& \boldsymbol{n}(s, t)=Q_{1}(s, t) \boldsymbol{b}_{1}+Q_{2}(s, t) \boldsymbol{b}_{2}+N(s, t) \boldsymbol{b}_{3}, \\
& \boldsymbol{m}(s, t)=M_{1}(s, t) \boldsymbol{b}_{1}+M_{2}(s, t) \boldsymbol{b}_{2}+T(s, t) \boldsymbol{b}_{3},
\end{aligned}
$$

where $Q_{1}$ and $Q_{2}$ are the shear forces; $N$ is the axial force; $M_{1}$ and $M_{2}$ are the bending moments; and $T$ is the twisting moment. Using the third-order Maclaurin series expansion of nonlinear stress-strain relations in [43] results in the constitutive equations:

$Q_{i}=G \bar{A}_{i}\left(\eta_{i}-\frac{1}{2} \eta_{i}^{2}+\frac{1}{3} \eta_{i}^{3}\right), N=E A\left(\varepsilon-\frac{1}{2} \varepsilon^{2}+\frac{1}{3} \varepsilon^{3}\right)+P_{0}$, $M_{i}=E I_{i}\left(\mu_{i}-\frac{1}{2} \mu_{i}^{2}+\frac{1}{3} \mu_{i}^{3}\right), T=G I_{3}\left(\mu_{3}-\frac{1}{2} \mu_{3}^{3}+\frac{1}{3} \mu_{3}^{3}\right)$

where $i=1,2 ; E$ is the Young modulus; $G$ is the shear modulus; $A, \bar{A}_{1}, \bar{A}_{2}$ are cross section and shear areas; $I_{k}, k=1,2,3$ are principal mass moments of inertia about $\boldsymbol{b}_{k}$; and $P_{0}$ is a positive constant axial force.

d) Initial conditions: The initial conditions are given by

$$
\begin{aligned}
& \tilde{\boldsymbol{r}}\left(s, t_{0}\right)=\tilde{\boldsymbol{r}}_{10}(s), \quad \tilde{\boldsymbol{r}}_{t}\left(s, t_{0}\right)=\tilde{\boldsymbol{r}}_{20}(s), \\
& \boldsymbol{\theta}\left(s, t_{0}\right)=\boldsymbol{\theta}_{0}(s), \quad \boldsymbol{\omega}\left(s, t_{0}\right)=\boldsymbol{\omega}_{0}(s) .
\end{aligned}
$$

e) Boundary conditions: Finally, referring to Fig. 1A the boundary conditions are

$$
\begin{aligned}
& \boldsymbol{\theta}(0, t)=0, \tilde{\boldsymbol{r}}(0, t)=0, \\
& d \tilde{\boldsymbol{r}}(\Gamma, t)=\tilde{\boldsymbol{r}}_{t}(\Gamma, t) d t, \quad d \boldsymbol{q}(\Gamma, t)=\boldsymbol{K}(\boldsymbol{q}(\Gamma, t)) \boldsymbol{\omega}(\Gamma, t) d t, \\
& d \tilde{\boldsymbol{r}}_{t}(\Gamma, t)=\boldsymbol{M}_{1 H}^{-1}\left[-\left(\boldsymbol{n}(\Gamma, t)-P_{0} \boldsymbol{e}_{3}\right)+\boldsymbol{f}_{1 B}+\boldsymbol{\phi}_{1 B}\right] d t \\
& +\boldsymbol{\Delta}_{1 B} d \boldsymbol{W}_{1 B} \\
& d \boldsymbol{\omega}(\Gamma, t)=\boldsymbol{M}_{2 H}^{-1}\left[-\boldsymbol{m}(\Gamma, t)+\boldsymbol{f}_{2 B}+\boldsymbol{\phi}_{2 B}\right] d t+\boldsymbol{\Delta}_{2 B} d \boldsymbol{W}_{2 B},
\end{aligned}
$$

where $\phi_{1 B}$ and $\phi_{2 B}$ are force and moment boundary control input vectors; $\boldsymbol{M}_{1 H}$ and $\boldsymbol{M}_{2 H}$ are mass and inertia matrices of the actuator systems; $\boldsymbol{\Delta}_{1 B}$ and $\boldsymbol{\Delta}_{2 B}$ are covariance matrices; $\boldsymbol{W}_{1 B}$ and $\boldsymbol{W}_{2 B}$ are vectors of standard Wiener processes; and $\boldsymbol{f}_{1 B}$ and $\boldsymbol{f}_{2 B}$ are given by

$$
\begin{aligned}
& \boldsymbol{f}_{1 B}=\boldsymbol{D}_{11 B} \tilde{\boldsymbol{r}}_{t}+\boldsymbol{f}_{1 B 0}+\boldsymbol{D}_{12 B}\left(\tilde{\boldsymbol{r}}_{t} \otimes \tilde{\boldsymbol{r}}_{t}\right) \tilde{\boldsymbol{r}}_{t}, \\
& \boldsymbol{f}_{2 B}=\boldsymbol{D}_{21 B} \boldsymbol{\omega}+\boldsymbol{f}_{2 B 0}+\boldsymbol{D}_{22 B}(\boldsymbol{\omega} \otimes \boldsymbol{\omega}) \boldsymbol{\omega},
\end{aligned}
$$

at $s=\Gamma$, where $\boldsymbol{D}_{i j B},(i, j)=1,2$ are negative definite matrices; $\boldsymbol{f}_{1 B 0}(t)$ and $\boldsymbol{f}_{2 B 0}(t)$ are bounded external disturbances acting on the actuators.

\section{Control objective}

Before stating the control objective, we make the following assumption, which is reasonable in practice.

\section{Assumption 3.1}

1) The initial values $\tilde{\boldsymbol{r}}_{10}(s), \tilde{\boldsymbol{r}}_{20}(s), \boldsymbol{\theta}_{0}(s), \boldsymbol{\omega}_{0}(s)$ are bounded in $L^{2}$-norm, i.e., $\exists \epsilon_{0} \geq 0$ such that $\int_{0}^{\Gamma}\left(\left\|\tilde{\boldsymbol{r}}_{10}(s)\right\|^{2}+\right.$ $\left.\left\|\tilde{\boldsymbol{r}}_{20}(s)\right\|^{2}+\left\|\boldsymbol{\theta}_{0}(s)\right\|^{2}+\left\|\boldsymbol{\omega}_{0}(s)\right\|^{2}\right) d s \leq \epsilon_{0}$. Moreover, $\left\|\tilde{\boldsymbol{r}}\left(\Gamma, t_{0}\right)\right\|^{2}+\left\|\tilde{\boldsymbol{r}}_{t}\left(\Gamma, t_{0}\right)\right\|^{2}+\left\|\boldsymbol{\theta}\left(\Gamma, t_{0}\right)\right\|^{2}+\left\|\boldsymbol{\omega}\left(\Gamma, t_{0}\right)\right\|^{2}$ is also bounded.

2) The damping matrices $\boldsymbol{D}_{\text {Aij }}(s, t),(i, j)=1,2$ are diagonal, negative definite, and bounded for all $(s, t) \in[0, \Gamma] \times\left[t_{0}, \infty\right)$, i.e., $\exists d_{A i j}^{m}>0, d_{A i j}^{M}>0$ such that $\int_{0}^{\Gamma} \boldsymbol{x}^{T} \boldsymbol{D}_{A i j} \boldsymbol{x} d s \leq-d_{A i j}^{m} \int_{0}^{\Gamma}\|\boldsymbol{x}\|^{2} d s$ and $\int_{0}^{\Gamma} \boldsymbol{y}^{T} \boldsymbol{D}_{A i j} \boldsymbol{x} d s \leq d_{A i j}^{M} \int_{0}^{\Gamma}\|\boldsymbol{x}\|\|\boldsymbol{y}\| d s$ for $\boldsymbol{x}, \boldsymbol{y} \in \mathbb{R}^{3}$ and $t \in[0, \infty)$. The damping matrices $\boldsymbol{D}_{i j B}(t),(i, j)=1,2$ are negative definite and bounded for all $t \in\left[t_{0}, \infty\right)$.

3) The deterministic components $\overline{\boldsymbol{v}}_{f}(s, t)$ and $\overline{\boldsymbol{\omega}}_{f}(s, t)$ are bounded for all $(s, t) \in[0, \Gamma] \times\left[t_{0}, \infty\right)$, i.e., $\exists \bar{v}_{f}^{M} \geq$ $0, \bar{\omega}_{f}^{M} \geq 0$ such that $\left\|\overline{\boldsymbol{v}}_{f}(s, t)\right\| \leq \bar{v}_{f}^{M}$ and $\left\|\overline{\boldsymbol{\omega}}_{f}(s, t)\right\| \leq$ $\bar{\omega}_{f}^{M}$. The deterministic components $\overline{\boldsymbol{f}}_{10}^{\diamond}(s, t)$ and $\overline{\boldsymbol{f}}_{20}^{\diamond}(s, t)$ of the external load vectors $\boldsymbol{f}_{10}^{\diamond}(s, t)$ and $\boldsymbol{f}_{20}^{\diamond}(s, t)$ are bounded in $L^{2}$-norm for all $t \in\left[t_{0}, \infty\right)$. The deterministic components $\boldsymbol{f}_{1 B 0}(t)$ and $\boldsymbol{f}_{2 B 0}(t)$ are bounded for all $t \in\left[t_{0}, \infty\right)$. The above boundedness implies that $\overline{\boldsymbol{f}}_{10}$ and $\overline{\boldsymbol{f}}_{20}$ are also bounded in $L^{2}$-norm for all $t \in\left[t_{0}, \infty\right)$.

4) All the covariances $\boldsymbol{\Delta}_{1 i}(s, t), i=1, \ldots, 6$ and $\boldsymbol{\Delta}_{2 i}(s, t), i=1, \ldots, 8$ are bounded in $L^{\infty}$-norm, i.e., $\exists \delta_{1 i}^{M} \geq 0$ and $\delta_{2 i}^{M} \geq 0$ such that $\int_{0}^{\Gamma}\left\|\boldsymbol{\Delta}_{1 i}(s, t)\right\|_{\infty} d s \leq \delta_{1 i}^{M}$ and $\int_{0}^{\Gamma}\left\|\boldsymbol{\Delta}_{2 i}(s, t)\right\|_{\infty} d s \leq \delta_{2 i}^{M}$. The covariances $\boldsymbol{\Delta}_{1 B}(t)$ and $\boldsymbol{\Delta}_{2 B}(t)$ are also bounded in norm infinity, i.e., $\exists \delta_{i B}^{M}>0, i=1,2$ such that $\left\|\boldsymbol{\Delta}_{i B}(t)\right\|_{\infty} \leq \delta_{i B}^{M}$. 
5) All the covariances and mean flows satisfy:

$$
\begin{aligned}
& d_{A 11}^{m}>\lambda_{M}\left(\left|\boldsymbol{M}_{A}\right|\right) \bar{\omega}_{f}^{M}+12 \lambda_{M}^{2}\left(\left|\boldsymbol{M}_{A}\right|\right)\left(\delta_{23}^{M}\right)^{2} \\
& \quad+12 \lambda_{M}^{2}\left(\left|\boldsymbol{M}_{A}\right|\right)\left(\delta_{24}^{M}\right)^{2}+9 \lambda_{M}^{2}\left(\left|\boldsymbol{M}_{A}\right|\right)\left(\delta_{12}^{M}\right)^{2} \\
& \quad+27 d_{A 12}\left(d_{A 12}^{M}\right)^{2}\left(\delta_{13}^{M}\right)^{2}+108 d_{A 12}\left(d_{A 12}^{M}\right)^{2}\left(\delta_{14}^{M}\right)^{2} \\
& \quad-3 d_{A 12}^{M} \bar{v}_{f}^{M} \epsilon_{01}-\lambda_{M}\left(\left|\boldsymbol{M}_{A}\right|\right) \bar{v}_{f}^{M} \epsilon_{03}, \\
& d_{A 12}^{m}>81\left(d_{A 12}^{M}\right)^{2}\left(\delta_{15}^{M}\right)^{2}-\frac{9 d_{A 12}^{M} \bar{v}_{f}^{M}}{4 \epsilon_{01}} \\
& \quad d_{A 21}^{m}>\lambda_{M}\left(\left|\boldsymbol{J}_{A}\right|\right) \bar{\omega}_{f}^{M}+3 d_{A 22}^{M}\left(\bar{\omega}_{f}^{M}\right)^{2}+9 \lambda_{M}^{2}\left(\left|\boldsymbol{M}_{A}\right|\right) \delta_{11}^{M} \\
& \quad+12 \lambda_{M}^{2}\left(\left|\boldsymbol{J}_{A}\right|\right)\left(\delta_{21}^{M}\right)^{2}+12 \lambda_{M}^{2}\left(\left|\boldsymbol{J}_{A}\right|\right)\left(\delta_{22}^{M}\right)^{2}+12\left(d_{A 22}^{M}\right)^{2} \\
& \quad \times\left(\delta_{25}^{M}\right)^{2}+24\left(d_{A 22}^{M}\right)^{2}\left(\delta_{26}^{M}\right)^{2}-\frac{\lambda_{M}\left(\left|\boldsymbol{M}_{A}\right|\right) \bar{v}_{f}^{M}}{4 \epsilon_{03}}-3 d_{A 21}^{M} \bar{\omega}_{f}^{M} \epsilon_{04}, \\
& d_{A 22}^{m}>36\left(d_{A 22}^{M}\right)^{2}\left(\delta_{27}^{M}\right)^{2}-\frac{9 d_{A 22}^{M} \bar{\omega}_{f}^{M}}{4 \epsilon_{04}}
\end{aligned}
$$

where $\epsilon_{0 i}, i=1,3,4$ are positive constants. The above inequalities imply that all the covariances and mean flows are not too large in comparison with the damping coefficients. We require the condition (19) because of the stochastic component of fluid velocities affine with the beam's velocities, see (8).

6) The beam is relatively slender, i.e., the shear magnitude is smaller than that of the gradient of the transverse displacements:

$$
\eta_{1}^{2}(s, t)+\eta_{2}^{2}(s, t) \leq \tilde{x}_{s}^{2}(s, t)+\tilde{y}_{s}^{2}(s, t),
$$

for all $(s, t) \in[0, \Gamma] \times\left[t_{0}, \infty\right)$.

Control Objective 3.1 Under Assumption 3.1, design the positive constant axial force $P_{0}$ and the boundary control vectors $\phi_{i B}, i=1,2$ such that the beam system consisting of (11)-(17) is globally (practically) $\mathcal{K}_{\infty}^{-}$ exponentially stable at the origin in the sense that

$$
\mathbb{E} \mathcal{E}(t) \leq \alpha\left(\mathcal{E}\left(t_{0}\right)\right) e^{-c\left(t-t_{0}\right)}+c_{0},
$$

where $\alpha$ is a class $\mathcal{K}_{\infty}$-function, $c$ is a positive constant, and the constant $c_{0}$ is zero if $\overline{\boldsymbol{f}}_{i 0}^{\diamond}(s, t)=0, \boldsymbol{f}_{i B 0}(t)=$ $0, i=1,2$ for all $t \in\left[t_{0}, \infty\right)$ and $s \in[0, \Gamma]$ and all the stochastic terms are zero, and is a positive constant if the above terms satisfy Assumption 3.1, and

$$
\begin{aligned}
\mathcal{E}(t) & =\int_{0}^{\Gamma}\left[\|\boldsymbol{v}(s, t)\|^{2}+\|\boldsymbol{\omega}(s, t)\|^{2}+\|\boldsymbol{\vartheta}(s, t)\|^{2}+\|\boldsymbol{\mu}(s, t)\|^{2}\right. \\
& +\left(\eta_{1}^{4}(s, t)+\eta_{2}^{4}(s, t)+\varepsilon^{4}(s, t)\right)+\left(\mu_{1}^{4}(s, t)+\mu_{2}^{4}(s, t)\right. \\
& \left.\left.+\mu_{3}^{4}(s, t)\right)\right] d s+\left\|\gamma \Gamma \tilde{\boldsymbol{r}}_{s}(\Gamma, t)+\tilde{\boldsymbol{r}}_{t}(\Gamma, t)\right\|^{2} \\
& +\|\gamma \Gamma \boldsymbol{\mu}(\Gamma, t)+\boldsymbol{\omega}(\Gamma, t)\|^{2},
\end{aligned}
$$

where

$$
\boldsymbol{\vartheta}(s, t):=\operatorname{col}\left(\eta_{1}(s, t), \eta_{2}(s, t), \varepsilon(s, t)\right),
$$

and $\gamma$ is a positive constant (to be chosen later). Note that $\mathcal{E}(t)$ is a positive definite and radially unbounded functional of velocities, stretch, shear strain, bending and torsional curvatures. Several useful equalities and inequalities are given in the following lemma.
Lemma 3.1 For all $t \geq t_{0} \geq 0$ and $s \in[0, \Gamma]$, we have:

$$
\begin{aligned}
& \varepsilon-\tilde{z}_{s}=-\frac{1}{2}\|\boldsymbol{\vartheta}\|^{2}+\frac{1}{2}\|\boldsymbol{\mu}\|^{2}, \varepsilon-\tilde{z}_{s} \geq 0, \\
& \boldsymbol{\omega}_{s}=\mu_{1 t} \boldsymbol{b}_{1}+\mu_{2 t} \boldsymbol{b}_{2}+\mu_{3 t} \boldsymbol{b}_{3}, \\
& \boldsymbol{\omega}^{T}\left(\boldsymbol{r}_{s} \times \boldsymbol{n}\right)=\boldsymbol{n}^{T}\left(\eta_{1} \boldsymbol{b}_{1 t}+\eta_{2} \boldsymbol{b}_{2 t}+(1+\varepsilon) \boldsymbol{b}_{3 t}\right), \\
& \boldsymbol{\mu}^{T}\left(\boldsymbol{r}_{s} \times \boldsymbol{n}\right)=-\boldsymbol{n}^{T}\left(\eta_{1} \boldsymbol{b}_{1 s}+\eta_{2} \boldsymbol{b}_{2 s}+(1+\varepsilon) \boldsymbol{b}_{3 s}\right), \\
& \boldsymbol{n}^{T} \tilde{\boldsymbol{r}}_{s t}=Q_{1} \eta_{1 t}+Q_{2} \eta_{2 t}+N \varepsilon_{t}+\boldsymbol{\omega}^{T}\left(\boldsymbol{r}_{s} \times n\right), \\
& \boldsymbol{n}^{T}\left(\boldsymbol{r}_{s}+\boldsymbol{r}_{s s} s\right)=Q_{1} \eta_{1}+Q_{2} \eta_{2}+N(1+\varepsilon)+Q_{1} \eta_{1 s} s \\
& \quad+Q_{2} \eta_{2 s} s+N \varepsilon_{s} s+\boldsymbol{n}^{T}\left(\boldsymbol{\mu} \times \boldsymbol{r}_{s}\right) s, \\
& \left\|\tilde{\boldsymbol{r}}_{s}\right\|_{L^{2}}^{2} \leq 2\|\boldsymbol{\vartheta}\|_{L^{2}}^{2}+384 \Gamma^{2}\|\boldsymbol{\mu}\|_{L^{2}}^{2},
\end{aligned}
$$

where we have dropped the argument $(s, t)$ for clarity.

Proof. The last inequality is proved by using (2), (3), and (4). For proof of all others, see Lemma 3.1 in [9].

\section{Well-posedness and Stability of SESs}

\subsection{Space notations}

The notations in [47] will be used in study of wellposedness and stability of SESs in the sequel. Let $\left(V, H, V^{*}\right)$ be a Gelfand triple, i.e., $\left(H,\langle., .\rangle_{H}\right)$ is a separable Hilbert space identified with its dual $H^{*}$ via the Riesz isomorphism, $V$ is a reflexible Banach space embedded in $H$ continuously and densely. Denote by $\langle., .\rangle_{V, V^{*}}$ the dual product between $V$ and its dual $V^{*}$. Let $\boldsymbol{W}(t)$ be a $\boldsymbol{Q}$-Wiener process defined on another separable Hilbert space with respect to a probability space $(\Omega, \mathcal{F}, \mathbb{P})$. Let $\|\cdot\|_{H},\|\cdot\|_{V}$, and $\|\cdot\|_{V^{*}}$ denote the norms in $H, V$, and $V^{*}$. Denote by $\mathscr{L}_{2}\left(\boldsymbol{Q}^{\frac{1}{2}}, H\right)$ the Hilbert space of all Hilbert-Schmidt operators from $\boldsymbol{Q}^{\frac{1}{2}}$ to $H$ equipped with the norm $\|\mathbf{\Phi}\|_{\mathscr{L}_{2}^{0}}^{2}=\operatorname{Tr}\left(\boldsymbol{\Phi} \boldsymbol{Q} \boldsymbol{\Phi}^{\star}\right)$ for $\boldsymbol{\Phi} \in \mathscr{L}_{2}^{0}$, where Tr stands for the trace operator and $\star$ is the adjoint operation, and $\mathscr{L}(K, H)$ the set of all linear bounded operators from $K$ into $H$.

\subsection{Stochastic evolution systems}

Consider the following nonlinear SES on the space $H$ :

$$
\begin{aligned}
& d \boldsymbol{X}(t)=\boldsymbol{F}(\boldsymbol{X}(t), t) d t+\boldsymbol{G}(\boldsymbol{X}(t), t) d \boldsymbol{W}(t), \\
& \boldsymbol{X}\left(t_{0}\right)=\boldsymbol{X}_{0} \in V,
\end{aligned}
$$

where $\boldsymbol{F}: H \times\left[t_{0}, \infty\right) \rightarrow V^{*}$ and $\boldsymbol{G}: H \times\left[t_{0}, \infty\right) \rightarrow$ $\mathscr{L}_{2}\left(K_{0}, H\right)$ are Borel measurable. We first define the variational solution of (25) as in [7].

Definition 4.1 An $\mathcal{F}_{t}$-measurable $H$-valued stochastic process $\left\{\boldsymbol{X}(t), t \in\left[t_{0}, \infty\right)\right\}$ is said to be a global variational strong solution of (25) if for any $\boldsymbol{\psi} \in V$ we have for each $t \in\left[t_{0}, \infty\right)$

$$
\begin{aligned}
\langle\boldsymbol{\psi}, \boldsymbol{X}(t)\rangle_{H}= & \left\langle\boldsymbol{\psi}, \boldsymbol{X}_{0}\right\rangle_{H}+\int_{t_{0}}^{t}\langle\boldsymbol{\psi}, \boldsymbol{F}(\boldsymbol{X}(s), s)\rangle_{V, V^{*}} d s \\
& +\int_{t_{0}}^{t}\langle\boldsymbol{\psi}, \boldsymbol{G}(\boldsymbol{X}(s), s) d \boldsymbol{W}(s)\rangle_{H} .
\end{aligned}
$$

In (26), the time integral is in Itô's sense while the spatial integrals $\langle., .\rangle_{H}$ and $\langle., .\rangle_{V, V^{*}}$ are in the weak sense. Thus, the solution $\boldsymbol{X}(t)$ is strong in the stochastic sense but weak (variational) in the PDE theory. We give a definition of stability of the system (25) based on $[19,45,46]$. 
Definition 4.2 The system (25) is said to be globally practically $\mathcal{K}_{\infty}$-exponentially $p$-stable $(p \geq 2)$ at the origin if its variational strong solution satisfies

$$
\mathbb{E}\left\{\|\boldsymbol{X}(t)\|_{H}^{p}\right\} \leq \alpha\left(\left\|\boldsymbol{X}_{0}\right\|_{V}^{p}\right) e^{-\epsilon\left(t-t_{0}\right)}+\epsilon_{0},
$$

for all $\boldsymbol{X}_{0} \in V$ with $\left\|\boldsymbol{X}_{0}\right\|_{V}^{p}<\infty$ and $t \in\left[t_{0}, \infty\right)$, where $\alpha$ is a class $\mathcal{K}_{\infty}$-function, and $\epsilon_{0}$ and $\epsilon$ are positive constants. If $\epsilon_{0}=0$, then it is said to be globally $\mathcal{K}_{\infty}$-exponentially p-stable at the origin. If $\alpha\left(\left\|\boldsymbol{X}_{0}\right\|_{V}^{p}\right) \equiv$ $\epsilon_{1}\left\|\boldsymbol{X}_{0}\right\|_{V}^{p}$, where $\epsilon_{1}$ is a positive constant, then "K $\mathcal{K}_{\infty}$ " is dropped in the above definition.

\subsection{The Itô formula}

Lemma 4.1 Let $C^{2}\left(H \times\left[t_{0}, \infty\right)\right)$ be the space of all realvalued nonnegative functions $U(\boldsymbol{X}, t)$ on $H \times\left[t_{0}, \infty\right)$ such that:

1) $U(\boldsymbol{X}, t)$ is twice (Fréchet) differentiable in $\boldsymbol{X}$ and is differentiable in $t$.

2) Both $U_{\boldsymbol{X}}(\boldsymbol{X}, t)$ and $U_{t}(\boldsymbol{X}, t)$ are continuous in $H \times\left[t_{0}, \infty\right)$ and $U_{\boldsymbol{X} \boldsymbol{X}}(\boldsymbol{X}, t)$ is continuous in $\mathscr{L}(H, H) \times$ $\left[t_{0}, \infty\right)$.

Suppose that $\boldsymbol{X}(t)$ is a solution of (25). Then [46]:

$$
\begin{aligned}
U(\boldsymbol{X}, t) & =U\left(\boldsymbol{X}_{0}, t_{0}\right)+\int_{t_{0}}^{t} \mathcal{L} U(\boldsymbol{X}(s), s) d s \\
& +\int_{t_{0}}^{t}\left\langle U_{\boldsymbol{X}}(\boldsymbol{X}(s), s), \boldsymbol{G}(\boldsymbol{X}(s), s) d \boldsymbol{W}(s)\right\rangle_{H},
\end{aligned}
$$

where the infinitesimal generator $\mathcal{L} U(\boldsymbol{X}, t)$ is given by

$$
\begin{gathered}
\mathcal{L} U(\boldsymbol{X}, t)=U_{t}(\boldsymbol{X}, t)+\left\langle U_{\boldsymbol{X}}(\boldsymbol{X}, t), \boldsymbol{F}(\boldsymbol{X}, t)\right\rangle_{V, V^{*}} \\
+\frac{1}{2} \operatorname{Tr}\left(U_{\boldsymbol{X} \boldsymbol{X}}(\boldsymbol{X}, t) \boldsymbol{G}(\boldsymbol{X}, t) \boldsymbol{Q} \boldsymbol{G}^{\star}(\boldsymbol{X}, t)\right) .
\end{gathered}
$$

Assumption 4.1 The following conditions are imposed on $\boldsymbol{F}$ and $\boldsymbol{G}$ to prove local well-posedness of (25):

H0. There exists an orthogonal set $\left\{e_{1}, e_{2}, \ldots\right\}$ in $V$ such that it constitutes an orthogonal basis of $H$.

H1. [Continuity] The following mappings are continuous:

$V \times\left[t_{0}, \infty\right) \ni(\boldsymbol{v}, t) \mapsto \boldsymbol{F}(\boldsymbol{v}, t) \in V^{*}$,

$V \times\left[t_{0}, \infty\right) \ni(\boldsymbol{v}, t) \mapsto\left(\boldsymbol{G}(\boldsymbol{v}, t) \boldsymbol{Q}^{\frac{1}{2}}\right)\left(\boldsymbol{G}(\boldsymbol{v}, t) \boldsymbol{Q}^{\frac{1}{2}}\right)^{*} \in \mathscr{L}(H, H)$.

H2. [Local monotonicity] For any $\boldsymbol{u}, \boldsymbol{v} \in V$ with $\|\boldsymbol{u}\|_{V} \vee$ $\|\boldsymbol{v}\|_{V} \leq \varepsilon$, there exists a constant $c_{\epsilon}$ such that

$$
\begin{gathered}
2\langle\boldsymbol{u}-\boldsymbol{v}, \boldsymbol{F}(\boldsymbol{u}, t)-\boldsymbol{F}(\boldsymbol{v}, t)\rangle_{V, V^{*}}+\|\boldsymbol{G}(\boldsymbol{u}, t)-\boldsymbol{G}(\boldsymbol{v}, t)\|_{\mathscr{L}_{2}^{0}}^{2} \\
\leq c_{\epsilon}\|\boldsymbol{u}-\boldsymbol{v}\|_{H}^{2} .
\end{gathered}
$$

H3. [Local growth] There exists a constant $\delta$ such that:

$$
\|\boldsymbol{F}(\boldsymbol{u}, t)\|_{V^{*}} \leq \delta\left(1+\|\boldsymbol{u}\|_{V}^{p-1}\right) \forall \boldsymbol{u} \in V,\|\boldsymbol{u}\|_{V} \leq \epsilon .
$$

Theorem 4.1 Under Assumption 4.1, assume further that there exists a function $U \in C^{2}\left(H \times\left[t_{0}, \infty\right)\right)$ called a Lyapunov function; and class $\mathcal{K}_{\infty}$-functions $\alpha_{1}$ and $\alpha_{2}$ such that $\forall(\boldsymbol{X}, t) \in V \times\left[t_{0}, \infty\right)$ :

$$
\alpha_{1}\left(\|\boldsymbol{X}\|_{V}^{p}\right) \leq U(\boldsymbol{X}, t) \leq \alpha_{2}\left(\|\boldsymbol{X}\|_{V}^{p}\right)
$$

1) [Well-posedness] Suppose that $\mathcal{L} U(\boldsymbol{X}, t)$ satisfies

$$
\mathcal{L} U(\boldsymbol{X}, t) \leq c_{3}(1+U(\boldsymbol{X}, t)), \quad \boldsymbol{X} \in V, t \in\left[t_{0}, \infty\right),
$$

where $c_{3}$ is a nonnegative constant. Then the system (25) has a unique variational solution for each $\boldsymbol{X}_{0} \in V$.

2) [Stability] Suppose that there exists a positive constant $c_{4}$ such that $\forall(\boldsymbol{X}, t) \in V \times\left[t_{0}, \infty\right)$ :

$$
\mathcal{L} U(\boldsymbol{X}, t) \leq-c_{4} U(\boldsymbol{X}, t)+c_{0},
$$

if $c_{0}=0$, the system (25) is globally $p$-stable and globally $\mathcal{K}_{\infty}$-exponentially $p$-stable at the origin. If $c_{0}>0$, the system (25) is globally p-stable and globally practically $\mathcal{K}_{\infty}$-exponentially p-stable at the origin. Moreover:

$$
\mathbb{E}\left\{\|\boldsymbol{X}(t)\|_{V}^{p}\right\} \leq \alpha_{1}^{-1}\left(\alpha_{2}\left(\left\|\boldsymbol{X}\left(t_{0}\right)\right\|_{V}^{p}\right) e^{-c_{4}\left(t-t_{0}\right)}+\frac{c_{0}}{c_{4}}\right),
$$

where $\alpha_{1}^{-1}$ is the inverse function of $\alpha_{1}$, and $\circ$ denotes the composition operator. If $\alpha_{1}\left(\|\boldsymbol{X}\|_{V}^{p}\right) \equiv c_{1}\|\boldsymbol{X}\|_{V}^{p}$ and $\alpha_{2}\left(\|\boldsymbol{X}\|_{V}^{p}\right) \equiv c_{2}\|\boldsymbol{X}\|_{V}^{p}$ with $c_{1}$ and $c_{2}$ being positive constants, then "K $\mathcal{K}_{\infty}$ " is dropped in the above statement.

Proof. See Appendix A.

\section{Control design}

\subsection{Abstract formulation}

The modern approach to study a stochastic PDE is to regard its solution as a function of $t$ with values in a space of functions of $s$, see $[10,19]$. This approach is applied to our present beam problem in what follows. Let $L^{2}(\mathcal{D})$ denote the $L^{2}$-space with the norm $\|\cdot\|_{L^{2}}$ and inner product $\langle., .\rangle_{L^{2}}$ and $W^{m, p}(\mathcal{D})$, with $(m, p)$ being integers, denote the Sobolev space of order $m$ and degree $p$, see [1]. Considering $s \in[0, \Gamma]$ as the parameter defined at every $t \geq t_{0}$, we can regard $\tilde{\boldsymbol{r}}(s, t), \boldsymbol{q}(s, t), \boldsymbol{v}(s, t)$, and $\boldsymbol{\omega}(s, t)$ as $\overline{\tilde{\boldsymbol{r}}}(t) \in\left(W^{2,2}([0, \Gamma])\right)^{3}, \boldsymbol{q}(t) \in\left(W^{2,2}([0, \Gamma])\right)^{4}$, $\boldsymbol{v}(t) \in\left(L^{2}([0, \Gamma])\right)^{3}, \boldsymbol{\omega}(t) \in\left(L^{2}([0, \Gamma])\right)^{3}$, respectively. Similarly, $\tilde{\boldsymbol{r}}(0, t), \boldsymbol{q}(0, t), \boldsymbol{v}(0, t)$, and $\boldsymbol{\omega}(0, t)$ are regarded as $\tilde{\boldsymbol{r}}^{B 0}(t) \in\left(W^{2,2}([0, \Gamma])\right)^{3}, \boldsymbol{q}^{B 0}(t) \in\left(W^{2,2}([0, \Gamma])\right)^{4}$, $\boldsymbol{v}^{B 0}(t) \in\left(L^{2}([0, \Gamma])\right)^{3}, \boldsymbol{\omega}^{B 0}(t) \in\left(L^{2}([0, \Gamma])\right)^{3}$, respectively. Moreover, $\tilde{\boldsymbol{r}}(\Gamma, t), \boldsymbol{q}(\Gamma, t), \boldsymbol{v}(\Gamma, t)$, and $\boldsymbol{\omega}(\Gamma, t)$ are considered as $\tilde{\boldsymbol{r}}^{B \Gamma}(t) \in\left(W^{2,2}(\Gamma)\right)^{3}, \boldsymbol{q}^{B \Gamma}(t) \in$ $\left(W^{2,2}(\Gamma)\right)^{4}, \boldsymbol{v}^{B \Gamma}(t) \in\left(L^{2}(\Gamma)\right)^{3}, \boldsymbol{\omega}^{B \Gamma}(t) \in\left(L^{2}(\Gamma)\right)^{3}$, respectively. Let us also define $\mathbb{D} \phi(s):=\frac{\partial \phi}{\partial s}$. Thus, we can write the beam system (11) in the SES (abstract form):

$$
\begin{aligned}
d \tilde{\boldsymbol{r}}= & \boldsymbol{R}_{1}(\boldsymbol{q}) \boldsymbol{v} d t, \quad d \boldsymbol{q}=\boldsymbol{K}(\boldsymbol{q}) \boldsymbol{\omega} d t, \\
d \boldsymbol{v}= & \boldsymbol{M}^{-1}\left[-\boldsymbol{\omega} \times(\boldsymbol{M} \boldsymbol{v})+\boldsymbol{R}_{1}^{-1}(\boldsymbol{q}) \mathbb{D} \boldsymbol{n}+\boldsymbol{D}_{A 11} \boldsymbol{v}\right. \\
& \left.+\boldsymbol{D}_{A 12} \boldsymbol{v} \otimes \boldsymbol{v} \boldsymbol{v}+\boldsymbol{f}_{1}\right] d t+\boldsymbol{M}^{-1} \boldsymbol{G}_{1}(\boldsymbol{v}, \boldsymbol{\omega}) \boldsymbol{\Delta}_{1} d \boldsymbol{W}_{1} \\
& :=\overline{\boldsymbol{F}}_{1} d t+\overline{\boldsymbol{G}}_{1} d \boldsymbol{W}_{1}, \\
d \boldsymbol{\omega}= & \boldsymbol{J}^{-1}\left[\mathbb{D} \boldsymbol{m}-\boldsymbol{\omega} \times(\boldsymbol{J} \boldsymbol{\omega})+\boldsymbol{r}_{s} \times \boldsymbol{n}+\boldsymbol{v} \times\left(\boldsymbol{M}_{A} \boldsymbol{v}\right)\right. \\
& \left.+\boldsymbol{D}_{A 21} \boldsymbol{\omega}+\boldsymbol{D}_{A 22} \boldsymbol{\omega} \otimes \boldsymbol{\omega} \boldsymbol{\omega}+\boldsymbol{f}_{2}\right] d t+\boldsymbol{G}_{2}(\boldsymbol{v}, \boldsymbol{\omega}) \boldsymbol{\Delta}_{2} d \boldsymbol{W}_{2}, \\
& :=\overline{\boldsymbol{F}}_{2} d t+\overline{\boldsymbol{G}}_{2} d \boldsymbol{W}_{2},
\end{aligned}
$$

where the argument $t$ was dropped for clarity. The initial conditions (16) are written in the abstract form as:

$$
\tilde{\boldsymbol{r}}_{1}\left(t_{0}\right)=\tilde{\boldsymbol{r}}_{10}, \tilde{\boldsymbol{r}}_{2}\left(t_{0}\right)=\tilde{\boldsymbol{r}}_{20}, \boldsymbol{\theta}\left(t_{0}\right)=\boldsymbol{\theta}_{0}, \boldsymbol{\omega}\left(t_{0}\right)=\boldsymbol{\omega}_{0} .
$$


The boundary conditions (17) are written as:

$$
\begin{aligned}
& \boldsymbol{m}^{B 0}=0, \boldsymbol{v}^{B 0}=0, \\
& d \tilde{\boldsymbol{r}}^{B \Gamma}=\tilde{\boldsymbol{r}}_{t}^{B \Gamma} d t, \quad d \boldsymbol{q}^{B \Gamma}=\boldsymbol{K}\left(\boldsymbol{q}^{B \Gamma}\right) \boldsymbol{\omega}^{B \Gamma} d t, \\
& d \tilde{\boldsymbol{r}}_{t}^{B \Gamma}=\boldsymbol{M}_{1 H}^{-1}\left[-\left(\boldsymbol{n}^{B \Gamma}-P_{0} \boldsymbol{e}_{3}\right)+\boldsymbol{f}_{1 B}+\boldsymbol{\phi}_{1 B}\right] d t \\
& \quad+\boldsymbol{\Delta}_{1 B} d \boldsymbol{W}_{1 B}:=\overline{\boldsymbol{F}}_{1 B} d t+\overline{\boldsymbol{G}}_{1 B} d \boldsymbol{W}_{2 B}, \\
& d \boldsymbol{\omega}^{B \Gamma}=\boldsymbol{M}_{2 H}^{-1}\left[-\boldsymbol{m}^{B \Gamma}+\boldsymbol{f}_{2 B}+\boldsymbol{\phi}_{2 B}\right] d t \\
& \quad+\boldsymbol{\Delta}_{2 B} d \boldsymbol{W}_{2 B}=\overline{\boldsymbol{F}}_{2 B} d t+\overline{\boldsymbol{G}}_{2 B} d \boldsymbol{W}_{2 B},
\end{aligned}
$$

where $\boldsymbol{m}^{B 0}$ is the value of $\boldsymbol{m}$ evaluated at $s=0 ; \boldsymbol{n}^{B \Gamma}$ and $\boldsymbol{m}^{B \Gamma}$ are the values of $\boldsymbol{n}$ and $\boldsymbol{m}$ evaluated at $s=\Gamma$.

\subsection{Control design}

To design the boundary control vectors $\phi_{i B}, i=1,2$, we consider the following Lyapunov functional candidate:

$$
U=U_{0}+U_{1}+U_{2}
$$

where the functionals $U_{0}, U_{1}$, and $U_{2}$ are defined in the sequel. The functional $U_{0}$ is chosen as follows:

$$
U_{0}=U_{01}+U_{02},
$$

where

$$
\begin{aligned}
& U_{01}=\frac{1}{2}\langle\boldsymbol{v}, \boldsymbol{M} \boldsymbol{v}\rangle_{L^{2}}^{2}+\frac{1}{2}\langle\boldsymbol{\omega}, \boldsymbol{J} \boldsymbol{\omega}\rangle_{L^{2}}, \\
& U_{02}=\sum_{i=1}^{2} G \bar{A}_{i}\left\langle\eta_{i},\left(\frac{1}{2} \eta_{i}-\frac{1}{6} \eta_{i}^{2}+\frac{1}{12} \eta_{i}^{3}\right)\right\rangle_{L^{2}} \\
& \quad+E A\left\langle\varepsilon,\left(\frac{1}{2} \varepsilon-\frac{1}{6} \varepsilon^{2}+\frac{1}{12} \varepsilon^{3}\right)\right\rangle_{L^{2}} \\
& \quad+\sum_{i=1}^{2} E I_{i}\left\langle\mu_{i},\left(\frac{1}{2} \mu_{i}-\frac{1}{6} \mu_{i}^{2}+\frac{1}{12} \mu_{i}^{3}\right)\right\rangle_{L^{2}} \\
& +G I_{3}\left\langle\mu_{3},\left(\frac{1}{2} \mu_{3}-\frac{1}{6} \mu_{3}^{2}+\frac{1}{12} \mu_{3}^{3}\right)\right\rangle_{L^{2}}+P_{0}\langle 1, \varepsilon-\mathbb{D} \tilde{z}\rangle_{L^{2}} .
\end{aligned}
$$

The functional $U_{1}$ is chosen as follows

$$
U_{1}=U_{11}+U_{12},
$$

where

$$
U_{11}=\gamma\left\langle\mathbb{D} \tilde{\boldsymbol{r}}, \boldsymbol{R}_{1}(\boldsymbol{q}) \boldsymbol{M} \boldsymbol{v} s\right\rangle_{L^{2}}, U_{12}=\gamma\langle\boldsymbol{\mu}, \mathbb{D}(\boldsymbol{J} \boldsymbol{\omega}) s\rangle_{L^{2}},
$$

with $\gamma$ being a positive constant to be chosen later. The functional $U_{2}$ is chosen as follows

$$
\begin{gathered}
U_{2}=\frac{1}{2}\left(\gamma \Gamma \mathbb{D} \tilde{\boldsymbol{r}}^{B \Gamma}+\tilde{\boldsymbol{r}}_{t}^{B \Gamma}\right)^{T} \boldsymbol{M}_{1 H}\left(\gamma \Gamma \mathbb{D} \tilde{\boldsymbol{r}}^{B \Gamma}+\tilde{\boldsymbol{r}}_{t}^{B \Gamma}\right) \\
+\frac{1}{2}\left(\gamma \Gamma \boldsymbol{\mu}^{B \Gamma}+\boldsymbol{\omega}^{B \Gamma}\right)^{T} \boldsymbol{M}_{2 H}\left(\gamma \Gamma \boldsymbol{\mu}^{B \Gamma}+\boldsymbol{\omega}^{B \Gamma}\right) .
\end{gathered}
$$

We now find the bounds of $U$. Using the last inequality of $(24)$ and $\frac{1}{3} x^{2}+\frac{1}{12} x^{4} \leq \frac{1}{2}\left(x^{2}-\frac{1}{3} x^{3}+\frac{1}{4} x^{4}\right) \leq \frac{2}{3} x^{2}+\frac{1}{6} x^{4}$ for all $x \in \mathbb{R}$, we can bound $U_{02}$ and $U_{1}$ :

$$
\begin{aligned}
& U_{02} \leq\left(G \bar{A}_{1} \vee G \bar{A}_{2} \vee E A\right)\left(\frac{2}{3}\|\boldsymbol{\vartheta}\|_{L^{2}}^{2}+\frac{1}{6}\left(\left\|\eta_{1}^{2}\right\|_{L^{2}}^{2}+\left\|\eta_{2}^{2}\right\|_{L^{2}}^{2}\right.\right. \\
& \left.+\left\|\varepsilon^{2}\right\|_{L^{2}}^{2}\right)+\left(E I_{1} \vee E I_{2} \vee G I_{3}\right)\left(\frac{2}{3}\|\boldsymbol{\mu}\|_{L^{2}}^{2}+\frac{1}{6}\left(\left\|\mu_{1}^{2}\right\|_{L^{2}}^{2}+\left\|\mu_{2}^{2}\right\|_{L^{2}}^{2}\right.\right. \\
& \left.+\left\|\mu_{3}^{2}\right\|_{L^{2}}^{2}\right)+\frac{3}{2} P_{0}\|\boldsymbol{\vartheta}\|_{L^{2}}+384 \Gamma^{2} P_{0}\|\boldsymbol{\mu}\|_{L^{2}}^{2} \\
& U_{02} \geq\left(G \bar{A}_{1} \wedge G \bar{A}_{2} \wedge E A\right)\left(\frac{1}{3}\|\boldsymbol{\vartheta}\|_{L^{2}}^{2}+\frac{1}{12}\left(\left\|\eta_{1}^{2}\right\|_{L^{2}}^{2}+\left\|\eta_{2}^{2}\right\|_{L^{2}}^{2}\right.\right. \\
& \left.+\left\|\varepsilon^{2}\right\|_{L^{2}}^{2}\right)+\left(E I_{1} \wedge E I_{2} \wedge G I_{3}\right)\left(\frac{1}{3}\|\boldsymbol{\mu}\|_{L^{2}}^{2}+\frac{1}{12}\left(\left\|\mu_{1}^{2}\right\|_{L^{2}}^{2}+\left\|\mu_{2}^{2}\right\|_{L^{2}}^{2}\right.\right. \\
& \left.+\left\|\mu_{3}^{2}\right\|_{L^{2}}^{2}\right), \\
& \left|U_{1}\right| \leq \gamma \Gamma \lambda_{M}(\boldsymbol{M}) \varrho_{01}\|\boldsymbol{v}\|_{L^{2}}^{2}+\frac{\gamma \Gamma \lambda_{M}(\boldsymbol{M})}{4 \varrho_{01}}\left(2\|\boldsymbol{\vartheta}\|_{L^{2}}^{2}\right. \\
& \left.\quad+384 \Gamma^{2}\|\boldsymbol{\mu}\|_{L^{2}}^{2}\right)+\gamma \Gamma \lambda_{M}(\boldsymbol{J}) \varrho_{02}\|\boldsymbol{\omega}\|_{L^{2}}^{2}+\frac{\gamma \Gamma \lambda_{M}(\boldsymbol{J})}{4 \varrho_{02}}\|\boldsymbol{\mu}\|_{L^{2}}^{2},
\end{aligned}
$$

where $\varrho_{01}$ and $\varrho_{02}$ are positive constants to be determined; $\lambda_{M}(\bullet)$ denotes the maximum eigenvalue of $\bullet$; and $\boldsymbol{\vartheta}$ is defined in (23). Using (46), we can bound $U$ as:

$$
c_{1} \mathcal{E} \leq U \leq c_{2} \mathcal{E},
$$

where $\mathcal{E}$ is given in (22), and $c_{1}$ and $c_{2}$ are:

$$
\begin{aligned}
c_{1}= & {\left[\frac{\lambda_{m}(\boldsymbol{M})}{2}-\gamma \Gamma \varrho_{01} \lambda_{M}(\boldsymbol{M})\right] \wedge\left[\frac{\lambda_{m}(\boldsymbol{J})}{2}-\gamma \Gamma \varrho_{02} \lambda_{M}(\boldsymbol{J})\right] } \\
& \wedge\left[\frac{1}{3}\left(E A \wedge G \bar{A}_{1} \wedge G \bar{A}_{2}\right)-\frac{\gamma \Gamma \lambda_{M}(\boldsymbol{M})}{2 \varrho_{01}}\right] \wedge\left[\frac{\lambda_{m}\left(\boldsymbol{M}_{1 H}\right)}{2}\right] \\
& \wedge\left[\frac{1}{3}\left(E I_{1} \wedge E I_{2} \wedge G I_{3}\right)-\frac{\gamma \Gamma \lambda_{M}(\boldsymbol{J})}{4 \varrho_{02}}-\frac{96 \gamma \Gamma^{3} \lambda_{M}(\boldsymbol{M})}{\varrho_{01}}\right] \\
& \wedge\left[\frac{\lambda_{m}\left(\boldsymbol{M}_{2 H}\right)}{2}\right] \wedge \frac{1}{12}\left[E A \wedge G \bar{A}_{1} \wedge G \bar{A}_{2}\right] \\
& \wedge \frac{1}{12}\left[E I_{1} \wedge E I_{2} \wedge G I_{3}\right], \\
c_{2}= & {\left[\frac{\lambda_{M}(\boldsymbol{M})}{2}+\gamma \Gamma \varrho_{01} \lambda_{M}(\boldsymbol{M})\right] \vee\left[\frac{\lambda_{M}(\boldsymbol{J})}{2}+\gamma \Gamma \varrho_{02} \lambda_{M}(\boldsymbol{J})\right] } \\
& \vee\left[\frac{2}{3}\left(E A \vee G \bar{A}_{1} \vee G \bar{A}_{2}\right)+\frac{\gamma \Gamma \lambda_{M}(\boldsymbol{M})}{2 \varrho_{01}}+\frac{3 P_{0}}{2}\right] \\
& \vee\left[\frac{\lambda_{M}\left(\boldsymbol{M}_{1 H}\right)}{2}\right] \vee\left[\frac{2}{3}\left(E I_{1} \vee E I_{2} \wedge G I_{3}\right)+\frac{\gamma \Gamma \lambda_{M}(\boldsymbol{J})}{4 \varrho_{02}}\right. \\
& \left.+\frac{96 \gamma \Gamma^{3} \lambda_{M}(\boldsymbol{M})}{\varrho_{01}}+384 P_{0} \Gamma^{2}\right] \vee\left[\frac{\lambda_{M}\left(\boldsymbol{M}_{2 H}\right)}{2}\right] \\
& \vee \frac{1}{6}\left(E A \vee G \bar{A}_{1} \vee G \bar{A}_{2}\right) \vee \frac{1}{6}\left(E I_{1} \vee E I_{2} \vee G I_{3}\right),
\end{aligned}
$$

where $\lambda_{m}(\bullet)$ denotes the minimum eigenvalue of $\bullet$. The constants $\gamma, \varrho_{01}$, and $\varrho_{02}$ are chosen such that

$$
c_{1} \geq c_{1}^{\diamond}
$$

where $c_{1}^{\diamond}$ is a positive constant.

Procedure 5.1 To ensure that (49) holds, the constant $\gamma$ is chosen for any given positive constants $\varrho_{01}$ and $\varrho_{02}$ as follows:

$$
\begin{aligned}
\gamma & <\left[\frac{\lambda_{m}(\boldsymbol{M})}{2 \Gamma \varrho_{01} \lambda_{M}(\boldsymbol{M})}\right] \wedge\left[\frac{\lambda_{m}(\boldsymbol{J})}{2 \Gamma \varrho_{02} \lambda_{M}(\boldsymbol{J})}\right] \\
& \wedge\left[\frac{1}{3}\left(E A \wedge G \bar{A}_{1} \wedge G \bar{A}_{2}\right) \frac{2 \varrho_{01}}{\Gamma \lambda_{M}(\boldsymbol{M})}\right] \\
& \wedge\left[\frac{1}{3}\left(E I_{1} \wedge E I_{2} \wedge G I_{3}\right)\left(\frac{\Gamma \lambda_{M}(\boldsymbol{J})}{4 \varrho_{02}}+\frac{96 \Gamma^{3} \lambda_{M}(\boldsymbol{M})}{\varrho_{01}}\right)^{-1}\right]:=\gamma_{0}^{\diamond}
\end{aligned}
$$

Although the positive constants $\varrho_{01}$ and $\varrho_{02}$ can be arbitrary, they should be chosen such that $\gamma_{0}^{\diamond}$ defined in (50) as large as possible to increase the control gains for given $M, \boldsymbol{J}, E A, G \bar{A}_{1}, G \bar{A}_{2}, E I_{1}, E I_{2}$, and $G I_{3}$, see Procedure 5.2.

Thus, $U$ is a proper (positive definite and radially unbounded) functional of $\mathcal{E}$. We now calculate the infinitesimal generator $\mathcal{L} U$. It is obvious from (40) that

$$
\mathcal{L} U=\mathcal{L} U_{0}+\mathcal{L} U_{1}+\mathcal{L} U_{2},
$$

where $\mathcal{L} U_{0}, \mathcal{L} U_{1}$, and $\mathcal{L} U_{2}$ are detailed in the sequel.

Calculation of $\mathcal{L} U_{0}$ : Applying the Itô formula to $U_{0}$ using (42) along the solutions of (37) and (39), and using integration by parts results in (see Appendix B.1):

$$
\begin{aligned}
& \mathcal{L} U_{0}=\left(\boldsymbol{n}^{B \Gamma}-P_{0} \boldsymbol{e}_{3}\right)^{T} \tilde{\boldsymbol{r}}_{t}^{B \Gamma}+\left(\boldsymbol{m}^{B \Gamma}\right)^{T} \boldsymbol{\omega}^{B \Gamma} \\
& \quad-k_{10}^{*}\|\boldsymbol{v}\|_{L^{2}}^{2}-k_{10}^{N *}\left[\left\|v_{1}^{2}\right\|_{L^{2}}^{2}+\left\|v_{2}^{2}\right\|_{L^{2}}^{2}+\left\|v_{3}^{2}\right\|_{L^{2}}^{2}\right] \\
& \quad-k_{20}^{*}\|\boldsymbol{\omega}\|_{L^{2}}^{2}-k_{20}^{N *}\left[\left\|\omega_{1}^{2}\right\|_{L^{2}}^{2}+\left\|\omega_{2}^{2}\right\|_{L^{2}}^{2}+\left\|\omega_{3}^{2}\right\|_{L^{2}}^{2}\right] \\
& \quad+\frac{1}{4 \epsilon_{02}}\left\|\boldsymbol{f}_{10}\right\|_{L^{2}}^{2}+\frac{1}{4 \epsilon_{05}}\left\|\boldsymbol{f}_{20}\right\|_{L^{2}}^{2}+3\left(\delta_{16}^{M}\right)^{2}+4\left(\delta_{28}^{M}\right)^{2},
\end{aligned}
$$

where

$$
\begin{aligned}
k_{10}^{*} & =d_{A 11}^{m}-\lambda_{M}\left(\left|\boldsymbol{M}_{A}\right|\right) \bar{\omega}_{f}^{M}-3 d_{A 12}^{M} \bar{v}_{f}^{M}\left(\epsilon_{01}+\bar{v}_{f}^{M} \epsilon_{02}\right) \\
& -\lambda_{M}\left(\left|\boldsymbol{M}_{A}\right|\right) \bar{v}_{f}^{M} \epsilon_{03}-12 \lambda_{M}^{2}\left(\left|\boldsymbol{M}_{A}\right|\right)\left(\delta_{23}^{M}\right)^{2} \\
& -12 \lambda_{M}^{2}\left(\left|\boldsymbol{M}_{A}\right|\right)\left(\delta_{24}^{M}\right)^{2}-9 \lambda_{M}^{2}\left(\left|\boldsymbol{M}_{A}\right|\right)\left(\delta_{12}^{M}\right)^{2} \\
& -27 d_{A 12}\left(d_{A 12}^{M}\right)^{2}\left(\delta_{13}^{M}\right)^{2}-108 d_{A 12}\left(d_{A 12}^{M}\right)^{2}\left(\delta_{14}^{M}\right)^{2},
\end{aligned}
$$




$$
\begin{aligned}
& k_{10}^{N *}=d_{A 12}^{m}-\frac{9 d_{A 12}^{M} \bar{v}_{f}^{M}}{4 \epsilon_{01}}-81\left(d_{A 12}^{M}\right)^{2}\left(\delta_{15}^{M}\right)^{2}, \\
& k_{20}^{*}=d_{A 21}^{m}-\lambda_{M}\left(\left|\boldsymbol{J}_{A}\right|\right) \bar{\omega}_{f}^{M}-\frac{\lambda_{M}\left(\left|\boldsymbol{M}_{A}\right|\right) \bar{v}_{f}^{M}}{4 \epsilon_{03}} \\
& \quad-3 d_{A 21}^{M} \bar{\omega}_{f}^{M} \epsilon_{04}-\epsilon_{05}-3 d_{A 22}^{M}\left(\bar{\omega}_{f}^{M}\right)^{2}-9 \lambda_{M}^{2}\left(\left|\boldsymbol{M}_{A}\right|\right) \delta_{11}^{M} \\
& \quad-12 \lambda_{M}^{2}\left(\left|\boldsymbol{J}_{A}\right|\right)\left(\left(\delta_{21}^{M}\right)^{2}+\left(\delta_{22}^{M}\right)^{2}\right) \\
& \quad-12\left(d_{A 22}^{M}\right)^{2}\left(\left(\delta_{25}^{M}\right)^{2}+2\left(\delta_{26}^{M}\right)^{2}\right), \\
& k_{20}^{N *}=d_{A 22}^{m}-\frac{9 d_{A 22}^{M} \bar{\omega}_{f}^{M}}{4 \epsilon_{04}}-36\left(d_{A 22}^{M}\right)^{2}\left(\delta_{27}^{M}\right)^{2},
\end{aligned}
$$

with $\epsilon_{0 i}, i=1,3,4$ as in (19) and $\epsilon_{0 i}, i=2,5$ positive constants to be chosen.

Calculation of $\mathcal{L} U_{1}$ : Applying the Itô formula to $U_{1}$ defined in (43) results in

$$
\mathcal{L} U_{1}=\mathcal{L} U_{11}+\mathcal{L} U_{12} .
$$

With $U_{11}$ in (44), we have, see Appendix B.2.1:

$$
\begin{aligned}
\mathcal{L} & U_{11} \leq \gamma \Gamma\left(\boldsymbol{n}^{B \Gamma}-P_{0} \boldsymbol{e}_{3}\right) \mathbb{D} \tilde{\boldsymbol{r}}^{B \Gamma}-\frac{5 \gamma \Gamma}{12}\left[G \bar{A}_{1} \wedge G \bar{A}_{2} \wedge E A\right] \\
& \times\left\|\boldsymbol{\vartheta}^{B \Gamma}\right\|^{2}-\gamma \Gamma P_{0}\left(\varepsilon^{B \Gamma}-\mathbb{D} \tilde{z}^{B \Gamma}\right)-k_{11}^{*}\|\boldsymbol{v}\|_{L^{2}}^{2} \\
& -k_{11}^{N *}\left(\left\|v_{1}^{2}\right\|_{L^{2}}^{2}+\left\|v_{2}^{2}\right\|_{L^{2}}^{2}+\left\|v_{3}^{2}\right\|_{L^{2}}^{2}\right)+k_{21}^{*}\|\boldsymbol{\omega}\|_{L^{2}}^{2} \\
& +k_{21}^{N *}\left(\left\|\omega_{1}^{2}\right\|_{L^{2}}^{2}+\left\|\omega_{2}^{2}\right\|_{L^{2}}^{2}+\left\|\omega_{3}\right\|_{L^{2}}^{2}\right)-k_{31}^{*}\|\boldsymbol{\vartheta}\|_{L^{2}}^{2} \\
& -k_{31}^{N *}\left(\left\|\eta_{1}^{2}\right\|_{L^{2}}^{2}+\left\|\eta_{2}^{2}\right\|_{L^{2}}^{2}+\|\varepsilon\|_{L^{2}}^{2}+k_{41}^{*}\|\boldsymbol{\mu}\|_{L^{2}}^{2}-\left[P_{0}\right.\right. \\
& \left.-\frac{\gamma E A}{2 \epsilon_{13}}-\frac{\gamma E A}{4 \epsilon_{14}}-\frac{2 \gamma E A}{\epsilon_{14}^{4}}\right]\left\langle 1,1-\cos \left(\theta_{1}\right) \cos \left(\theta_{2}\right)\right\rangle_{L^{2}} \\
& -\gamma\langle\boldsymbol{n},(\boldsymbol{\mu} \times \mathbb{D} \boldsymbol{r}) s\rangle_{L^{2}}+\frac{\gamma \Gamma}{4 \epsilon_{112}}\left\|\boldsymbol{f}_{10}\right\|_{L^{2}}^{2},
\end{aligned}
$$

where

$$
\begin{aligned}
k_{11}^{*} & =\gamma\left\{-\frac{\lambda_{m}(\boldsymbol{M})}{2}+\frac{\Gamma \lambda_{M}\left(\left|\boldsymbol{D}_{A 11}\right|\right)}{4 \epsilon_{16}}+\frac{\Gamma \lambda_{M}\left(\left|\boldsymbol{M}_{A}\right|\right) \bar{\omega}_{f}^{M}}{4 \epsilon_{19}}\right. \\
& \left.+\frac{3 \Gamma \lambda_{M}\left(\left|\boldsymbol{D}_{A 12}\right|\left(\bar{v}_{f}^{M}\right)^{2}\right)}{4 \epsilon_{11}}\right\}, \\
k_{11}^{N *} & =\gamma\left\{3 \frac{\Gamma \lambda_{M}(\boldsymbol{M})}{4 \epsilon_{11}}+\frac{48 \Gamma \lambda_{M}\left(\left|\boldsymbol{M}_{A}\right|\right)}{\epsilon_{12}}+3 \Gamma\left[\frac{\lambda_{M}\left(\left|\boldsymbol{M}_{A}\right|\right)}{8 \epsilon_{15}}\right.\right. \\
& \left.\left.+\frac{\lambda_{M}\left(\left|\boldsymbol{D}_{A 12}\right|\right)}{4 \epsilon_{17}^{4}}\right]+\frac{9 \Gamma \lambda_{M}\left(\left|\boldsymbol{D}_{A 12}\right|\right) \bar{v}_{f}^{M}}{4 \epsilon_{110}}\right\}, \\
k_{21}^{*} & =\frac{\gamma \Gamma \lambda_{M}\left(\left|\boldsymbol{M}_{A}\right| \bar{v}_{f}^{M}\right)}{4 \epsilon_{18}}, k_{21}^{N *}=\frac{3 \gamma \Gamma \lambda_{M}\left(\left|\boldsymbol{M}_{A}\right|\right)}{8 \epsilon_{15}}, \\
k_{31}^{*} & =\gamma\left\{\frac{5 G \bar{A}_{1}}{12} \wedge \frac{5 G \bar{A}_{2}}{12} \wedge \frac{5 E A}{12}-2 \Gamma \lambda_{M}\left(\left|\boldsymbol{M}_{A}\right|\right) \epsilon_{12}\right. \\
& -E A \epsilon_{13}-\Gamma\left[\lambda_{M}\left(\left|\boldsymbol{M}_{A}\right|\right) \epsilon_{15}+\lambda_{M}\left(\left|\boldsymbol{D}_{A 11}\right|\right) \epsilon_{16}\right] \\
& -\Gamma\left[\lambda_{M}\left(\left|\boldsymbol{M}_{A}\right|\right)\left(\epsilon_{18} \bar{v}_{f}^{M}+\epsilon_{19} \bar{\omega}_{f}^{M}\right)+3 \lambda_{M}\left(\left|\boldsymbol{D}_{A 12}\right|\right)\right. \\
& \left.\left.\times \bar{v}_{f}^{M}\left(\epsilon_{110}+3 \epsilon_{111} \bar{v}_{f}^{M}\right)+\epsilon_{112}\right]\right\}, \\
k_{31}^{N *} & =\gamma\left\{\frac{G \bar{A}_{1}}{6} \wedge \frac{G \bar{A}_{2}}{6} \wedge \frac{E A}{6}-E A \frac{2 \epsilon_{14 t} \epsilon_{14}^{3}}{4}-\frac{9 \Gamma \lambda_{M}\left(\left|\boldsymbol{D}_{A 12}\right|\right) \epsilon_{17}^{\frac{4}{3}}}{4}\right\}, \\
k_{41}^{*} & =\gamma\left\{16 \Gamma \lambda_{M}(\boldsymbol{M}) \epsilon_{11}+384 \Gamma^{3} \lambda_{M}\left(\left|\boldsymbol{M}_{A}\right| \epsilon_{12}\right)\right\},
\end{aligned}
$$

with $\epsilon_{1 i}, i=1, \ldots, 12$ being positive constants to be chosen. Applying the Itô formula to $U_{12}$ defined in (44) yields, see Appendix B.2.2:

$\mathcal{L} U_{12} \leq \gamma \Gamma\left(\boldsymbol{m}^{B \Gamma}\right)^{T} \boldsymbol{\mu}^{B \Gamma}-\frac{5 \gamma}{12}\left[\Gamma E I_{1} \wedge \Gamma E I_{2} \wedge \Gamma G I_{3}\right]\left\|\boldsymbol{\mu}^{B \Gamma}\right\|^{2}$ $+k_{12}^{*}\|\boldsymbol{v}\|_{L^{2}}^{2}+k_{12}^{N *}\left(\left\|v_{1}^{2}\right\|_{L^{2}}^{2}+\left\|v_{2}^{2}\right\|_{L^{2}}^{2}+\left\|v_{3}^{2}\right\|_{L^{2}}^{2}\right)+k_{22}^{*}\|\boldsymbol{\omega}\|_{L^{2}}^{2}$ $+k_{22}^{N *}\left[\left\|\omega_{1}^{2}\right\|_{L^{2}}^{2}+\left\|\omega_{2}^{2}\right\|_{L^{2}}^{2}+\left\|\omega_{3}^{2}\right\|_{L^{2}}^{2}\right]-k_{42}^{*}\|\boldsymbol{\mu}\|_{L^{2}}^{2}-k_{42}^{N *}\left[\left\|\mu_{1}^{2}\right\|_{L^{2}}^{2}\right.$ $\left.+\left\|\mu_{2}^{2}\right\|_{L^{2}}^{2}+\left\|\mu_{3}^{2}\right\|_{L^{2}}^{2}\right]+\frac{\gamma \Gamma}{4 \epsilon_{210}}\left\|\boldsymbol{f}_{20}\right\|_{L^{2}}^{2}+\gamma\langle\boldsymbol{\mu}, \mathbb{D} \boldsymbol{r} \times \boldsymbol{n}\rangle_{L^{2}}$, where

$$
\begin{aligned}
k_{12}^{*} & =\frac{\gamma \Gamma \lambda_{M}\left(\left|\boldsymbol{M}_{A}\right|\right) \bar{v}_{f}^{M}}{2 \epsilon_{27}}, k_{12}^{N *}=\frac{3 \gamma \Gamma \lambda_{M}\left(\left|\boldsymbol{M}_{A}\right|\right)}{4 \epsilon_{23}}, \\
k_{22}^{*} & =\gamma\left\{-\frac{\lambda_{m}(\boldsymbol{J})}{2}+\frac{\Gamma \lambda_{M}\left(\left|\boldsymbol{D}_{A 21}\right|\right)}{4 \epsilon_{24}}\right. \\
& \left.+\Gamma\left[\frac{\lambda_{M}\left(\left|\boldsymbol{J}_{A}\right|\right) \bar{\omega}_{f}^{M}}{2 \epsilon_{22}}+\frac{3 \lambda_{M}\left(\left|\boldsymbol{D}_{A 22}\right|\right)\left(\bar{\omega}_{f}^{M}\right)^{2}}{4 \epsilon_{2}}\right]\right\}, \\
k_{22}^{N *} & =\gamma\left\{\frac{3 \Gamma \lambda_{M}(\boldsymbol{J})}{2 \epsilon_{21}}+\frac{3 \Gamma \lambda_{M}(\boldsymbol{J})}{4 \epsilon_{22}}+\frac{3 \Gamma \lambda_{M}\left(\left|\boldsymbol{D}_{A 22}\right|\right)}{4 \epsilon_{25}^{4}}\right. \\
& \left.+\frac{9 \Gamma \lambda_{M}\left(\left|\boldsymbol{D}_{A 22}\right|\right) \bar{\omega}_{f}^{M}}{4 \epsilon_{22}}\right\}, \\
k_{42}^{*} & =\gamma\left\{\frac{5 E I_{1}}{12} \wedge \frac{5 I_{2}}{12} \wedge \frac{5 G I_{3}}{12}-2 \Gamma \lambda_{M}(\boldsymbol{J}) \epsilon_{21}-\Gamma \lambda_{M}(\boldsymbol{J}) \epsilon_{22}\right. \\
& -\Gamma\left(\lambda_{M}\left(\left|\boldsymbol{M}_{A}\right|\right) \epsilon_{23}+\lambda_{M}\left(\left|\boldsymbol{D} \boldsymbol{D}_{A 21}\right|\right) \epsilon_{24}\right)-\Gamma\left(2 \lambda_{M}\left(\left|\boldsymbol{J}_{A}\right|\right)\right. \\
& \times \bar{\omega}_{f}^{M} \epsilon_{26}+2 \lambda_{M}\left(\left|\boldsymbol{M}_{A}\right|\right) \bar{v}_{f}^{M} \epsilon_{27}+3 \lambda_{M}\left(\left|\boldsymbol{D}_{A 22}\right|\right) \bar{\omega}_{f}^{M} \epsilon_{28} \\
& \left.\left.+3 \lambda_{M}\left(\left|\boldsymbol{D}_{A 22}\right|\right)\left(\bar{\omega}_{f}^{M}\right)^{2} \epsilon_{29}+\epsilon_{210}\right)\right\}, \\
k_{42}^{N *} & =\gamma\left\{\frac{E I_{1}}{6} \wedge \frac{E I_{2}}{6} \wedge \frac{G I_{3}}{6}-\frac{9 \Gamma \lambda_{M}\left(\left|\boldsymbol{D}_{A 22}\right|\right) \epsilon_{25}^{\frac{4}{3}}}{4}\right\} .
\end{aligned}
$$

with $\epsilon_{2 i}, i=1, \ldots, 10$ positive constants to be chosen.

Calculation of $\mathcal{L} U_{2}$ : Applying the Itô formula to $U_{2}$ defined in (45) along the solutions of (39) with the use of (18) and using Young's inequality result in

$$
\begin{aligned}
& \mathcal{L} U_{2} \leq\left(\gamma \Gamma \mathbb{D} \tilde{\boldsymbol{r}}^{B \Gamma}+\tilde{\boldsymbol{r}}_{t}^{B \Gamma}\right)^{T}\left(\gamma \Gamma \boldsymbol{M}_{1 H} \mathbb{D} \tilde{\boldsymbol{r}}_{t}^{B \Gamma}-\left(\boldsymbol{n}^{B \Gamma}-P_{0} \boldsymbol{e}_{3}\right)\right. \\
& -\boldsymbol{D}_{11 B} \tilde{\boldsymbol{r}}_{t}^{B \Gamma}-\boldsymbol{D}_{12 B}\left(\tilde{\boldsymbol{r}}_{t}^{B \Gamma} \otimes \tilde{\boldsymbol{r}}_{t}^{B \Gamma}\right) \tilde{\boldsymbol{r}}_{t}^{B \Gamma}+\epsilon_{1 B}\left(\gamma \Gamma \mathbb{D} \tilde{\boldsymbol{r}}^{B \Gamma}\right. \\
& \left.\left.+\tilde{\boldsymbol{r}}_{t}^{B \Gamma}\right)+\boldsymbol{\phi}_{1 B}\right)+\left(\gamma \Gamma \boldsymbol{\mu}^{B \Gamma}+\boldsymbol{\omega}^{B \Gamma}\right)^{T}\left(\gamma \Gamma \boldsymbol{M}_{2 H} \boldsymbol{\mu}_{t}^{B \Gamma}\right. \\
& -\boldsymbol{m}^{B \Gamma}-\boldsymbol{D}_{21 B} \boldsymbol{\omega}^{B \Gamma}-\boldsymbol{D}_{22 B}\left(\boldsymbol{\omega}^{B \Gamma} \otimes \boldsymbol{\omega}^{B \Gamma}\right) \boldsymbol{\omega}^{B \Gamma} \\
& \left.+\epsilon_{2 B}\left(\gamma \Gamma \boldsymbol{\mu}^{B \Gamma}+\boldsymbol{\omega}^{B \Gamma}\right)+\boldsymbol{\phi}_{2 B}\right)+\frac{1}{4 \epsilon_{1 B}}\left\|\boldsymbol{f}_{1 B 0}\right\|^{2} \\
& +\frac{1}{4 \epsilon_{2 B}}\left\|\boldsymbol{f}_{2 B 0}\right\|^{2}+\frac{1}{2}\left(\delta_{1 B}^{M}\right)^{2}+\frac{1}{2}\left(\delta_{2 B}^{M}\right)^{2},
\end{aligned}
$$

where $\epsilon_{1 B}$ and $\epsilon_{2 B}$ are positive constants. Now substituting (55) and (57) into (54), then together with (52) and (59) into (51) results in

$$
\begin{aligned}
& \mathcal{L} U \leq-k_{1}\|\boldsymbol{v}\|_{L^{2}}^{2}-k_{1}^{N}\left(\left\|v_{1}^{2}\right\|_{L^{2}}^{2}+\left\|v_{2}^{2}\right\|_{L^{2}}^{2}+\left\|v_{3}^{2}\right\|_{L^{2}}^{2}\right) \\
& \quad-k_{2}\|\boldsymbol{\omega}\|_{L^{2}}^{2}-k_{2}^{N}\left(\left\|\omega_{1}^{2}\right\|_{L^{2}}^{2}+\left\|\omega_{2}^{2}\right\|_{L^{2}}^{2}+\left\|\omega_{3}^{2}\right\|_{L^{2}}^{2}\right) \\
& \quad-k_{3}\|\boldsymbol{v}\|_{L^{2}}^{2}-k_{3}^{N}\left(\left\|\eta_{1}^{2}\right\|_{L^{2}}^{2}+\left\|\eta_{2}^{2}\right\|_{L^{2}}^{2}+\left\|\eta_{3}^{2}\right\|_{L^{2}}^{2}\right) \\
& \quad-k_{4}\|\boldsymbol{\mu}\|_{L^{2}}^{2}-k_{4}^{N}\left(\left\|\mu_{1}^{2}\right\|_{L^{2}}^{2}+\left\|\mu_{2}^{2}\right\|_{L^{2}}^{2}+\left\|\mu_{3}^{2}\right\|_{L^{2}}^{2}\right) \\
& \quad-k_{5}\left\langle 1-\cos \left(\theta_{1}\right) \cos \left(\theta_{2}\right)\right\rangle_{L^{2}}+\Omega_{B}+c_{0}
\end{aligned}
$$

where

$$
\begin{aligned}
k_{1}= & k_{10}^{*}-k_{11}^{*}-k_{12}^{*}, k_{1}^{N}=k_{10}^{N *}-k_{11}^{N *}-k_{12}^{N *}, \\
k_{2}= & k_{20}^{*}-k_{21}^{*}-k_{22}^{*}, k_{2}^{N}=k_{20}^{N *}-k_{21}^{N *}-k_{22}^{N *}, \\
k_{3}= & k_{31}^{*}, k_{3}^{N}=k_{31}^{N *}, k_{4}=k_{42}^{*}-k_{41}^{*}, k_{4}^{N}=k_{42}^{N *}, \\
k_{5}= & \gamma\left[P_{0}-\frac{\gamma E A}{2 \epsilon_{13}}-\frac{\gamma E A}{4 \epsilon_{14}}-\frac{2 \gamma E A}{\epsilon_{14}^{4}}\right], \\
c_{0}= & \frac{1}{4 \epsilon_{00}}\left\|\boldsymbol{f}_{10}\right\|_{L^{2}}^{2}+\frac{1}{4 \epsilon_{0}}\left\|\boldsymbol{f}_{20}\right\|_{L^{2}}^{2}+3\left(\delta_{16}^{M}\right)^{2}+4\left(\delta_{28}^{M}\right)^{2} \\
& +\frac{\gamma \Gamma}{4 \epsilon_{112}}\left\|\boldsymbol{f}_{10}\right\|_{L^{2}}^{2}+\frac{\gamma \Gamma}{4 \epsilon_{210}}\left\|\boldsymbol{f}_{20}\right\|_{L^{2}}^{2}+\frac{1}{4 \epsilon_{1 B}}\left\|\boldsymbol{f}_{1 B 0}\right\|^{2} \\
& +\frac{1}{4 \epsilon_{2 B}}\left\|\boldsymbol{f}_{2 B 0}\right\|^{2}+\frac{1}{2}\left(\delta_{1 B}^{M}\right)^{2}+\frac{1}{2}\left(\delta_{2 B}^{M}\right)^{2},
\end{aligned}
$$

and

$$
\begin{aligned}
& \Omega_{B}=-\frac{5 \gamma \Gamma}{12}\left[G \bar{A}_{1} \wedge G \bar{A}_{2} \wedge E A\right]\left\|\boldsymbol{\vartheta}^{B \Gamma}\right\|^{2} \\
& -\gamma \Gamma P_{0}\left(\varepsilon^{B \Gamma}-\mathbb{D} \tilde{z}^{B \Gamma}\right)-\frac{5 \gamma \Gamma}{12}\left[E I_{1} \wedge E I_{2} \wedge G I_{3}\right]\left\|\boldsymbol{\mu}^{B \Gamma}\right\|^{2} \\
& +\left(\gamma \Gamma \mathbb{D} \tilde{\boldsymbol{r}}^{B \Gamma}+\tilde{\boldsymbol{r}}_{t}^{B \Gamma}\right)^{T}\left(\gamma \Gamma \boldsymbol{M}_{1 H} \mathbb{D} \tilde{\boldsymbol{r}}_{t}^{B \Gamma} \boldsymbol{D}_{11 B} \tilde{\boldsymbol{r}}_{t}^{B \Gamma}-\boldsymbol{D}_{12 B}\right. \\
& \left.\times\left(\tilde{\boldsymbol{r}}_{t}^{B \Gamma} \otimes \tilde{\boldsymbol{r}}_{t}^{B \Gamma}\right) \tilde{\boldsymbol{r}}_{t}^{B \Gamma}+\epsilon_{1 B}\left(\gamma \Gamma \mathbb{D} \tilde{\boldsymbol{r}}^{B \Gamma}+\tilde{\boldsymbol{r}}_{t}^{B \Gamma}\right)+\boldsymbol{\phi}_{1 B}\right) \\
& +\left(\gamma \Gamma \boldsymbol{\mu}^{B \Gamma}+\boldsymbol{\omega}^{B \Gamma}\right)^{T}\left(\gamma \Gamma \boldsymbol{M}_{2 H} \boldsymbol{\mu}_{t}^{B \Gamma}-\boldsymbol{D}_{21 B} \boldsymbol{\omega}^{B \Gamma}-\boldsymbol{D}_{22 B}\right. \\
& \left.\times\left(\boldsymbol{\omega}^{B \Gamma} \otimes \boldsymbol{\omega}^{B \Gamma}\right) \boldsymbol{\omega}^{B \Gamma}+\epsilon_{2 B}\left(\gamma \Gamma \boldsymbol{\mu}^{B \Gamma}+\boldsymbol{\omega}^{B \Gamma}\right)+\boldsymbol{\phi}_{2 B}\right) .
\end{aligned}
$$


From (62), we choose $\phi_{1 B}$ and $\phi_{2 B}$ as:

$$
\begin{aligned}
\phi_{1 B} & =-\left(\boldsymbol{C}_{1 B}+\boldsymbol{I}_{3} \epsilon_{1 B}\right)\left(\gamma \Gamma \mathbb{D} \tilde{\boldsymbol{r}}^{B \Gamma}+\tilde{\boldsymbol{r}}_{t}^{B \Gamma}\right)-\gamma \Gamma \boldsymbol{M}_{1 H} \mathbb{D} \tilde{\boldsymbol{r}}_{t}^{B \Gamma} \\
& +\boldsymbol{D}_{11 B} \tilde{\boldsymbol{r}}_{t}^{B \Gamma}+\boldsymbol{D}_{12 B}\left(\tilde{\boldsymbol{r}}_{t}^{B \Gamma} \otimes \tilde{\boldsymbol{r}}_{t}^{B \Gamma}\right) \tilde{\boldsymbol{r}}_{t}^{B \Gamma} \\
\boldsymbol{\phi}_{2 B} & =-\left(\boldsymbol{C}_{2 B}+\boldsymbol{I}_{3} \epsilon_{2 B}\right)\left(\gamma \Gamma \boldsymbol{\mu}^{B \Gamma}+\boldsymbol{\omega}^{B \Gamma}\right)-\gamma \Gamma \boldsymbol{M}_{2 H} \boldsymbol{\mu}_{t}^{B \Gamma} \\
& +\boldsymbol{D}_{21 B} \boldsymbol{\omega}^{B \Gamma}+\boldsymbol{D}_{22 B}\left(\boldsymbol{\omega}^{B \Gamma} \otimes \boldsymbol{\omega}^{B \Gamma}\right) \boldsymbol{\omega}^{B \Gamma},
\end{aligned}
$$

where $\boldsymbol{C}_{1 B}$ and $\boldsymbol{C}_{2 B}$ are positive definite matrices to be chosen, and $\boldsymbol{I}_{3}$ is the $3 \times 3$ identity matrix. Note that $\phi_{1 B}$ and $\phi_{2 B}$ need only information from the top-end. Substituting (63) into (62) yields

$$
\begin{aligned}
& \Omega_{B} \leq-\frac{5 \gamma \Gamma}{12}\left[G \bar{A}_{1} \wedge G \bar{A}_{2} \wedge E A\right]\left\|\boldsymbol{\vartheta}^{B \Gamma}\right\|^{2}-\gamma \Gamma P_{0}\left(\varepsilon^{B \Gamma}\right. \\
& \quad-\tilde{z} \bar{B})+\frac{\gamma \Gamma \lambda_{M}\left(\boldsymbol{J}_{0}\right)}{2}\left\|\boldsymbol{\omega}^{B \Gamma}\right\|^{2}+\frac{\gamma \Gamma m_{0}}{2}\left\|\tilde{\boldsymbol{r}}_{t}^{B \Gamma}\right\|^{2} \\
& \quad-\frac{5 \gamma \Gamma}{12}\left[E I_{1} \wedge E I_{2} \wedge G I_{3}\right]\left\|\boldsymbol{\mu}^{B \Gamma}\right\|^{2}-\lambda_{m}\left(\boldsymbol{C}_{1 B}\right) \\
& \quad \times\left\|\gamma \Gamma \mathbb{D} \tilde{\boldsymbol{r}}^{B \Gamma}+\tilde{\boldsymbol{r}}_{t}^{B \Gamma}\right\|^{2}-\lambda_{m}\left(\boldsymbol{C}_{2 B}\right)\left\|\gamma \Gamma \boldsymbol{\mu}^{B \Gamma}+\boldsymbol{\omega}^{B \Gamma}\right\|^{2}
\end{aligned}
$$

Let us consider the term

$$
\Psi_{B}=-\delta_{1 B}\left\|\gamma \Gamma \mathbb{D} \tilde{\boldsymbol{r}}^{B \Gamma}+\tilde{\boldsymbol{r}}_{t}^{B \Gamma}\right\|^{2}-\delta_{2 B}\left\|\gamma \Gamma \boldsymbol{\mu}^{B \Gamma}+\boldsymbol{\omega}^{B \Gamma}\right\|^{2},
$$

where $\delta_{1 B}$ and $\delta_{2 B}$ are positive constants to be chosen. Expanding the right hand side of (65) and using Young's inequality and the first equation of (24), we have:

$$
\begin{aligned}
& \Psi_{B} \leq-\delta_{1 B} \gamma^{2} \Gamma^{2}\left(1-\frac{1}{\varrho_{1 B}}\right)\left\|\boldsymbol{\vartheta}^{B \Gamma}\right\|^{2}-\delta_{2 B} \gamma^{2} \Gamma^{2}\left(1-\frac{1}{\varrho_{2 B}}\right) \\
& \quad \times\left\|\boldsymbol{\mu}^{B \Gamma}\right\|^{2}-2 \delta_{1 B} \gamma^{2} \Gamma^{2}\left(1-\frac{1}{\varrho_{1 B}}\right)\left(\varepsilon^{B \Gamma}-\mathbb{D} \tilde{z}^{B \Gamma}\right) \\
& \quad-\delta_{1 B}\left(1-\varrho_{1 B}\right)\left\|\tilde{\boldsymbol{r}}_{t}^{B \Gamma}\right\|^{2}-\delta_{2 B}\left(1-\varrho_{2 B}\right)\left\|\boldsymbol{\omega}^{B \Gamma}\right\|^{2},
\end{aligned}
$$

where $\varrho_{1 B}$ and $\varrho_{2 B}$ are positive constants to be chosen. With the use of (66), we can write (64) as follows:

$$
\begin{aligned}
& \Omega_{B} \leq-k_{11 B}\left\|\tilde{\boldsymbol{r}}_{t}^{B \Gamma}\right\|^{2}-k_{12 B}\left\|\boldsymbol{\vartheta}^{B \Gamma}\right\|^{2}-k_{13 B}\left(\varepsilon^{B \Gamma}-\tilde{z}^{B \Gamma}\right) \\
& \quad k_{21 B}\left\|\boldsymbol{\omega}^{B \Gamma}\right\|^{2}-k_{22 B}\left\|\boldsymbol{\mu}^{B \Gamma}\right\|^{2}-k_{31 B}\left\|\gamma \Gamma \mathbb{D} \tilde{\boldsymbol{r}}^{B \Gamma}+\tilde{\boldsymbol{r}}_{t}^{B \Gamma}\right\|^{2} \\
& \quad-k_{32 B}\left\|\gamma \Gamma \boldsymbol{\mu}^{B \Gamma}+\boldsymbol{\omega}^{B \Gamma}\right\|^{2}
\end{aligned}
$$

where

$$
\begin{aligned}
& k_{11 B}=\delta_{1 B}\left(1-\varrho_{1 B}\right)-\frac{\gamma \Gamma m_{0}}{2} \\
& k_{12 B}=\gamma \Gamma\left\{\frac{5}{12}\left[G \bar{A}_{1} \wedge G \bar{A}_{2} \wedge E A\right]+\delta_{1 B} \gamma \Gamma\left(1-\frac{1}{\varrho_{1 B}}\right)\right\} \\
& k_{13 B}=\gamma \Gamma\left[P_{0}+2 \delta_{1 B} \gamma \Gamma\left(1-\frac{1}{\varrho_{1 B}}\right)\right] \\
& k_{21 B}=\delta_{2 B}\left(1-\varrho_{2 B}\right)-\frac{\gamma \Gamma \lambda_{M}\left(\overline{\boldsymbol{J}}_{0}\right)}{2} \\
& k_{22 B}=\gamma \Gamma\left\{\frac{5}{12}\left[E I_{1} \wedge E I_{2} \wedge G I_{3}\right]+\delta_{2 B} \gamma \Gamma\left(1-\frac{1}{\varrho_{2 B}}\right)\right\} \\
& k_{31 B}=\lambda_{m}\left(\boldsymbol{C}_{1 B}\right)-\delta_{1 B}, \quad k_{32 B}=\lambda_{m}\left(\boldsymbol{C}_{2 B}\right)-\delta_{2 B}
\end{aligned}
$$

Substituting (67) into (60) yields

$$
\begin{aligned}
& \mathcal{L} U \leq-k_{1}\|\boldsymbol{v}\|_{L^{2}}^{2}-k_{1}^{N}\left(\left\|v_{1}^{2}\right\|_{L^{2}}^{2}+\left\|v_{2}^{2}\right\|_{L^{2}}^{2}+\left\|v_{3}^{2}\right\|_{L^{2}}^{2}\right) \\
& -k_{2}\|\boldsymbol{\omega}\|_{L^{2}}^{2}-k_{2}^{N}\left(\left\|\omega_{1}^{2}\right\|_{L^{2}}^{2}+\left\|\omega_{2}^{2}\right\|_{L^{2}}^{2}+\left\|\omega_{3}^{2}\right\|_{L^{2}}^{2}\right) \\
& -k_{3}\|\boldsymbol{\vartheta}\|_{L^{2}}^{2}-k_{3}^{N}\left(\left\|\eta_{1}^{2}\right\|_{L^{2}}^{2}+\left\|\eta_{2}^{2}\right\|_{L^{2}}^{2}+\left\|\varepsilon^{2}\right\|_{L^{2}}^{2}\right) \\
& -k_{4}\|\boldsymbol{\mu}\|_{L^{2}}^{2}-k_{4}^{N}\left(\left\|\mu_{1}^{2}\right\|_{L^{2}}^{2}+\left\|\mu_{2}^{2}\right\|_{L^{2}}^{2}+\left\|\mu_{3}^{2}\right\|_{L^{2}}^{2}\right) \\
& -k_{5}\left\langle 1-\cos \left(\theta_{1}\right) \cos \left(\theta_{2}\right)\right\rangle_{L^{2}}-k_{11 B}\left\|\tilde{\boldsymbol{r}}_{t}^{B \Gamma}\right\|^{2} \\
& -k_{12 B}\left\|\boldsymbol{\vartheta}^{B \Gamma}\right\|^{2}-k_{13 B}\left(\varepsilon^{B \Gamma}-\tilde{z}^{B \Gamma}\right)-k_{21 B}\left\|\boldsymbol{\omega}^{B \Gamma}\right\|^{2} \\
& -k_{22 B}\left\|\boldsymbol{\mu}^{B \Gamma}\right\|^{2}-k_{31 B}\left\|\gamma \Gamma \mathbb{D} \tilde{\boldsymbol{r}}^{B \Gamma}+\tilde{\boldsymbol{r}}_{t}^{B \Gamma}\right\|^{2} \\
& -k_{32 B}\left\|\gamma \Gamma \boldsymbol{\mu}^{B \Gamma}+\boldsymbol{\omega}^{B \Gamma}\right\|^{2}+c_{0} .
\end{aligned}
$$

We choose $P_{0} ; \gamma ; \epsilon_{0 i}, i=2,5 ; \epsilon_{1 i}, i=1, \ldots, 12 ; \epsilon_{2 i}, i=$ $1, \ldots, 10 ; \delta_{1 B}, \delta_{2 B} ; \varrho_{1 B}, \varrho_{2 B} ; \epsilon_{1 B}, \epsilon_{2 B} ;$ and $\boldsymbol{C}_{i B}, \boldsymbol{C}_{2 B}$ such that

$$
\begin{aligned}
& k_{i} \geq k_{i}^{\diamond}, k_{i}^{N} \geq k_{i}^{N \diamond}, i=1, \ldots, 4, \quad k_{5} \geq 0, \\
& k_{3 i B} \geq k_{3 i B}^{\diamond}, i=1,2, k_{i j B} \geq 0, i=1,2 ; j=1,2,3,
\end{aligned}
$$

where $k_{i}^{\diamond}, k_{i}^{N \diamond}$, and $k_{3 i B}^{\diamond}$ are positive constants.

Procedure 5.2 To ensure that (70) holds, the choice of $P_{0} ; \gamma ; \epsilon_{0 i}, i=2,5 ; \epsilon_{1 i}, i=1, \ldots, 12 ; \epsilon_{2 i}, i=1, \ldots, 9$; $\delta_{1 B}, \delta_{2 B} ; \varrho_{1 B}, \varrho_{2 B} ; \epsilon_{1 B}, \epsilon_{2 B} ;$ and $\boldsymbol{C}_{i B}, \boldsymbol{C}_{2 B}$ is as follows:

Step 1. Choose small $\left(\epsilon_{12}, \epsilon_{13}, \epsilon_{15}, \epsilon_{16}, \epsilon_{18}, \ldots, \epsilon_{112}\right)$ and $\left(\epsilon_{14}, \epsilon_{17}\right)$ so that $k_{31}^{*}$ and $k_{31}^{N *}$ defined in (56) are strictly positive. This makes $k_{31}$ and $k_{31}^{N}$ strictly positive because $k_{3}=k_{31}^{*}$ and $k_{3}^{N}=k_{31}^{N *}$, see (61).

Step 2. Choose small $\left(\epsilon_{11}, \epsilon_{12}, \epsilon_{2 i}\right), i=1, . .4,6, \ldots, 10$ to make $k_{42}^{*}-k_{41}^{*}$ strictly positive, where $k_{41}^{*}$ and $k_{42}^{*}$ are defined in (56) and (58), respectively. This makes $k_{4}$ strictly positive since $k_{4}=k_{42}^{*}-k_{41}^{*}$, see (61). Next, choose small $\epsilon_{25}$ such that $k_{42}^{N *}$ is strictly positive. This makes $k_{4}^{N}$ strictly positive because $k_{4}^{N}=k_{42}^{N *}$, see (61). The resulting $\epsilon_{12}$ is the smallest value in Step 1 and this step.

Step 3. Choose small $\left(\epsilon_{02}, \epsilon_{05}\right)$ such that $k_{10}^{*}$ and $k_{20}^{*}$ defined in (53) are strictly positive. This is feasible because of the first and third inequalities in (19). Thus, all the constants $\left(k_{10}^{*}, k_{10}^{N *}, k_{20}^{*}, k_{20}^{N *}\right)$ defined in (53) are now strictly positive due to the inequalities in (19).

Step 4. With all $\epsilon_{1 i}, i=1, \ldots, 12, \epsilon_{2 i}, i=1, \ldots, 10$, and $\left(\epsilon_{02}, \epsilon_{05}\right)$ chosen in Steps 1-3, choose $\gamma$ such that such that $\left(k_{1}, k_{1}^{N}, k_{2}, k_{2}^{N}\right)$ defined in (61) are strictly positive. This is feasible due to the expression of $\left(k_{1}, k_{1}^{N}, k_{2}, k_{2}^{N}\right)$ in (61), where $\left(k_{10}^{*}, k_{10}^{N *}, k_{20}^{*}, k_{20}^{N *}\right)$ are strictly positive as chosen in Step 3, and all the $\left(k_{i j}^{*}, k_{i j}^{N *}\right),(i, j)=1,2$ have $\gamma$ as a factor, see (56) and (58). This step results in four values of $\gamma$ denoted by $\gamma_{i}^{\diamond}, i=1, \ldots, 4$.

Step 5. Choose $\left(\varrho_{i B}, \delta_{i B}\right), i=1,2$ to make $\delta_{i B}\left(1-\varrho_{i B}\right)$ strictly positive. Then choose $\gamma$ such that $\left(k_{11 B}, k_{21 B}\right)$ defined in (68) are nonnegative. Next, choose $\gamma$ so that $\left(k_{12 B}, k_{22 B}\right)$ defined in (68) are nonnegative. This step results in four values of $\gamma$ denoted by $\gamma_{i}^{\diamond}, i=5, \ldots, 8$. The desired $\gamma$ is the one that is strictly less than the minimum value of $\gamma_{i}^{\diamond}, i=1, \ldots, 8$ and $\gamma_{0}^{\diamond}$ defined in (50).

Step 6. With $\left(\epsilon_{13}, \epsilon_{14}\right)$ chosen in Step 1 and $\gamma$ chosen in Step 5, choose $P_{0}$ such that $k_{5}$ defined in (61) is strictly positive. Next, with $\left(\delta_{1 B}, \varrho_{1 B}, \gamma\right)$ chosen in Step 5 , choose $P_{0}$ such that $k_{13 B}$ defined in (68) is nonnegative. This step results in two values of $P_{0}$ denoted by $P_{0 i}^{\diamond}, i=1,2$. The desired $P_{0}$ is the maximum value of $P_{0 i}^{\diamond}, i=1,2$.

Step 7. With $\delta_{i B}, i=1,2$ chosen in Step 5, choose $\boldsymbol{C}_{i B}, i=1,2$ such that $k_{3 i B}, i=1,2$ are strictly positive. Note that $\left(\epsilon_{1 B}, \epsilon_{2 B}\right)$ should be large to reduce $c_{0}$, see (61).

With (70), we can further write (69) as follows:

$$
\mathcal{L} U \leq-c_{3} \mathcal{E}+c_{0}
$$

where $c_{0}$ is given in (61) and

$$
c_{3}=k_{1} \wedge k_{2} \wedge k_{3} \wedge k_{3}^{N} \wedge k_{4} \wedge k_{4}^{N} \wedge k_{31 B} \wedge k_{32 B} .
$$


We summarize the main results in the following theorem.

Theorem 5.1 Under Assumption 3.1 (particularly the condition (19)), the boundary control vectors $\phi_{i B}, i=$ 1,2 given in (63) solve Control Objective 3.1 provided that $P_{0} ; \gamma ; \varrho_{0 i}, i=1,2 ; \epsilon_{0 i}, i=2,5 ; \epsilon_{1 i}, i=1, \ldots, 12$; $\epsilon_{2 i}, i=1, \ldots, 10 ; \delta_{i B}, i=1,2 ; \varrho_{i B}, i=1,2 ; \epsilon_{i B}, i=1,2$; and $\boldsymbol{C}_{i B}, i=1,2$ are chosen such that the conditions (49) and (70) hold, see Procedures 5.1 and 5.2. The closedloop system consisting of (37), (39), and (63) is globally well-posed and globally practically exponentially p-stable at the origin. In particular,

$$
\mathbb{E}\{\mathcal{E}(t)\} \leq \frac{c_{2}}{c_{1}} \mathcal{E}\left(t_{0}\right) e^{-c_{3}\left(t-t_{0}\right)}+\frac{c_{0}}{c_{1} c_{3}},
$$

where $c_{0}, c_{1}, c_{2}, c_{3}$ are given in (61), (48), and (72).

Proof. Define $\boldsymbol{X}=\operatorname{col}\left(\tilde{\boldsymbol{r}}, \boldsymbol{q}, \boldsymbol{v}, \boldsymbol{\omega}, \tilde{\boldsymbol{r}}^{B \Gamma}, \boldsymbol{q}^{B \Gamma}, \tilde{\boldsymbol{r}}_{t}^{B \Gamma}, \boldsymbol{\omega}^{B \Gamma}\right)$, $\boldsymbol{F}(\boldsymbol{X}, t)=\operatorname{col}\left(\boldsymbol{R}_{1}(\boldsymbol{q}) \boldsymbol{v}, \boldsymbol{K}(\boldsymbol{q}) \boldsymbol{\omega}, \overline{\boldsymbol{F}}_{1}, \overline{\boldsymbol{F}}_{2}, \tilde{\boldsymbol{r}}_{t}^{B \Gamma}, \boldsymbol{K}\left(\boldsymbol{q}^{B \Gamma}\right) \boldsymbol{\omega}^{B \Gamma}\right.$, $\left.\overline{\boldsymbol{F}}_{1 B}, \overline{\boldsymbol{F}}_{2 B}\right), \boldsymbol{G}(\boldsymbol{X}, t)=\operatorname{col}\left(0,0, \overline{\boldsymbol{G}}_{1}, \overline{\boldsymbol{G}}_{2}, 0,0, \overline{\boldsymbol{G}}_{1 B}, \overline{\boldsymbol{G}}_{2 B}\right)$, where $\overline{\boldsymbol{F}}_{i}, \overline{\boldsymbol{G}}_{i}, \overline{\boldsymbol{F}}_{i B}$, and $\overline{\boldsymbol{G}}_{i B}, i=1,2$ are defined in (37) and (39) with $\phi_{i B}, i=1,2$ being defined in (63). Then, we can write (37) and (39) as (25). We now introduce the functional spaces: $H=\left(W^{2,2}(\mathcal{D})\right)^{6} \times\left(W^{1,2}(\mathcal{D})\right)^{6} \times$ $\mathbb{R}^{12}, V=\left(W_{0}^{2,2}(\mathcal{D})\right)^{6} \times\left(W_{0}^{1,2}(\mathcal{D})\right)^{6} \times \mathbb{R}^{12}, V^{*}=$ $\left(W^{-2,2}(\mathcal{D})\right)^{6} \times\left(W^{-1,2}(\mathcal{D})\right)^{6} \times \mathbb{R}^{12}$, where $\mathcal{D}:=(0, \Gamma)$, $W^{-m, p}(\mathcal{D})$ denotes the dual of $W^{m, p}(\mathcal{D}) ; W_{0}^{2,2}(\mathcal{D})$ and $W_{0}^{1,2}(\mathcal{D})$ denotes $W^{2,2}$ - and $W^{1,2}$-spaces satisfying the first two equations of the boundary conditions (39). Then, we have the embedding $V \subset H \equiv H^{*} \subset V^{*}$. Let $\widehat{\boldsymbol{X}}(t)=\operatorname{col}\left(\widehat{\widetilde{\boldsymbol{r}}}, \widehat{\boldsymbol{q}}, \widehat{\boldsymbol{v}}, \widehat{\boldsymbol{\omega}}, \widehat{\tilde{\boldsymbol{r}}}^{B \Gamma}, \widehat{\boldsymbol{q}}^{B \Gamma}, \widehat{\tilde{\boldsymbol{r}}}_{t}^{B \Gamma}, \widehat{\boldsymbol{\omega}}^{B \Gamma}\right)$. Define

$\langle\boldsymbol{X}, \widehat{\boldsymbol{X}}\rangle_{V}=\frac{1}{2}\langle\boldsymbol{v}, \boldsymbol{M} \widehat{\boldsymbol{v}}\rangle_{L^{2}}+\frac{1}{2}\langle\boldsymbol{\omega}, \boldsymbol{J} \widehat{\boldsymbol{\omega}}\rangle_{L^{2}}+\frac{G \bar{A}_{1}}{2}\left\langle\eta_{1}, \widehat{\eta}_{1}\right\rangle_{L^{2}}$ $+\frac{G \bar{A}_{2}}{2}\left\langle\eta_{2}, \widehat{\eta}_{2}\right\rangle_{L^{2}}+\frac{E A}{2}\langle\varepsilon, \widehat{\varepsilon}\rangle_{L^{2}}+\frac{E I_{1}}{2}\left\langle\mu_{1}, \widehat{\mu}_{1}\right\rangle_{L^{2}}+\frac{E I_{2}}{2}$

$\times\left\langle\mu_{2}, \widehat{\mu}_{2}\right\rangle_{L^{2}}+\frac{G I_{3}}{2}\left\langle\mu_{3}, \widehat{\mu}_{3}\right\rangle_{L^{2}}+\frac{G \bar{A}_{1}}{4}\left\langle\eta_{1}^{2}, \widehat{\eta}_{1}^{2}\right\rangle_{L^{2}}+\frac{G \bar{A}_{2}}{4}$

$\times\left\langle\eta_{2}^{2}, \widehat{\eta}_{2}^{2}\right\rangle_{L^{2}}+\frac{E A}{4}\left\langle\varepsilon^{2}, \widehat{\varepsilon}^{2}\right\rangle_{L^{2}}+\frac{E I_{1}}{4}\left\langle\mu_{1}^{2}, \widehat{\mu}_{1}^{2}\right\rangle_{L^{2}}+\frac{E I_{2}}{4}$

$\times\left\langle\mu_{2}^{2}, \widehat{\mu}_{2}^{2}\right\rangle_{L^{2}}+\frac{G I_{3}}{4}\left\langle\mu_{3}^{2}, \widehat{\mu}_{3}^{2}\right\rangle_{L^{2}}+\frac{P_{0}}{2}\langle 1, \varepsilon-\mathbb{D} \tilde{z}\rangle_{L^{2}}+\frac{P_{0}}{2}$ $\times\langle 1, \widehat{\varepsilon}-\mathbb{D} \widehat{\tilde{z}}\rangle_{L^{2}}+\frac{\gamma}{2}\left\langle\mathbb{D} \tilde{\boldsymbol{r}}, \boldsymbol{R}_{1} \boldsymbol{M} \widehat{\boldsymbol{v}} s\right\rangle_{L^{2}}+\frac{\gamma}{2}\left\langle\mathbb{D} \widehat{\tilde{\boldsymbol{r}}}, \boldsymbol{R}_{1} \boldsymbol{M} \boldsymbol{v} s\right\rangle_{L^{2}}$ $+\frac{\gamma}{2}\left\langle\boldsymbol{\mu}, \mathbb{D}\left(\boldsymbol{J}_{0} \widehat{\boldsymbol{\omega}}\right) s\right\rangle_{L^{2}}+\frac{1}{2}\left(\gamma \Gamma \mathbb{D} \tilde{\boldsymbol{r}}^{B \Gamma_{+}}+\tilde{\boldsymbol{r}}_{t}^{B \Gamma}\right)^{T} \boldsymbol{M}_{1 H}\left(\gamma \Gamma \mathbb{D} \widehat{\widetilde{\boldsymbol{r}}}^{B \Gamma}+\widehat{\tilde{\boldsymbol{r}}}_{t}^{B \Gamma}\right)$ $+\frac{\gamma}{2}\left\langle\widehat{\boldsymbol{\mu}}, \mathbb{D}\left(\boldsymbol{J}_{0} \boldsymbol{\omega}\right) s\right\rangle_{L^{2}}+\frac{1}{2}\left(\gamma \Gamma \boldsymbol{\mu}^{B \Gamma}+\boldsymbol{\omega}^{B \Gamma}\right){ }^{T} \boldsymbol{M}_{2 H}\left(\gamma \Gamma \widehat{\boldsymbol{\mu}}^{B \Gamma}+\widehat{\boldsymbol{\omega}}^{B \Gamma}\right)$,

where $\widehat{\eta}_{i}, i=1,2, \widehat{\mu}_{i}, i=1,2,3$, and $\widehat{\varepsilon}$ are the values of $\eta_{i}, i=1,2, \mu_{i}, i=1,2,3$, and $\varepsilon$ with $\mathbb{D} \tilde{\boldsymbol{r}}$ and $\boldsymbol{q}$ being replaced by $\mathbb{D} \widehat{\tilde{\boldsymbol{r}}}$ and $\widehat{\boldsymbol{q}}$, respectively. The constant $\gamma$ and the constant axial force $P_{0}$ satisfy the conditions specified in Theorem 5.1. Let us denote by $\langle\boldsymbol{X}, \widehat{\boldsymbol{X}}\rangle_{L V}$ linearization of $\langle\boldsymbol{X}, \widehat{\boldsymbol{X}}\rangle_{V}$ at the origin. Then, it can be verified that $\langle\boldsymbol{X}, \widehat{\boldsymbol{X}}\rangle_{L V}$ is a inner product with the norm $\langle\boldsymbol{X}, \boldsymbol{X}\rangle_{L V}=$ $\|\boldsymbol{X}\|_{L V}^{2}$. In fact, there exist strictly positive constants $\bar{c}_{01}$ and $\bar{c}_{02}$ such that $\bar{c}_{01} \mathcal{E}_{L V} \leq\|\boldsymbol{X}\|_{L V}^{2} \leq \bar{c}_{02} \mathcal{E}_{L H}$ locally, where $\mathcal{E}_{L V}$ is the linearization of $\mathcal{E}$, which is defined in (22). Now, to prove Theorem 5.1 we just need to verify all the conditions of Theorem 4.1. The continuity condition in Assumption 4.1 holds due to continuity of $\boldsymbol{F}(\boldsymbol{X}, t)$ and $\boldsymbol{Q}(\boldsymbol{X}, t)$. By using $\langle\boldsymbol{X}-\overline{\boldsymbol{X}}, \boldsymbol{F}(\boldsymbol{X}, t)-\boldsymbol{F}(\overline{\boldsymbol{X}}, t)\rangle_{V, V^{*}}=$ $\langle\boldsymbol{X}-\overline{\boldsymbol{X}}, \boldsymbol{F}(\boldsymbol{X}, t)-\boldsymbol{F}(\overline{\boldsymbol{X}}, t)\rangle_{H}$ with the use of the local inner product in $L V$ defined as above and integration by parts similarly to the calculation of $\mathcal{L} U$ in Section 5.2, it is readily shown that all the local conditions in Assumption 4.1 hold. From (47) and (71) with $\mathcal{E}$ defined in (22), it is clear that the conditions $(31),(32),(34)$, and (35) hold. Thus, proof of Theorem 5.1 is completed.

\section{Simulation results}

This section illustrates the effectiveness of the proposed boundary controller on a slender marine riser with [3]: $\Gamma=500 \mathrm{~m}$; outer diameter $d_{o}=0.61 \mathrm{~m}$; inner diameter $d_{\text {in }}=0.41 \mathrm{~m} ; \varrho=7850 \mathrm{~kg} / \mathrm{m}^{3} ; E=2.04 \times 10^{10} \mathrm{~kg} / \mathrm{m}^{2}$; and $G=8.4 \times 10^{10} \mathrm{~kg} / \mathrm{m}^{2}$. We take $\boldsymbol{M}_{A}=-0.5 \boldsymbol{I}_{3} m_{0}$; $\boldsymbol{J}_{A}=0.5 \boldsymbol{J}_{0} ; \boldsymbol{M}_{1 H}=1000 \boldsymbol{I}_{3} \mathrm{~kg} ; \boldsymbol{M}_{2 H}=500 \boldsymbol{I}_{3} \mathrm{~kg} / \mathrm{m}$; $\boldsymbol{D}_{11 B}=500 \boldsymbol{I}_{3} \mathrm{~kg} / \mathrm{s} ;$ and $\boldsymbol{D}_{21 B}=250 \mathrm{kgm}^{2} / \mathrm{s} ; \boldsymbol{D}_{12 B}$ and $\boldsymbol{D}_{22 B}$ equal to $50 \%$ of $\boldsymbol{D}_{11 B}$ and $\boldsymbol{D}_{21 B}$. For the fluid-loading due to viscous effects that include both boundary-layer skin friction and separation that generates wake drag, the one-degree-of-freedom approach in [42], [49] is extended to riser motion in $3 \mathrm{D}$ space. The fluid motion is decomposed into its orthogonal components perpendicular and a component parallel to the axis of the cylinder. The load due to cross-flow is approximated by bluff-body analysis requiring empirically-based drag coefficients while the load due to the axial-flow is obtained approximately by boundary-layer theory; in both models drag coefficients are chosen for Reynolds numbers typical of the present application. The resulting expressions for $\boldsymbol{D}_{A 11}=-\operatorname{diag}\left(d_{A 11}, d_{A 12}, d_{A 13}\right)$ and $\boldsymbol{D}_{A 21}=-\operatorname{diag}\left(d_{A 21}, d_{A 22}, d_{A 23}\right)$ are as follows:

$$
d_{A k i}=C_{L k i}+C_{D k i} \frac{\varrho_{w} d_{o}}{2} \sqrt{8 / \pi} \sigma_{k i}(s, t),
$$

for $k=1,2$ and $i=1,2,3$, where $\varrho_{w}$ is the water density; $C_{L k i}$ are the linear viscous damping coefficients; $C_{D k i}$ are the skin-friction drag coefficients; $\sigma_{i}(s, t)$ is the root mean square of water particle velocity. The nonlinear damping coefficient matrices $\boldsymbol{D}_{12}$ and $\boldsymbol{D}_{22}$ taken to be $50 \%$ of $\boldsymbol{D}_{11}$ and $\boldsymbol{D}_{21}$, respectively. The coefficients of distributed damping and fluid loads are taken as $C_{L 11}=$ $C_{L 12}=120 \frac{\mathrm{Ns}}{\mathrm{m}}, C_{L 13}=60 \mathrm{Ns}, C_{D 11}=C_{D 12}=1.2$, $C_{D 13}=0.6 ; C_{L 21}=C_{L 22}=90 \mathrm{Ns}, C_{L 23}=60 \mathrm{Nms}$, $C_{D 21}=C_{D 22}=0.8, C_{D 23}=0.4$; and sea-water density is $\varrho_{w}=1025 \mathrm{~kg} / \mathrm{m}^{3}$. Using linear wave theory, the water flow velocities $\boldsymbol{v}_{f}$ and $\boldsymbol{\omega}_{f}$ are defined as $\boldsymbol{v}_{f}=$ $\operatorname{col}\left(\vartheta_{f 1}, \vartheta_{f 2}, \vartheta_{f 3}\right)$ and $\boldsymbol{\omega}_{f}=\operatorname{col}\left(\vartheta_{f 4}, \vartheta_{f 5}, \vartheta_{f 6}\right)$ with [42]:

$$
\vartheta_{f i}(s, t)=\sum_{j=1}^{N_{i}} A_{i j} \omega_{i j} \frac{\cosh \left(k_{i j} s\right)}{\sinh \left(k_{i j} L\right)}\left[\sin \left(\omega_{i j} t\right)+B_{i} \frac{d w_{i}}{d t}\right],
$$

where $w_{i}$ is the standard Wiener process; $B_{i}$ is the coefficient; amplitude $A_{i j}$, wave number $k_{i j}$, frequency $\omega_{i j}$ of the wave $j$ are

$\omega_{i j}=\omega_{i m}+\frac{\omega_{i M}-\omega_{i m}}{N_{i}} j, S_{i j}=\frac{1.25}{4} \frac{\omega_{i o}^{4}}{\omega_{j}^{5}} H_{i}^{2} e^{-1.25\left(\frac{\omega_{i o}}{\omega_{j}}\right)^{4}}$

$A_{i j}=\sqrt{2 S_{i j} \frac{\omega_{i M}-\omega_{i m}}{N_{i}}}, 9.8 k_{i j} \tanh \left(k_{i j} L\right)=\omega_{i j}^{2}$.

In $(77), \omega_{i m}=0.2 \mathrm{rand} / \mathrm{s}, \omega_{i M}=2.5 \mathrm{rand} / \mathrm{s}$; the twoparameter Bretschneider spectrum $S_{i j}$ are used with the significant wave heights $H_{i}$; the modal frequency is $\omega_{i o}=$ 
$\frac{2 \pi}{T_{i}}$ with $T_{1}=T_{2}=7.8, T_{3}=5 ; N_{i}=10$. The significant wave heights $H_{i}=4 \mathrm{~m}$ and the coefficients $B_{i}=0.01$ so that $d_{A k j}^{m}=200 \mathrm{Ns} / \mathrm{m}, d_{A k j}^{M}=280 \mathrm{Ns} / \mathrm{m}, k=1,2, j=$ $1,2,3$, and all the $\delta_{1 i}^{M}, i=1, . .6 \delta_{2 i}^{M}, i=1, . .8$ and are equal to $8 \%$ of $d_{A 11}^{m}$ and $d_{A 21}^{m}$, respectively. We take $\boldsymbol{\Delta}_{i B}=50 \boldsymbol{I}_{3}, i=1,2$. The above choice ensures that the condition (19) holds. We run three cases: 1) Only tension $P_{0}$ is applied, i.e., we set the boundary controls $\phi_{1 B}=$ $\operatorname{col}\left(0,0, P_{0}\right)$ and $\left.\phi_{2 B}=0 ; 2\right)$ with the proposed stochastic boundary controls; and 3) with deterministic boundary controls for comparison. The simulation time is 200 seconds and motions at $s=50 i \mathrm{~m}, i=1, \ldots, 10$ are examined. The angles $\theta_{i}, i=1,2,3$ are calculated from $q_{i}, i=$ $1, \ldots, 4$, see [34]. The initial conditions are taken as $t_{0}=$ $0 ; \tilde{\boldsymbol{r}}_{10}(s)=\operatorname{col}(10 \sin (s / 10), 10 \cos (s / 10), 2 \sin (s / 15))$; $\boldsymbol{\theta}_{0}(s)=\operatorname{col}(\sin (s / 15), \cos (s / 15), 0.5 \sin (s / 15)) ;$ and all other initial values are chosen to be zero. Results for each simulation are detailed in what follows.

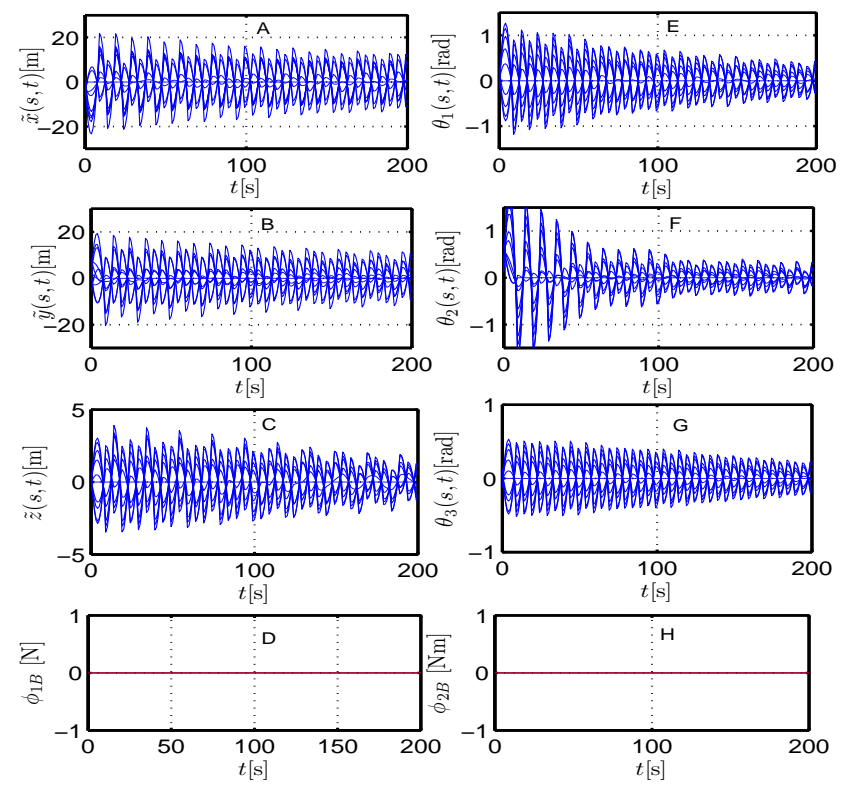

Fig. 2. Results with only constant tension $P_{0}$.

Case 1: For the case where only $P_{0}$ is applied, the results are plotted in Fig. 2. The displacements $(\tilde{x}(s, t), \tilde{y}(s, t), \tilde{z}(s, t))$ are plotted in Sub-figs. 2.A, 2.B and $2 . \mathrm{C}$, the rotations $\left(\theta_{1}(s, t), \theta_{2}(s, t), \theta_{3}(s, t)\right)$ are plotted in Sub-figs. 2.E, 2.F and 2.G at the aforementioned locations. The displacements and rotations oscillate with quite large magnitudes due to the sea loads but are bounded due to the tension $P_{0}$, which provides structural stiffness. The magnitude of $\tilde{z}(s, t)$ is much smaller than $\tilde{x}(s, t)$ and $\tilde{y}(s, t)$ because the beam is slender. All the motions seem to converge at a very slow rate mainly due to the damping. The controls are plotted in Sub-figs. 2.D and 2.H with $\phi_{1 B}^{*}:=\phi_{1 B}-\operatorname{col}\left(0,0, P_{0}\right)$.

Case 2: For the case with the proposed stochastic boundary control, the control gains $\boldsymbol{C}_{1 B}, \boldsymbol{C}_{2 B}, \gamma$, and the constant axial $P_{0}$ are chosen as $\boldsymbol{C}_{1 B}=\frac{1}{20} E A \boldsymbol{I}_{3}$,
$\boldsymbol{C}_{2 B}=\frac{1}{15} E I \boldsymbol{I}_{3}, \gamma=\frac{1}{6 \Gamma}$, and $P_{0}=1.8 E A$. It is readily checked that the above choice of the control gains and the constant axial force $P_{0}$ ensure that all the conditions (49) and (70) for some positive constants $\epsilon_{0 i}, i=1, \ldots, 5 ; \epsilon_{1 i}, i=1, \ldots, 12 ; \epsilon_{2 i}, i=1, \ldots, 9$; $\delta_{i B}, i=1,2 ; \varrho_{i B}, i=1,2$. The results are plotted in Fig.3. Comparing Sub-figs. (3.A, 3.B, 3.C) and (3.E, 3.F, 3.G) in this case with corresponding Sub-figs. (2.A, 2.B, 2.C) and (2.E, 2.F, 2.G) in the case where only $P_{0}$ is applied clearly shows that a significant reduction (about 20 times less) in magnitude of all displacements and rotations at all the examined locations. Note that this simulation was carried out for an extreme case, i.e., the riser outer diameter of $0.61 \mathrm{~m}$, inner diameter of $0.41 \mathrm{~m}$, length of $500 \mathrm{~m}$, and under very large sea loads, see the parameters used in $(75),(76),(77)$. This results in a quite large magnitude of $\phi_{1 B}$ and $\phi_{2 B}$ as shown in Sub-figs. 3.D and 3.H.
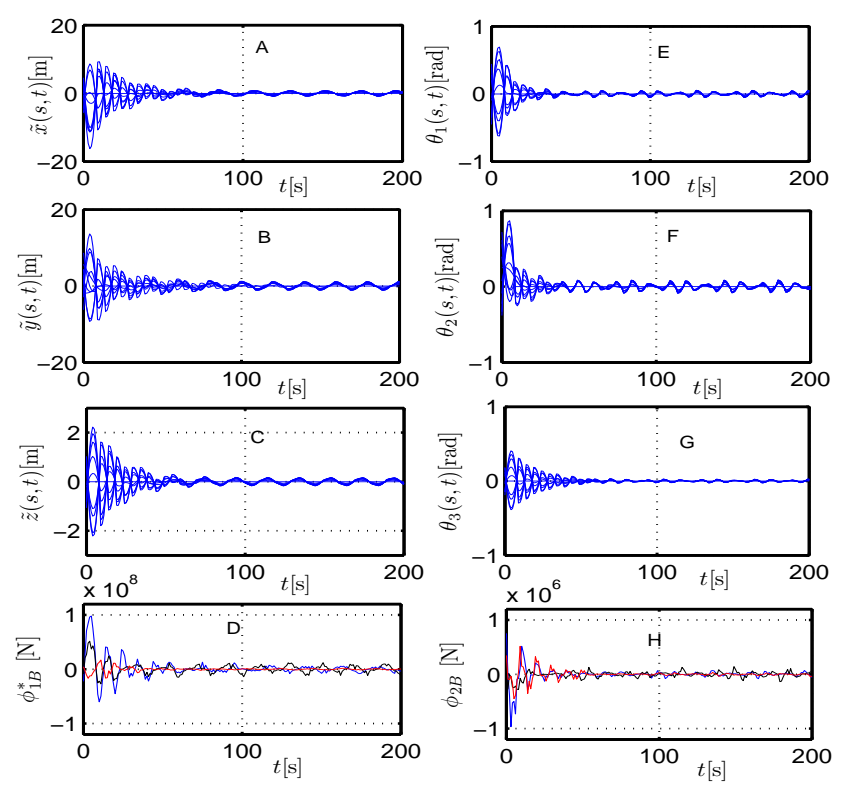

Fig. 3. Results with proposed boundary controls.

Case 3: Since stochastic boundary control of slender beams with all large motions in 3D has not been considered, we here provide a simulation result from deterministic control design for a comparison. A deterministic design (e.g., [9]) would result in boundary controls similar to (63) but the control gains need to satisfy less restrictive conditions, see [9] due to neglecting stochastic disturbances. To make a fair comparison, we impose a unit saturation on $\frac{d \boldsymbol{W}_{i}}{d t}$ and $\frac{d \boldsymbol{W}_{i B}}{d t}, i=1,2$ in (11) and (17) since these are unbounded in the deterministic sense. The control gains and $P_{0}$ are chosen as $\boldsymbol{C}_{1 B}=\frac{1}{15} E A \boldsymbol{I}_{3}$, $\boldsymbol{C}_{2 B}=\frac{1}{10} E I \boldsymbol{I}_{3}, \gamma=\frac{1}{4 \Gamma}$, and $P_{0}=2 E A$, see [9]. Note that these gains are allowed to be larger than Case 2 . The results are plotted in Fig. 4. Comparing Fig. 2 with Fig. 4 shows that there is a reduction in magnitude of all motions. This illustrates effectiveness of a deterministic control design. However, comparing Fig.3 with Fig.4 
shows that the stochastic control design gives a significant reduction. Note also from Sub-figs. 3.D, 3.H; and 4.D, 4.H that the deterministic control design results in quite larger control efforts in spite of a small increase in $\ell$. This is because a small increase in $\gamma$ results in a large decrease in $c_{0}$. This makes states converge to a larger ball and hence larger control efforts. Indeed, if $\frac{d \boldsymbol{W}_{i}}{d t}$ and $\frac{d \boldsymbol{W}_{i B}}{d t}, i=1,2$ in (11) and (17) are saturated at a higher magnitude, the control performance is worse.
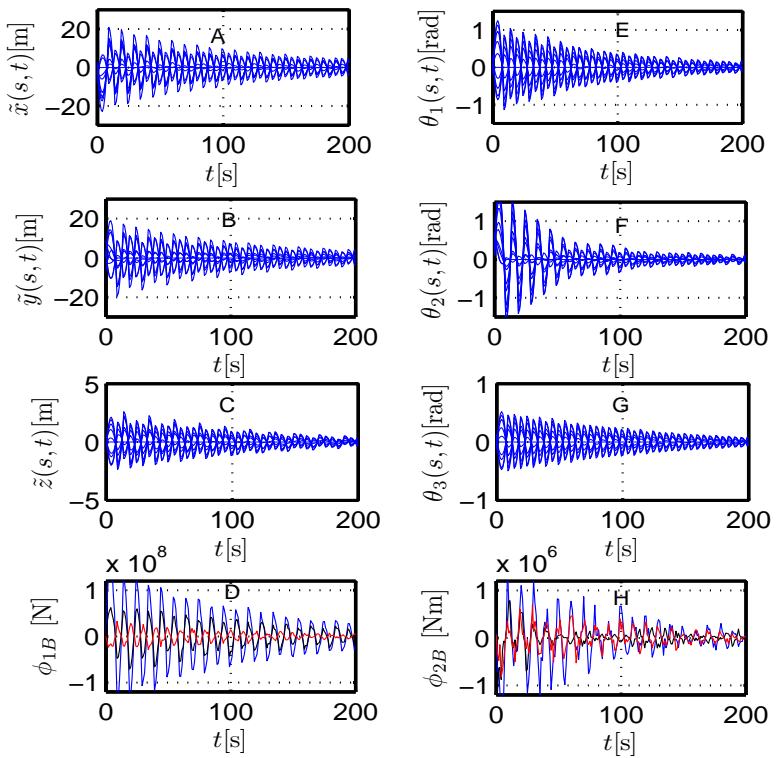

Fig. 4. Results with deterministic boundary controls in [9].

\section{Conclusions}

Nonlinear PDEs were derived to describe motions of the extensible and shearable slender beams with large deformations and rotations in space under both deterministic and stochastic loads induced by flows. Using Lyapunov's direct method, boundary controllers were designed to achieve global practical exponential $p$-stability of the resulting closed-loop system. Future work is to consider beams with a curved reference configuration in space.

\section{Appendices}

\section{A Proof of Theorem 4.1}

\section{A.1 Preparing Lemma}

We first prove a lemma that requires global monotonicity and growth conditions. Then, it is extended to the case where only local monotonicity and growth conditions are needed in Subsection A.2.

Lemma A.1 Suppose that Assumption 4.1 holds for any $\varepsilon$, i.e., the local conditions (31) and (32) are replaced by global ones. Then the condition of Item 1) of Theorem 4.1 ensures global existence and uniqueness of the variational strong solution of (25).
Proof of existence. Let $\phi_{k}, k=1,2, \ldots$ be orthonormal basis of $H$ obtained by the Gramm-Schmidt orthonormalization process from a dense linearly independent subset of $V$. Define $\boldsymbol{P}_{n}: V^{*} \rightarrow H_{n}=$ $\operatorname{span}\left\{\phi_{1}, \phi_{2}, \ldots, \phi_{n}\right\}$ by

$$
\boldsymbol{P}_{n} \boldsymbol{v}=\sum_{i=1}^{n}\left\langle\phi_{i}, \boldsymbol{v}\right\rangle_{V, V^{*}} \phi_{i}, \boldsymbol{v} \in V^{*} .
$$

Then $\boldsymbol{P}_{n} \mid H$ is just the orthogonal projection onto $H_{n}$ in $H$, and note that $H_{n}$ is a finite dimensional space. Moreover, we have

$$
\left\langle\boldsymbol{u}, \boldsymbol{P}_{n} \boldsymbol{F}(\boldsymbol{v}, t)\right\rangle_{V, V^{*}}=\langle\boldsymbol{u}, \boldsymbol{F}(\boldsymbol{v}, t)\rangle_{V, V^{*}}
$$

for all $\boldsymbol{v} \in V, \boldsymbol{u} \in H_{n}$. By Hypothesis H0 in Assumption 4.1 , for $\boldsymbol{u} \in H_{n}$ we have

$$
\left\langle\phi_{i}, \boldsymbol{u}\right\rangle_{V}=0, i \geq n+1 .
$$

By (33) and (34), for $\boldsymbol{v} \in H_{n}$ we have $\boldsymbol{F}(\boldsymbol{v}, t) \in V$ and $\sum_{i=1}^{\infty}\left\langle\phi_{i}, \boldsymbol{F}(\boldsymbol{v}, t)\right\rangle_{V, V^{*}} \phi_{i}=\sum_{i=1}^{\infty}\left\langle\phi_{i}, \boldsymbol{F}(\boldsymbol{v}, t)\right\rangle_{H} \phi_{i}=\boldsymbol{F}(\boldsymbol{v}, t)$.

Then for any $\boldsymbol{u}, \boldsymbol{v} \in H_{n}$, we have

$$
\begin{aligned}
\left\langle\boldsymbol{u}, \boldsymbol{P}_{n} \boldsymbol{F}(\boldsymbol{v}, t)\right\rangle_{V} & =\left\langle\boldsymbol{u}, \sum_{i=1}^{n}\left\langle\phi_{i}, \boldsymbol{F}(\boldsymbol{v}, t)\right\rangle_{V, V^{*}} \phi_{i}\right\rangle_{V} \\
& =\langle\boldsymbol{u}, \boldsymbol{F}(\boldsymbol{v}, t)\rangle_{V} .
\end{aligned}
$$

Let $\boldsymbol{W}_{n}(t):=\sum_{k=1}^{n} \sqrt{\lambda_{k}} \beta_{k}(t) e_{k}=\widetilde{\boldsymbol{P}}_{n} \boldsymbol{W}(t)$, where $\widetilde{\boldsymbol{P}}_{n}$ is the orthogonal projection from $\boldsymbol{K}_{0}$ onto $\operatorname{span}\left(e_{1}, \ldots, e_{n}\right)$. For each $n$, we consider the approximate system on $H_{n}$

$$
\begin{aligned}
& d \boldsymbol{X}_{n}=\boldsymbol{P}_{n} \boldsymbol{F}\left(\boldsymbol{X}_{n}, t\right) d t+\boldsymbol{P}_{n} \boldsymbol{G}\left(\boldsymbol{X}_{n}, t\right) d \boldsymbol{W}_{n}(t), \\
& \boldsymbol{X}_{n}\left(t_{0}\right)=\boldsymbol{P}_{n} \boldsymbol{X}_{0},
\end{aligned}
$$

which under conditions of Lemma A.1, see [21,30], has a unique solution $\boldsymbol{X}_{n}(t) \in H_{n}$ satisfying

$$
\begin{aligned}
\boldsymbol{X}_{n}(t)= & \boldsymbol{P}_{n} \boldsymbol{X}_{0}+\int_{t_{0}}^{t} \boldsymbol{P}_{n} \boldsymbol{F}\left(\boldsymbol{X}_{n}(s), s\right) d s \\
& +\int_{t_{0}}^{t} \boldsymbol{P}_{n} \boldsymbol{G}\left(\boldsymbol{X}_{n}(s), s\right) d \boldsymbol{W}_{n}(s) d s .
\end{aligned}
$$

Identifying $\boldsymbol{X}_{n}(t)$ with an $\mathbb{R}^{n}$-valued process and applying the Itô formula to $U\left(\boldsymbol{X}_{n}(t), t\right)$ yields

$U\left(\boldsymbol{X}_{n}(t), t\right)=U\left(\boldsymbol{X}_{n}\left(t_{0}\right), t_{0}\right)+\int_{t_{0}}^{t} \mathcal{L} U\left(\boldsymbol{X}_{n}(\sigma), \sigma\right) d \sigma+M_{n}(t)$,

where $\mathcal{L} U\left(\boldsymbol{X}_{n}(\sigma), \sigma\right)$ is obtained by applying (29) to (A.7), and

$$
M_{n}(t)=\int_{t_{0}}^{t}\left\langle U_{\boldsymbol{X}_{n}}\left(\boldsymbol{X}_{n}(\sigma), \sigma\right), \boldsymbol{P}_{n} \boldsymbol{G}_{n}\left(\boldsymbol{X}_{k}(\sigma), \sigma\right) d \boldsymbol{W}_{n}(\sigma)\right\rangle_{V}
$$

Let $\hat{\eta}_{l}:=\inf \left\{\sigma_{l} \geq t_{0}:\left\|\boldsymbol{X}_{n}\left(\sigma_{l}\right)\right\|_{V}>l\right\} \wedge t, l=$ $1,2, \ldots$ Since $\boldsymbol{X}_{n}(\sigma), t_{0} \leq \sigma \leq t$ is right continuous and $\mathcal{F}$-adapted, $\sigma_{l}$ is a stopping time for every $l \in \mathbb{N}$. Thus, $\exists \eta_{l}$ such that $\eta_{l}=\inf \left\{U\left(\boldsymbol{X}_{n}(t), t\right) \geq l\right\}$ because of (33). Thus, $\tau_{l}=\sigma_{l} \wedge \eta_{l}$ localizes $M_{n}, \tau_{l} \rightarrow \infty$. Therefore, taking expectation of (A.8) and using (34) yields

$$
\begin{aligned}
\mathbb{E}\left\{U\left(\boldsymbol{X}_{n}\left(t \wedge \tau_{l}\right), t\right)\right\}=\mathbb{E}\left\{U\left(\boldsymbol{X}_{n}\left(t_{0}\right), t_{0}\right)\right\} \\
+\int_{t_{0}}^{t} \mathbb{E}\left\{\mathcal{I}_{\left[t_{0}, \tau_{l}\right]} \mathcal{L} U\left(\boldsymbol{X}_{n}(\sigma), \sigma\right) d \sigma\right\} .
\end{aligned}
$$


Letting $l \rightarrow \infty$ and using the condition (34) give

$$
\begin{aligned}
& \mathbb{E}\left\{U\left(\boldsymbol{X}_{n}(t), t\right)\right\} \leq \mathbb{E}\left\{U\left(\boldsymbol{X}_{n}\left(t_{0}\right), t_{0}\right)\right\} \\
& +\int_{t_{0}}^{t} \mathbb{E}\left\{c_{3}\left(1+U\left(\boldsymbol{X}_{n}(\sigma), \sigma\right)\right)\right\} d \sigma
\end{aligned}
$$

Applying Gronwall's inequality, see [1], to (A.11) yields $\mathbb{E}\left\{U\left(\boldsymbol{X}_{n}(t), t\right)\right\} \leq\left(c_{3}\left(t-t_{0}\right)+\mathbb{E}\left\{U\left(\boldsymbol{X}_{n}\left(t_{0}\right), t_{0}\right)\right\}\right) e^{c_{3}\left(t-t_{0}\right)}$,

which implies from the condition (34) that

$$
\begin{aligned}
\mathbb{E}\left\{\left\|\boldsymbol{X}_{n}(t)\right\|_{V}^{p}\right\} & \leq \alpha_{1}^{-1}\left[\left(c_{3}\left(t-t_{0}\right)\right.\right. \\
& \left.\left.+\mathbb{E}\left\{\alpha_{2}\left(\left\|\boldsymbol{X}_{n}\left(t_{0}\right)\right\|_{V}^{p}\right)\right\}\right) e^{c_{3}\left(t-t_{0}\right)}\right] .
\end{aligned}
$$

Hence, $\boldsymbol{X}_{n}(t)$ is bounded in $L^{2}\left(\left[t_{0}, \infty\right) \times \Omega, V\right)$ since $p \geq 2$ and $\alpha_{1}^{-1}$ is also a class $\mathcal{K}_{\infty}$-function. The condition $(3 \overline{4})$ together boundedness of $\boldsymbol{X}_{n}(t)$ in $L^{2}\left(\left[t_{0}, \infty\right) \times \Omega, V\right)$ ensures that $\boldsymbol{X}_{n}(t)$ is bounded in $L^{2}\left(\left[t_{0}, \infty\right) \times \Omega, H\right)$. Using (34), we also have boundedness of $\boldsymbol{F}\left(\boldsymbol{X}_{n}(),.\right)$ in $L^{2}\left(\left[t_{0}, \infty\right) \times \Omega, V^{*}\right)=\left(L^{2}\left(\left[t_{0}, \infty\right) \times \Omega, V\right)\right)^{*}$, $\boldsymbol{P}_{n} \boldsymbol{G}\left(\boldsymbol{X}_{n}(),.\right)$ in $L^{2}\left(\left[t_{0}, \infty\right) \times \Omega, \mathscr{L}_{2}^{0}\right)$. Therefore, by the Alaoglu theorem and passing to a subsequence if needed, we can assume that there exist $\boldsymbol{X}, \boldsymbol{Y}$, and $\boldsymbol{Z}$ such that

$$
\begin{array}{r}
\boldsymbol{X}_{n} \rightarrow \boldsymbol{X} \text { weakly in } L^{2}\left(\left[t_{0}, \infty\right) \times \Omega, V\right) \text { and weakly } \\
\text { star in } L^{2}\left(\left[t_{0}, \infty\right) \times \Omega, L^{\infty}\left(\left[t_{0}, \infty\right), V\right),\right. \\
\boldsymbol{P}_{n} \boldsymbol{F}\left(\boldsymbol{X}_{n}(.), .\right) \rightarrow \boldsymbol{Y} \text { weakly in } L^{2}\left(\left[t_{0}, \infty\right) \times \Omega, V^{*}\right), \\
\boldsymbol{P}_{n} \boldsymbol{G}\left(\boldsymbol{X}_{n}(.), .\right) \rightarrow \boldsymbol{Z} \text { weakly in } L^{2}\left(\left[t_{0}, \infty\right) \times \Omega, \mathscr{L}_{2}^{0}\right) .
\end{array}
$$

Then, it is a routine to show that $\boldsymbol{Y}(t)=\boldsymbol{F}(\boldsymbol{X}(t), t)$ and $\boldsymbol{Z}(t)=\boldsymbol{G}(\boldsymbol{X}(t), t), d t \otimes d \mathbb{P}$-a.e, and uniqueness of the solution, see [12].

\section{A.2 Proof of Item 1) of Theorem 4.1}

Let $k_{0}$ be the upperbound of the initial data, i.e., $\left\|\boldsymbol{X}_{0}\right\|_{V} \leq k_{0}$. For any integer $k>k_{0}$, let us define

$\boldsymbol{F}_{k}(\boldsymbol{X}, t)=\boldsymbol{F}\left(\frac{\|\boldsymbol{X}\|_{V} \wedge k}{\|\boldsymbol{X}\|_{V}} \boldsymbol{X}, t\right), \boldsymbol{G}_{k}(\boldsymbol{X}, t)=\boldsymbol{G}\left(\frac{\|\boldsymbol{X}\|_{V} \wedge k}{\|\boldsymbol{X}\|_{V}} \boldsymbol{X}, t\right)$,

where we let $\frac{\|\boldsymbol{X}\|_{V} \wedge k}{\|\boldsymbol{X}\|_{V}} \boldsymbol{X}=0$ if $\boldsymbol{X}=0$. Then, all the conditions of Lemma A.1 hold for all $\boldsymbol{X} \in H$ with $\|\boldsymbol{X}\|_{V} \leq$ $\varepsilon, \varepsilon>0$. Thus, Lemma A.1 ensures well-posedness of the variational solution $\boldsymbol{X}_{k}(t)$ in $L^{p}\left(\Omega ; \mathcal{C}\left(\left[t_{0}, T\right] ; V\right)\right)$ for arbitrarily $T>t_{0}$ to the SES:

$$
d \boldsymbol{X}_{k}=\boldsymbol{F}_{k}\left(\boldsymbol{X}_{k}, t\right) d t+\boldsymbol{G}_{k}\left(\boldsymbol{X}_{k}, t\right) d \boldsymbol{W}(t)
$$

for any $\boldsymbol{X}_{k}\left(t_{0}\right)=\boldsymbol{X}_{0} \in V$. Define a stopping time

$$
s_{k}=\inf \left\{t \geq t_{0}:\left\|\boldsymbol{X}_{k}(t)\right\|_{V}>k\right\},
$$

where we let $\inf \emptyset=\infty$. Obviously for any $\sigma<s_{k}$, we have $\left\|\boldsymbol{X}_{k}(\sigma)\right\|_{V} \leq k$. From (A.15), it is seen that

$$
\begin{aligned}
& \boldsymbol{F}_{k+1}\left(\boldsymbol{X}_{k}(\sigma), t\right)=\boldsymbol{F}_{k}\left(\boldsymbol{X}_{k}(\sigma), t\right), \\
& \boldsymbol{G}_{k+1}\left(\boldsymbol{X}_{k}(\sigma), t\right)=\boldsymbol{G}_{k}\left(\boldsymbol{X}_{k}(\sigma), t\right),
\end{aligned}
$$

$\forall t_{0} \leq \sigma \leq s_{k}$. Using (A.18) and Itô's formula, we have

$$
\boldsymbol{X}_{k+1}(t)=\boldsymbol{X}_{k}(t), \quad \forall t_{0} \leq t \leq s_{k},
$$

and that $s_{k}$ is increasing in $k$. This means that we can define $s=\lim _{k \rightarrow \infty} s_{k}$. From (A.19), we also have

$$
\boldsymbol{X}(t)=\boldsymbol{X}_{k}(t), \quad \forall t_{0} \leq t<s_{k} .
$$

This together with (A.18) means that $\boldsymbol{X}(t)$ is the unique strong solution of (25) for $t \in\left[t_{0}, s_{k}\right)$. Then, it is a routine to show that $\mathbb{P}(s=\infty)=1$, see [12].

\section{A.3 Proof of Item 2) of Theorem 4.1}

Applying the Itô formula to $e^{c_{4}\left(t-t_{0}\right)} U(\boldsymbol{X}, t)$ and the solution $\boldsymbol{X}(t)$ of (25), and using (35) give

$$
\begin{aligned}
& e^{c_{4}\left(t-t_{0}\right)} U(\boldsymbol{X}(t), t) \leq U\left(\boldsymbol{X}\left(t_{0}\right), t_{0}\right)+c_{0} \int_{t_{0}}^{t} e^{c_{4}\left(r-t_{0}\right)} d r \\
& \quad+\int_{t_{0}}^{t}\left\langle U_{\boldsymbol{X}}(\boldsymbol{X}(r), r), \boldsymbol{G}(\boldsymbol{X}(r), r) d \boldsymbol{W}(r)\right\rangle_{H},
\end{aligned}
$$

Since the last integral in (A.21) is a martingale, taking expectancy of (A.21), using (33), and using the Sobolev embedding (i.e., $V \subset H)$ give (36) .

\section{B Calculation of $\mathcal{L} U_{0}$ and $\mathcal{L} U_{1}$}

\section{B.1 Calculation of $\mathcal{L} U_{0}$}

Applying the Itô formula to $U_{0}$ given in (41) gives

$$
\mathcal{L} U_{0}=\mathcal{L} U_{01}+\mathcal{L} U_{02} .
$$

Applying Itô's formula to $U_{01}$ results in

$$
\mathcal{L} U_{01}=\sum_{i=1}^{5} A_{01 i},
$$

where

$$
\begin{aligned}
& A_{011}=-\langle\boldsymbol{v}, \boldsymbol{\omega} \times(\boldsymbol{M} \boldsymbol{v})\rangle_{L^{2}}+\left\langle\boldsymbol{\omega}, \boldsymbol{v} \times\left(\boldsymbol{M}_{A} \boldsymbol{v}\right)\right\rangle_{L^{2}} \\
& \quad-\langle\boldsymbol{\omega}, \boldsymbol{\omega} \times(\boldsymbol{J} \boldsymbol{\omega})\rangle_{L^{2}}, \\
& A_{012}=\left\langle\boldsymbol{v}, \boldsymbol{D}_{A 11} \boldsymbol{v}+\boldsymbol{D}_{A 12} \boldsymbol{v} \otimes \boldsymbol{v} \boldsymbol{v}\right\rangle_{L^{2}} \\
& \quad+\left\langle\boldsymbol{\omega}, \boldsymbol{D}_{A 21} \boldsymbol{\omega}+\boldsymbol{D}_{A 22} \boldsymbol{\omega} \otimes \boldsymbol{\omega} \boldsymbol{\omega}\right\rangle_{L^{2}}, \\
& A_{013}=\left\langle\boldsymbol{v}, \boldsymbol{R}_{1}^{-1}(\boldsymbol{q}) \mathbb{D} \boldsymbol{n}\right\rangle_{L^{2}}+\langle\boldsymbol{\omega}, \mathbb{D} \boldsymbol{m}\rangle_{L^{2}}, \\
& A_{014}=\left\langle\boldsymbol{v}, \boldsymbol{f}_{1}\right\rangle_{L^{2}}+\left\langle\boldsymbol{\omega}, \boldsymbol{f}_{2}\right\rangle_{L^{2}}, \\
& A_{015}=\frac{1}{2} \operatorname{Tr}\left(\left(\boldsymbol{G}_{1} \boldsymbol{\Delta}_{1}\right)\left(\boldsymbol{G}_{1} \boldsymbol{\Delta}_{1}\right)^{T}\right)+\frac{1}{2} \operatorname{Tr}\left(\left(\boldsymbol{G}_{2} \boldsymbol{\Delta}_{2}\right)\left(\boldsymbol{G}_{2} \boldsymbol{\Delta}_{2}\right)^{T}\right) .
\end{aligned}
$$

The term $A_{011}$ : Since $\boldsymbol{M}=\boldsymbol{I}_{3} m_{0}+\boldsymbol{M}_{A}$, we have

$$
\begin{gathered}
A_{011}=-\left\langle\boldsymbol{v}, \boldsymbol{\omega} \times\left(\boldsymbol{I}_{3} m_{0} \boldsymbol{v}\right)\right\rangle_{L^{2}}-\left\langle\boldsymbol{v}, \boldsymbol{\omega} \times\left(\boldsymbol{M}_{A} \boldsymbol{v}\right)\right\rangle_{L^{2}} \\
+\left\langle\boldsymbol{\omega}, \boldsymbol{v} \times\left(\boldsymbol{M}_{A} \boldsymbol{v}\right)\right\rangle_{L^{2}}-\langle\boldsymbol{\omega}, \boldsymbol{\omega} \times(\boldsymbol{J} \boldsymbol{\omega})\rangle_{L^{2}}=0 .
\end{gathered}
$$

The term $A_{012}$ : Using Assumption 3.1, we have

$$
\begin{aligned}
A_{012} & \leq-d_{A 11}^{m}\|\boldsymbol{v}\|_{L^{2}}^{2}-d_{A 12}^{m}\left(\left\|v_{1}^{2}\right\|_{L^{2}}^{2}+\left\|v_{2}^{2}\right\|_{L^{2}}^{2}+\left\|v_{3}^{2}\right\|_{L^{2}}^{2}\right) \\
& -d_{A 21}^{m}\|\boldsymbol{\omega}\|_{L^{2}}^{2}-d_{A 22}^{m}\left(\left\|\omega_{1}^{2}\right\|_{L^{2}}^{2}+\left\|\omega_{2}^{2}\right\|_{L^{2}}^{2}+\left\|\omega_{3}^{2}\right\|_{L^{2}}^{2}\right) .
\end{aligned}
$$

where $v_{i}$ and $\omega_{i}$ are the $i$ elements of $\boldsymbol{v}$ and $\boldsymbol{\omega}$.

The term $A_{013}$ : Using integration by parts and the fact that $\mathbb{D} \boldsymbol{n}=\mathbb{D}\left(\boldsymbol{n}-P_{0} \boldsymbol{e}_{3}\right)$, we have

$$
\begin{aligned}
& A_{013}=\left(\boldsymbol{n}^{B \Gamma}-P_{0} \boldsymbol{e}_{3}\right)^{T} \tilde{\boldsymbol{r}}_{t}^{B \Gamma}+\left(\boldsymbol{m}^{B \Gamma}\right)^{T} \boldsymbol{\omega}^{B \Gamma} \\
& \quad-\sum_{i=1}^{2} G \bar{A}_{i}\left\langle\eta_{i t},\left(\eta_{i}-\frac{1}{2} \eta_{i}^{2}+\frac{1}{3} \eta_{i}^{3}\right)\right\rangle_{L^{2}} \\
& \quad-E A\left\langle\varepsilon_{t},\left(\varepsilon-\frac{1}{2} \varepsilon^{2}+\frac{1}{3} \varepsilon^{3}\right)\right\rangle_{L^{2}} \\
& \quad-\sum_{i=1}^{2} E I_{i}\left\langle\mu_{i t},\left(\mu_{i}-\frac{1}{2} \mu_{i}^{2}+\frac{1}{3} \mu_{i}^{3}\right)\right\rangle_{L^{2}} \\
& \quad-G I_{3}\left\langle\mu_{3 t},\left(\mu_{3}-\frac{1}{2} \mu_{3}^{2}+\frac{1}{3} \mu_{3}^{3}\right)\right\rangle_{L^{2}}-P_{0}\left\langle 1, \varepsilon_{t}-\mathbb{D} \tilde{z}_{t}\right\rangle_{L^{2}},
\end{aligned}
$$


where we have used (15) and the fifth equation of (24), $P_{0}\left\langle\boldsymbol{e}_{3}, \mathbb{D} \tilde{\boldsymbol{r}}_{t}\right\rangle_{L^{2}}=P_{0}\left\langle 1, \mathbb{D} \tilde{z}_{t}\right\rangle_{L^{2}}, \varepsilon_{t}:=\frac{\partial \varepsilon}{\partial t}, \eta_{i t}:=\frac{\partial \eta_{i}}{\partial t}$, $\mu_{i t}:=\frac{\partial \mu_{i}}{\partial t}$, and $\tilde{z}_{t}:=\frac{\partial \tilde{z}}{\partial t}$.

The term $A_{014}$ : Substituting $\boldsymbol{f}_{1}$ and $\boldsymbol{f}_{2}$ in (12) into $A_{014}$ in (B.3) and using $\left\langle\boldsymbol{v}, \boldsymbol{\omega} \times\left(\boldsymbol{M}_{A} \overline{\boldsymbol{v}}_{f}\right)\right\rangle_{L^{2}}+\langle\boldsymbol{\omega}, \boldsymbol{v} \times$ $\left.\left(\boldsymbol{M}_{A} \overline{\boldsymbol{v}}_{f}\right)\right\rangle_{L^{2}}=0$ and $\left\langle\boldsymbol{\omega}, \boldsymbol{\omega} \times\left(\boldsymbol{J}_{A} \overline{\boldsymbol{\omega}}_{f}\right)\right\rangle_{L^{2}}=0$ yields

$$
\begin{aligned}
A_{014} & \leq \lambda_{M}\left(\left|\boldsymbol{M}_{A}\right|\right) \bar{\omega}_{f}^{M}\|\boldsymbol{v}\|_{L^{2}}^{2}+3 d_{A 12}^{M} \bar{v}_{f}^{M} \epsilon_{01}\|\boldsymbol{v}\|_{L^{2}}^{2} \\
& +\frac{3 d_{A 12}^{M}}{4 \epsilon_{01}}\left\||\boldsymbol{v}|^{2}\right\|_{L^{2}}+3 d_{A 12}\left(\bar{v}_{f}^{M}\right)^{2}\|\boldsymbol{v}\|_{L^{2}}^{2}+\epsilon_{02}\|\boldsymbol{v}\|_{L^{2}}^{2} \\
& +\frac{1}{4 \epsilon_{02}}\left\|\overline{\boldsymbol{f}}_{10}\right\|_{L^{2}}^{2}+\lambda_{M}\left(\left|\boldsymbol{J}_{A}\right|\right) \bar{\omega}_{f}^{M}\|\boldsymbol{\omega}\|_{L^{2}}^{2} \\
& +\lambda_{M}\left(\left|\boldsymbol{M}_{A}\right|\right) \bar{v}_{f}^{M} \epsilon_{03}\|\boldsymbol{v}\|_{L^{2}}^{2}+\frac{\lambda_{M}\left(\left|\boldsymbol{M}_{A}\right|\right) \bar{v}_{f}^{M}}{4 \epsilon_{03}}\|\boldsymbol{\omega}\|_{L^{2}}^{2} \\
& +3 d_{A 21} \bar{\omega}_{f}^{M} \epsilon_{04}\|\boldsymbol{\omega}\|_{L^{2}}^{2}+\epsilon_{05}\|\boldsymbol{\omega}\|_{L^{2}}^{2}+\frac{1}{4 \epsilon_{05}}\left\|\overline{\boldsymbol{f}}_{20}\right\|_{L^{2}}^{2} \\
& +\frac{3 d_{A 22} \bar{\omega}_{f}^{M}}{4 \epsilon_{04}}\left\||\boldsymbol{\omega}|^{2}\right\|_{L^{2}}^{2}+3 d_{A 22}^{M}\left(\bar{\omega}_{f}^{M}\right)^{2}\|\boldsymbol{\omega}\|_{L^{2}}^{2},
\end{aligned}
$$

where $\epsilon_{0 i}, i=1, \ldots, 5$ are positive constants, $|\bullet|$ denotes

- with its elements replaced by their absolute values.

The term $A_{015}$ : Substituting $\boldsymbol{G}_{1}$ and $\boldsymbol{G}_{2}$ in (12) and $\boldsymbol{\Delta}_{1}$ and $\boldsymbol{\Delta}_{2}$ into $A_{015}$ in (B.3) yields

$$
\begin{aligned}
A_{015} \leq & 3 \Sigma_{i=1}^{6} \operatorname{Tr}\left(\left(\boldsymbol{G}_{1 i} \boldsymbol{\Delta}_{1 i}\right)\left(\boldsymbol{G}_{1 i} \boldsymbol{\Delta}_{1 i}\right)^{T}\right) \\
& +4 \Sigma_{i=1}^{8} \operatorname{Tr}\left(\left(\boldsymbol{G}_{2 i} \boldsymbol{\Delta}_{2 i}\right)\left(\boldsymbol{G}_{2 i} \boldsymbol{\Delta}_{2 i}\right)^{T}\right) .
\end{aligned}
$$

Substituting $\boldsymbol{G}_{1 i}$ and $\boldsymbol{G}_{2 i}$ defined in (13) into (B.8) and applying Young's and Hölder's inequalities, we have

$$
\begin{aligned}
& A_{015} \leq 9 \lambda_{M}^{2}\left(\left|\boldsymbol{M}_{A}\right|\right) \delta_{11}^{M}\|\boldsymbol{\omega}\|_{L^{2}}^{2}+9 \lambda_{M}^{2}\left(\left|\boldsymbol{M}_{A}\right|\right) \delta_{12}^{M}\|\boldsymbol{v}\|_{L^{2}}^{2} \\
& \quad+27 d_{A 12}^{M}\left(\delta_{13}^{M}\right)^{2}\|\boldsymbol{v}\|_{L^{2}}^{2}+\left.108\left(d_{A 12}^{M}\right)^{2}\left(\delta_{14}^{M}\right)^{2}\|\| \boldsymbol{v}\right|^{2} \|_{L^{2}}^{2} \\
& \quad+3\left(\delta_{16}^{M}\right)^{2}+12 \lambda_{M}^{2}\left(\left|\boldsymbol{J}_{A}\right|\right)\|\boldsymbol{\omega}\|_{L^{2}}^{2}\left(\left(\delta_{21}^{M}\right)^{2}+\left(\delta_{22}^{M}\right)^{2}\right) \\
& \quad+12\left[\lambda_{M}^{2}\left(\left|\boldsymbol{M}_{A}\right|\right)\left(\delta_{23}^{M}\right)^{2}+\lambda_{M}^{2}\left(\left|\boldsymbol{J}_{A}\right|\right)\left(\delta_{24}^{M}\right)^{2}\right]\|\boldsymbol{v}\|_{L^{2}}^{2} \\
& +12\left(d_{A 22}^{M}\right)^{2}\left[\left(\delta_{25}^{M}\right)^{2}+2\left(\delta_{26}^{M}\right)^{2}\right]\|\boldsymbol{\omega}\|_{L^{2}}^{2} \\
& +12\left(d_{A 22}^{M}\right)^{2}\left(\delta_{27}^{M}\right)^{2}\left\||\boldsymbol{\omega}|^{2}\right\|_{L^{2}}^{2}+4\left(\delta_{28}^{M}\right)^{2} .
\end{aligned}
$$

Applying the Itô formula to $U_{02}$ results in

$$
\begin{aligned}
& \mathcal{L} U_{02}=\sum_{i=1}^{2} G \bar{A}_{i}\left\langle\eta_{i t},\left(\eta_{i}-\frac{1}{2} \eta_{i}^{2}+\frac{1}{3} \eta_{i}^{3}\right)\right\rangle_{L^{2}} \\
& \quad+E A\left\langle\varepsilon_{t},\left(\varepsilon-\frac{1}{2} \varepsilon^{2}+\frac{1}{3} \varepsilon^{3}\right)\right\rangle_{L^{2}} \\
& \quad+\sum_{i=1}^{2} E I_{i}\left\langle\mu_{i t},\left(\mu_{i}-\frac{1}{2} \mu_{i}^{2}+\frac{1}{3} \mu_{i}^{3}\right)\right\rangle_{L^{2}} \\
& \quad+G I_{3}\left\langle\mu_{3 t},\left(\mu_{3}-\frac{1}{2} \mu_{3}^{2}+\frac{1}{3} \mu_{3}^{3}\right)\right\rangle_{L^{2}}+P_{0}\left\langle 1, \varepsilon_{t}-\mathbb{D} \tilde{z}_{t}\right\rangle_{L^{2}} .
\end{aligned}
$$

Substituting (B.4)-(B.9) into (B.2), then together with (B.10) into (B.1) results in (52).

\section{B.2 Calculation of $\mathcal{L} U_{11}$ and $\mathcal{L} U_{12}$}

B.2.1 Calculation of $\mathcal{L} U_{11}$

Applying Itô's formula to $U_{11}$ yields

$$
\mathcal{L} U_{11}=\sum_{i=1}^{5} A_{11 i}
$$

where

$$
\begin{aligned}
& A_{111}=\gamma\left\langle\mathbb{D} \tilde{\boldsymbol{r}}_{t}, \boldsymbol{R}_{1}(\boldsymbol{q}) \boldsymbol{M} \boldsymbol{v} s\right\rangle_{L^{2}}, \\
& A_{112}=\gamma\left\langle\mathbb{D} \tilde{\boldsymbol{r}}, \boldsymbol{R}_{1 t}(\boldsymbol{q}) \boldsymbol{M} \boldsymbol{v} s{L^{2}}-\gamma m_{0}\left\langle\mathbb{D} \tilde{\boldsymbol{r}}, \boldsymbol{R}_{1 t}(\boldsymbol{q}) \boldsymbol{v} s\right\rangle_{L^{2}},\right. \\
& A_{113}=\gamma\langle\mathbb{D} \tilde{\boldsymbol{r}}, \mathbb{D} \boldsymbol{n} s\rangle_{L^{2}}, A_{115}=\gamma\left\langle\mathbb{D} \tilde{\boldsymbol{r}}, \boldsymbol{f}_{1} s\right\rangle_{L^{2}}, \\
& A_{114}=\gamma\left\langle\mathbb{D} \tilde{\boldsymbol{r}},\left(\boldsymbol{\omega} \times\left(\boldsymbol{M}_{A} \boldsymbol{v}\right)+\boldsymbol{D}_{A 11} \boldsymbol{v}+\boldsymbol{D}_{A 12} \boldsymbol{v} \otimes \boldsymbol{v} \boldsymbol{v}\right) s\right\rangle_{L^{2}} .
\end{aligned}
$$

and the first equation of (2) has been used. We now calculate the terms $A_{11 i}, i=1, \ldots, 5$.

The term $A_{111}$ : Using $\boldsymbol{v}=\boldsymbol{R}^{-1}(\boldsymbol{q}) \tilde{\boldsymbol{r}}=\boldsymbol{R}^{T}(\boldsymbol{q}) \tilde{\boldsymbol{r}}$, see (7), $\gamma\left\langle\tilde{\boldsymbol{r}}_{t}, \boldsymbol{R}_{1}(\boldsymbol{q}) \boldsymbol{M} \boldsymbol{R}_{1}^{T}(\boldsymbol{q}) \mathbb{D} \tilde{\boldsymbol{r}}_{t} s\right\rangle_{L^{2}}=A_{111},\left\|\mathbb{D} \boldsymbol{R}_{1}(\boldsymbol{q})\right\|^{2} \leq$ $16^{2}\|\mathbb{D} \boldsymbol{q}\|^{2} \leq 64\|\boldsymbol{\mu}\|^{2}, \mathbb{D} \boldsymbol{q}=\boldsymbol{K}(\boldsymbol{q}) \boldsymbol{\mu}$, and integration by parts, we have

$$
\begin{aligned}
& A_{111} \leq \frac{\gamma \Gamma \lambda_{M}(\boldsymbol{M})}{2}\left\|\tilde{\boldsymbol{r}}_{t}^{B \Gamma}\right\|^{2}-\frac{\gamma \lambda_{m}(\boldsymbol{M})}{2}\|\boldsymbol{v}\|_{L^{2}}^{2} \\
& \quad+16 \gamma \Gamma \lambda_{M}(\boldsymbol{M}) \epsilon_{11}\|\boldsymbol{\mu}\|_{L^{2}}^{2}+\frac{\gamma \Gamma \lambda_{M}(\boldsymbol{M})}{8 \epsilon_{11}}\left\|\left.\boldsymbol{v}\right|^{2}\right\|_{L^{2}}^{2}
\end{aligned}
$$

where $\epsilon_{11}$ is a positive constant to be determined.

The term $A_{112}$ : Using $\boldsymbol{M}=\boldsymbol{I}_{3} m_{0}+\boldsymbol{M}_{A}$, and Young's and Hölder's inequalities, and (3) and (11), we have:

$$
\begin{gathered}
A_{112} \leq \frac{16 \gamma \Gamma \lambda_{M}\left(\left|\boldsymbol{M}_{A}\right|\right)}{\epsilon_{12}}\left\||\boldsymbol{v}|^{2}\right\|_{L^{2}}^{2} \\
+\gamma \Gamma \lambda_{M}\left(\left|\boldsymbol{M}_{A}\right|\right) \epsilon_{12}\|\mathbb{D} \tilde{\boldsymbol{r}}\|_{L^{2}}^{2}
\end{gathered}
$$

where $\epsilon_{12}$ is a positive constant to be determined.

The term $A_{113}$ : We use the trick $\mathbb{D} \boldsymbol{n}=\mathbb{D}\left(\boldsymbol{n}+P_{0} \boldsymbol{e}_{3}\right)$, $\boldsymbol{r}=\boldsymbol{r}^{0}+\tilde{\boldsymbol{r}}$, and integration by parts to write $A_{113}$ as

$$
\begin{aligned}
& A_{113}=\gamma \Gamma\left(\boldsymbol{n}^{B \Gamma}-P_{0} \boldsymbol{e}_{3}\right) \mathbb{D} \tilde{\boldsymbol{r}}^{B \Gamma}-\gamma\left\langle\boldsymbol{n}, \mathbb{D} \boldsymbol{r}+\mathbb{D}^{2} \boldsymbol{r} s\right\rangle_{L^{2}} \\
& +\gamma\left\langle\boldsymbol{n}, \mathbb{D} \boldsymbol{r}^{0}+\mathbb{D}^{2} \boldsymbol{r}^{0} s\right\rangle_{L^{2}}+\gamma P_{0}\left\langle\boldsymbol{e}_{3}, \mathbb{D} \tilde{\boldsymbol{r}}+\mathbb{D}^{2} \tilde{\boldsymbol{r}} s\right\rangle_{L^{2}}
\end{aligned}
$$

We calculate $-\gamma\left\langle\boldsymbol{n}, \mathbb{D} \boldsymbol{r}+\mathbb{D}^{2} \boldsymbol{r} s\right\rangle_{L^{2}}$ in the local basis and $\gamma\left\langle\boldsymbol{n}, \mathbb{D} \boldsymbol{r}^{0}+\mathbb{D}^{2} \boldsymbol{r}^{0} s\right\rangle_{L^{2}}$ in the fixed basis. Using the seventh equation of (24), and $\boldsymbol{r}^{0}$ and $\boldsymbol{n}$ are expressed in the fixed basis as $\boldsymbol{r}^{0}=0 \boldsymbol{e}_{1}+0 \boldsymbol{e}_{2}+s \boldsymbol{e}_{3}$ and $\boldsymbol{n}=Q_{1} \boldsymbol{R}_{1} \boldsymbol{e}_{1}+$ $Q_{2} \boldsymbol{R}_{1} \boldsymbol{e}_{2}+N \boldsymbol{R}_{1} \boldsymbol{e}_{3}$, we have

$$
\begin{aligned}
& A_{113} \leq \gamma \Gamma\left(\boldsymbol{n}^{B \Gamma}-P_{0} \boldsymbol{e}_{3}\right) \mathbb{D} \tilde{\boldsymbol{r}}^{B \Gamma}-\frac{5 \gamma \Gamma}{12}\left[G \bar{A}_{1} \wedge G \bar{A}_{2} \wedge E A\right] \\
& \times\left\|\boldsymbol{\vartheta}^{B \Gamma}\right\|^{2}-\frac{5 \gamma G \bar{A}_{1}}{12}\left\|\eta_{1}\right\|_{L^{2}}^{2}-\frac{\gamma G \bar{A}_{1}}{6}\left\|\eta_{1}^{2}\right\|_{L^{2}}^{2} \\
& -\frac{5 \gamma G \bar{A}_{2}}{12}\left\|\eta_{2}\right\|_{L^{2}}^{2}-\frac{\gamma G \bar{A}_{2}}{6}\left\|\eta_{2}^{2}\right\|_{L^{2}}^{2}-\frac{5 \gamma E A}{12}\|\varepsilon\|_{L^{2}}^{2}+A_{113}^{0} \\
& -\frac{\gamma E A}{6}\left\|\varepsilon^{2}\right\|_{L^{2}}^{2}-\gamma\langle\boldsymbol{n}, \boldsymbol{\mu} \times \mathbb{D} \boldsymbol{r} s\rangle_{L^{2}}+\gamma P_{0}\left\langle 1, \mathbb{D} \tilde{z}+\mathbb{D}^{2} \tilde{z} s\right\rangle_{L^{2}} \\
& -\gamma P_{0}\langle 1, \varepsilon+\mathbb{D} \varepsilon s\rangle_{L^{2}}-\gamma P_{0}\left\langle 1,1-\cos \left(\theta_{1}\right) \cos \left(\theta_{2}\right)\right\rangle_{L^{2}}
\end{aligned}
$$

where we have used the last equation of (24), and

$$
\begin{aligned}
A_{113}^{0} & =-\gamma E A\left\langle\varepsilon-\frac{1}{2} \varepsilon^{2}+\frac{1}{3} \varepsilon^{3}, 1-\cos \left(\theta_{1}\right) \cos \left(\theta_{2}\right)\right\rangle_{L^{2}} \\
& \leq \gamma E A \epsilon_{13}\|\varepsilon\|_{L^{2}}^{2}+\frac{\gamma E A\left(2 \epsilon_{14}+\epsilon_{14}^{\frac{4}{3}}\right)}{4}\left\|\varepsilon^{2}\right\|_{L^{2}}^{2} \\
& +\left[\frac{\gamma E A}{2 \epsilon_{13}}+\frac{\gamma E A}{4 \epsilon_{14}}+\frac{2 \gamma E A}{\epsilon_{14}^{4}}\right]\left\langle 1,1-\cos \left(\theta_{1}\right) \cos \left(\theta_{2}\right)\right\rangle_{L^{2}},
\end{aligned}
$$

with $\epsilon_{13}$ and $\epsilon_{14}$ being positive constants to be chosen. Substituting (B.17) into (B.16) gives

$$
\begin{aligned}
& A_{113} \leq \gamma \Gamma\left(\boldsymbol{n}^{B \Gamma}-P_{0} \boldsymbol{e}_{3}\right) \mathbb{D} \tilde{\boldsymbol{r}}^{B \Gamma}-\frac{5 \gamma \Gamma}{12}\left[G \bar{A}_{1} \wedge G \bar{A}_{2} \wedge E A\right] \\
& \times\left\|\boldsymbol{\vartheta}^{B \Gamma}\right\|^{2}-\gamma \Gamma P_{0}\left(\varepsilon^{B \Gamma}-\mathbb{D} \tilde{z}^{B \Gamma}\right)-\frac{5 \gamma G \bar{A}_{1}}{12}\left\|\eta_{1}\right\|_{L^{2}}^{2} \\
& -\frac{\gamma G \bar{A}_{1}}{6}\left\|\eta_{1}^{2}\right\|_{L^{2}}^{2}-\frac{5 \gamma G \bar{A}_{2}}{12}\left\|\eta_{2}\right\|_{L^{2}}^{2}-\frac{\gamma G \bar{A}_{2}}{6}\left\|\eta_{2}^{2}\right\|_{L^{2}}^{2} \\
& -\gamma E A\left(\frac{5}{12}-\epsilon_{13}\right)\|\varepsilon\|_{L^{2}}^{2}-\gamma E A\left[\frac{1}{6}-\frac{2 \epsilon_{14}+\epsilon_{14}^{3}}{4}\right]\left\|\varepsilon^{2}\right\|_{L^{2}}^{2} \\
& -\gamma\langle\boldsymbol{n},(\boldsymbol{\mu} \times \mathbb{D} \boldsymbol{r}) s\rangle_{L^{2}}-\gamma\left[P_{0}-\frac{\gamma E A}{2 \epsilon_{13}}-\frac{\gamma E A}{4 \epsilon_{14}}-\frac{2 \gamma E A}{\epsilon_{14}^{4}}\right] \\
& \times\left\langle 1,1-\cos \left(\theta_{1}\right) \cos \left(\theta_{2}\right)\right\rangle_{L^{2}},
\end{aligned}
$$

where we have used the fact that $\gamma P_{0}\left\langle 1, \mathbb{D} \tilde{z}+\mathbb{D}^{2} \tilde{z} s\right\rangle_{L^{2}}-$ $\gamma P_{0}\langle 1, \varepsilon+\mathbb{D} \varepsilon s\rangle_{L^{2}}=-\gamma \Gamma P_{0}\left(\varepsilon^{B \Gamma}-\mathbb{D} \tilde{z}^{B \Gamma}\right)$. 
The term $A_{114}$ : Using Young's and Hölder's inequalities, we can bound the term $A_{114}$ as follows:

$$
\begin{aligned}
& A_{114} \leq \gamma \Gamma\left[\lambda_{M}\left(\left|\boldsymbol{M}_{A}\right|\right) \epsilon_{15}+\lambda_{M}\left(\left|\boldsymbol{D}_{A 11}\right|\right) \epsilon_{16}\right]\|\boldsymbol{\vartheta}\|_{L^{2}}^{2} \\
& +\frac{\gamma \Gamma \lambda_{M}\left(\left|\boldsymbol{M}_{A}\right|\right)}{8 \epsilon_{15}}\left\||\boldsymbol{\omega}|^{2}\right\|_{L^{2}}^{2}+\frac{3 \gamma \Gamma \lambda_{M}\left(\left|\boldsymbol{D}_{A 12}\right| \epsilon_{17}^{3}\right)}{4}\left\||\boldsymbol{\vartheta}|^{2}\right\|_{L^{2}}^{2} \\
& +\left[\frac{\gamma \Gamma \lambda_{M}\left(\left|\boldsymbol{M}_{A}\right|\right)}{8 \epsilon_{15}}+\frac{\gamma \Gamma \lambda_{M}\left(\left|\boldsymbol{D}_{A 12}\right|\right)}{4 \epsilon_{17}^{4}}\right]\left\||\boldsymbol{v}|^{2}\right\|_{L^{2}}^{2} \\
& +\frac{\gamma \Gamma \lambda_{M}\left(\left|\boldsymbol{D}_{A 11}\right|\right)}{4 \epsilon_{16}}\|\boldsymbol{v}\|_{L^{2}}^{2},
\end{aligned}
$$

where $\epsilon_{1 i}, i=5,6,7$ are positive constants.

The term $A_{115}$ : Substituting $\boldsymbol{f}_{1}$ in (12) into $A_{115}$ and using Young's and Hölder's inequalities, we have

$$
\begin{aligned}
& A_{115} \leq \gamma \Gamma\left[\lambda_{M}\left(\left|\boldsymbol{M}_{A}\right|\right)\left(\epsilon_{18} \bar{v}_{f}^{M}+\epsilon_{19} \bar{\omega}_{f}^{M}\right)+3 \lambda_{M}\left(\left|\boldsymbol{D}_{A 12}\right|\right) \bar{v}_{f}^{M}\right. \\
& \left.\times\left(\epsilon_{110}+3 \epsilon_{111} \bar{v}_{f}^{M}\right)+\epsilon_{112}\right]\|\boldsymbol{\vartheta}\|_{L^{2}}^{2}+\frac{\gamma \Gamma \lambda_{M}\left(\left|\boldsymbol{M}_{A}\right| \bar{v}_{f}^{M}\right)}{4 \epsilon_{18}}\|\boldsymbol{\omega}\|_{L^{2}}^{2} \\
& +\gamma \Gamma\left[\frac{\lambda_{M}\left(\left|\boldsymbol{M}_{A}\right|\right) \bar{\omega}_{f}^{M}}{4 \epsilon_{19}}+\frac{3 \lambda_{M}\left(\left|\boldsymbol{D}_{A 12}\right|\left(\bar{v}_{f}^{M}\right)^{2}\right)}{4 \epsilon_{11}}\right]\|\boldsymbol{v}\|_{L^{2}}^{2} \\
& +\frac{\gamma \Gamma \lambda_{M}\left(\left|\boldsymbol{D}_{A 12}\right|\right) \bar{v}_{f}^{M}}{4 \epsilon_{110}}\left\||\boldsymbol{v}|^{2}\right\|_{L^{2}}^{2}+\frac{\gamma \Gamma}{4 \epsilon_{112}}\left\|\boldsymbol{f}_{10}\right\|_{L^{2}}^{2},
\end{aligned}
$$

where $\epsilon_{1 i}, i=8, \ldots, 12$ are positive constants. Substituting (B.13), (B.14), (B.18), (B.19), and (B.20) into (B.11) and using the last inequality in (24) results in (55).

\section{B.2.2 Calculation of $\mathcal{L} U_{12}$}

Applying Itô's formula to $U_{12}$ yields

$$
\mathcal{L} U_{12}=\sum_{i=1}^{5} A_{12 i}+\gamma\langle\boldsymbol{\mu}, \mathbb{D} \boldsymbol{r} \times \boldsymbol{n}\rangle_{L^{2}},
$$

with

$$
\begin{aligned}
& A_{121}=\gamma\left\langle\mu_{1 t} \boldsymbol{b}_{1}+\mu_{2 t} \boldsymbol{b}_{2}+\mu_{3 t} \boldsymbol{b}_{3}, \boldsymbol{J} \boldsymbol{\omega} s\right\rangle_{L^{2}}, \\
& A_{122}=2 \gamma\langle(\boldsymbol{\omega} \times \boldsymbol{\mu}), \boldsymbol{J} \boldsymbol{\omega} s\rangle_{L^{2}}, A_{123}=\gamma\langle\boldsymbol{\mu}, \mathbb{D} \boldsymbol{m} s\rangle_{L^{2}}, \\
& A_{124}=\gamma\left\langle\boldsymbol{\mu},\left(\boldsymbol{v} \times\left(\boldsymbol{M}_{A} \boldsymbol{v}\right)+\boldsymbol{D}_{A 21} \boldsymbol{\omega}+\boldsymbol{D}_{A 22} \boldsymbol{\omega} \otimes \boldsymbol{\omega} \boldsymbol{\omega}\right) s\right\rangle_{L^{2}}, \\
& A_{125}=\gamma\left\langle\boldsymbol{\mu}, \boldsymbol{f}_{2} s\right\rangle_{L^{2}},
\end{aligned}
$$

where we have used $\boldsymbol{\mu}_{t}=\mu_{1 t} \boldsymbol{b}_{1}+\mu_{2 t} \boldsymbol{b}_{2}+\mu_{3 t} \boldsymbol{b}_{3}+\boldsymbol{\omega} \times \boldsymbol{\mu}$ and $(\boldsymbol{\omega} \times \boldsymbol{\mu})^{T}(\boldsymbol{J} \boldsymbol{\omega})=-\boldsymbol{\mu}^{T}(\boldsymbol{\omega} \times(\boldsymbol{J} \boldsymbol{\omega}))$.

The term $A_{121}$ : We first use (2), (4), and the second equation of (11), then integration by parts to obtain

$$
\begin{aligned}
& A_{121} \leq \frac{\gamma \Gamma \lambda_{M}(\boldsymbol{J})}{2}\left\|\boldsymbol{\omega}^{B \Gamma}\right\|^{2}-\frac{\gamma \lambda_{m}(\boldsymbol{J})}{2}\|\boldsymbol{\omega}\|_{L^{2}}^{2} \\
& \quad+2 \gamma \Gamma \lambda_{M}(\boldsymbol{J}) \epsilon_{21}\|\boldsymbol{\mu}\|_{L^{2}}^{2}+\frac{\gamma \Gamma \lambda_{M}(\boldsymbol{J})}{2 \epsilon_{21}}\left\||\boldsymbol{\omega}|^{2}\right\|_{L^{2}}^{2},
\end{aligned}
$$

where $\epsilon_{21}$ is a positive constant to be determined.

The term $A_{122}$ : Applying Young's inequality yields

$$
A_{122} \leq \gamma \Gamma \lambda_{M}(\boldsymbol{J}) \epsilon_{22}\|\boldsymbol{\mu}\|_{L^{2}}^{2}+\frac{\gamma \Gamma \lambda_{M}(\boldsymbol{J})}{4 \epsilon_{22}}\left\||\boldsymbol{\omega}|^{2}\right\|_{L^{2}}^{2},
$$

where $\epsilon_{22}$ is a positive constant to be determined.

The term $A_{123}$ : Using integration by parts, $\mathbb{D} \boldsymbol{\mu}=$ $\mathbb{D} \mu_{1} \boldsymbol{b}_{1}+\mathbb{D} \mu_{2} \boldsymbol{b}_{2}+\mathbb{D} \mu_{3} \boldsymbol{b}_{3}$, and the expression of $\boldsymbol{m}$, see (14) and (15) yields

$$
\begin{gathered}
A_{123}=\gamma \Gamma\left(\boldsymbol{m}^{B \Gamma}\right)^{T} \boldsymbol{\mu}^{B \Gamma}-\frac{5 \gamma \Gamma}{12}\left[E I_{1} \wedge E I_{2} \wedge G I_{3}\right]\left\|\boldsymbol{\mu}^{B \Gamma}\right\|^{2} \\
\quad-\frac{5 \gamma E I_{1}}{12}\left\|\mu_{1}\right\|_{L^{2}}^{2}-\frac{5 \gamma E I_{2}}{12}\left\|\mu_{2}\right\|_{L^{2}}^{2}-\frac{5 \gamma G I_{3}}{12}\left\|\mu_{3}\right\|_{L^{2}}^{2} \\
-\frac{\gamma E I_{1}}{6}\left\|\mu_{1}^{2}\right\|_{L^{2}}^{2}-\frac{\gamma E I_{2}^{2}}{6}\left\|\mu_{2}^{2}\right\|_{L^{2}}^{2}-\frac{\gamma G I_{3}}{6}\left\|\mu_{3}^{2}\right\|_{L^{2}}^{2}
\end{gathered}
$$

The term $A_{124}$ : Applying Young's inequality gives

$$
\begin{aligned}
& A_{124} \leq \gamma \Gamma\left(\lambda_{M}\left(\left|\boldsymbol{M}_{A}\right|\right) \epsilon_{23}+\lambda_{M}\left(\left|\boldsymbol{D}_{A 21}\right|\right) \epsilon_{24}\right)\|\boldsymbol{\mu}\|_{L^{2}}^{2} \\
& +\frac{\gamma \Gamma \lambda_{M}\left(\left|\boldsymbol{M}_{A}\right|\right)}{4 \epsilon_{23}}\left\||\boldsymbol{v}|^{2}\right\|_{L^{2}}^{2}+\frac{\gamma \Gamma \lambda_{M}\left(\left|\boldsymbol{D}_{A 21}\right|\right)}{4 \epsilon_{24}}\|\boldsymbol{\omega}\|_{L^{2}}^{2} \\
& +\frac{\gamma \Gamma \lambda_{M}\left(\left|\boldsymbol{D}_{A 22}\right|\right) \epsilon_{25}^{\frac{4}{3}}}{4}\left\||\boldsymbol{\mu}|^{2}\right\|_{L^{2}}^{2}+\frac{\gamma \Gamma \lambda_{M}\left(\left|\boldsymbol{D}_{A 22}\right|\right)}{4 \epsilon_{25}^{4}}\left\||\boldsymbol{\omega}|^{2}\right\|_{L^{2}}^{2},
\end{aligned}
$$

where $\epsilon_{2 i}, i=3,4,5$ are positive constants to be chosen.

The term $A_{125}$ : Substituting $\boldsymbol{f}_{2}$ in (12) into $A_{125}$, and applying Young's and Hölder's inequalities results in

$$
\begin{aligned}
& A_{125} \leq \gamma \Gamma\left[2 \lambda_{M}\left(\left|\boldsymbol{J}_{A}\right|\right) \bar{\omega}_{f}^{M} \epsilon_{26}+2 \lambda_{M}\left(\left|\boldsymbol{M}_{A}\right|\right) \bar{v}_{f}^{M} \epsilon_{27}\right. \\
& +3 \lambda_{M}\left(\left|\boldsymbol{D}_{A 22}\right|\right) \bar{\omega}_{f}^{M} \epsilon_{28}+3 \lambda_{M}\left(\left|\boldsymbol{D}_{A 22}\right|\right)\left(\bar{\omega}_{f}^{M}\right)^{2} \epsilon_{29} \\
& \left.+\epsilon_{210}\right]\|\boldsymbol{\mu}\|_{L^{2}}^{2}+\gamma \Gamma\left[\frac{\lambda_{M}\left(\left|\boldsymbol{J}_{A}\right|\right) \bar{\omega}_{f}^{M}}{2 \epsilon_{26}}+\frac{3 \lambda_{M}\left(\left|\boldsymbol{D}_{A 22}\right|\right)\left(\bar{\omega}_{f}^{M}\right)^{2}}{4 \epsilon_{29}}\right] \\
& \times\|\boldsymbol{\omega}\|_{L^{2}}^{2}+\frac{\gamma \Gamma}{4 \epsilon_{210}}\left\|\boldsymbol{f}_{20}\right\|_{L^{2}}^{2}+\frac{\gamma \Gamma \lambda_{M}\left(\left|\boldsymbol{M}_{A}\right|\right) \bar{v}_{f}^{M}}{2 \epsilon_{27}}\|\boldsymbol{v}\|_{L^{2}}^{2} \\
& +\frac{3 \gamma \Gamma \lambda_{M}\left(\left|\boldsymbol{D}_{A 22}\right|\right) \bar{\omega}_{f}^{M}}{4 \epsilon_{28}}\left\|\left.\boldsymbol{\omega}\right|^{2}\right\|_{L^{2}}^{2},
\end{aligned}
$$

where $\epsilon_{2 i}, i=6, \ldots, 10$ are positive constants. Substituting (B.23), (B.24), (B.25), (B.26), and (B.27) into (B.21) yields $(57)$

\section{References}

[1] R. A. Adams and J. J. F. Fournier. Sobolev Spaces. Academic Press, Oxford, UK, 2nd edition, 2003.

[2] C. Athisakul, T. Monprapussorn, and S. Chucheepsakul. A variational formulation for three-dimensional analysis of extensible marine riser transporting fluid. Ocean Engineering, 38:609-620, 2011.

[3] M. M. Bernitsas, J. E. Kokarakis, and A. Imron. Large deformation three-dimensional static analysis of deep water marine risers. Applied Ocean Research, 7:178-187, 1985.

[4] M. Böhm, M. Krstíc, S. Küchler, and O. Sawodny. Modelling and boundary control of a hanging rope immersed in water. Journal of Dynamic Systems, Measurement, and Control, 136:011006-1:011006-15, 2014.

[5] A. Cavallo, G. de Maria, and C. Nataleand S. Pirozzi. Active Control of Flexible Structures. Springer, London, 2010.

[6] B. Chentouf and J. M. Wang. On the stabilization of the diskbeam system via torque and direct strain feedback controls. IEEE Transactions on Automatic Control, 60:3006-3011, 2015.

[7] P. L. Chow. Stochastic Partial Differential Equations. Chapman \& Hall/CRC, Boca Raton, 2007.

[8] K. D. Do. Global stabilization of three-dimensional flexible marine risers by boundary control. Ocean Systems Engineering, 1(2):171-194, 2011.

[9] K. D. Do. Modeling and boundary control of translational and rotational motions of nonlinear slender beams in threedimensional space. Journal of Sound and Vibration, 389:123, 2016.

[10] K. D. Do. Stability of nonlinear stochastic distributed parameter systems and its applications. Journal of Dynamic Systems, Measurement, and Control, 138:101010-1:10101012, 2016.

[11] K. D. Do. Boundary control of slender beams under deterministic and stochastic loads. Journal of Dynamic Systems, Measurement, and Control, In Press, 2017. 
[12] K. D. Do. Stochastic boundary control design for extensible marine risers in three dimensional space. Automatica, 77:184197, 2017.

[13] K. D. Do and J. Pan. Boundary control of transverse motion of marine risers with actuator dynamics. Journal of Sound and Vibration, 318(4-5):768-791, 2008.

[14] K. D. Do and J. Pan. Boundary control of three-dimensional inextensible marine risers. Journal of Sound and Vibration, 327(3-5):299-321, 2009 .

[15] T. Endo, F. Matsuno, and Y. Jia. Boundary cooperative control by flexible timoshenko arms. Automatica, 81:377$389,2017$.

[16] A. C. Eringen. On the nonlinear vibration of elastic bars. Quarterly of Applied Mathematics, 9:361-369, 1952.

[17] M. P. Fard and S. I. Sagatun. Exponential stabilization of a transversely vibrating beam by boundary control via lyapunov's direct method. Journal of Dynamic Systems, Measurement, and Control, 123:195-200, 2001.

[18] T. I. Fossen. Marine Control Systems. Marine Cybernetics, Trondheim, Norway, 2002.

[19] L. Gawarecki and V. Mandrekar. Stochastic Differential Equations in Infinite Dimensions with Applications to Stochastic Partial Differential Equations. Springer, Berlin, 2011.

[20] B. Z. Guo and F. F. Jin. The active disturbance rejection and sliding mode control approach to the stabilization of the Euler-Bernoulli beam equation with boundary input disturbance. Automatica, 49:2911-2918, 2013.

[21] I. Gyongy and N. V. Krylov. On stochastic equations with respect to semimartingales I. Stochastics, 4:1-21, 1980.

[22] W. He and S. S. Ge. Vibration control of a flexible beam with output constraint. IEEE Transactions on Industrial Electronics, 62:5023-5030, 2015

[23] W. He, S. S. Ge, B. Voon, E. How, and Y. S. Choo. Dynamics and Control of Mechanical Systems in Offshore Engineering. Springer, London, 2014.

[24] W. He, T. Meng D. Huang, and X. Li. Adaptive boundary iterative learning control for an Euler-Bernoulli beam system with input constraint. IEEE Transactions on Neural Networks and Learning Systems, In press, 2017.

[25] W. He, S. Nie, T. Meng, and Y. J. Liu. Modeling and vibration control for a moving beam with application in a drilling riser. IEEE Transactions on Control Systems Technology, 25:1036-1043, 2017.

[26] W. He, C. Sun, and S. S. Ge. Top tension control of a flexible marine riser by using integral-barrier Lyapunov function. IEEE/ASME Transactions on Mechatronics, 20:497-505, 2015.

[27] J. Henikl, W. Kemmetmüller, T. Meurer, and A. Kugi. Infinite-dimensional decentralized damping control of largescale manipulators with hydraulic actuation. Automatica, 63:101-115, 2016.

[28] F. F. Jin and B. Z. Guo. Lyapunov approach to output feedback stabilization for the Euler-Bernoulli beam equation with boundary input disturbance. Automatica, 52:95-102, 2015.

[29] A. Kater and T. Meurer. Modelling and flatness-based motion planning for an interconnected flexible beam structure. IEEE 55th Conference on Decision and Control, pages 520-525, 2016.

[30] R. Khasminskii. Stochastic Stability of Differential Equations. S\&N International, Rockville MD, 1980.
[31] J. E. Kokarakis and M. M. Bernitsas. Nonlinear threedimensional dynamic analysis of marine risers. Journal of Energy Resources Technology, 109:105-111, 1987.

[32] M. Krstíc and A. Smyshlyaev. Adaptive control of PDEs. Annual Reviews in Control, 32:149-160, 2008.

[33] M. Krstíc and A. Smyshlyaev. Boundary Control of PDEs: A Course on Backstepping Designs. Society for Industrial and Applied Mathematics, Philadelphia, PA, 2008.

[34] J. B. Kuipers. Quaternions and Rotation Sequences: A Primer with Applications to Orbits, Aerospace and Virtual Reality. Princeton University Press, New Jersey, 2002.

[35] J. E. Lagnese, G. Leugering, and E. J. P. G. Schmidt. Modeling, Analysis and Control of Dynamic Elastic MultiLink Structures. Birkhäuser, Boston, 1994.

[36] K. Liu. Stability of infinite dimensional stochastic differential equations with applications. Chapman and Hall/CRC, Boca Raton, FL, 2006.

[37] A. Love. A Treatise on the Mathematical Theory of Elasticity. Cambridge University Press, 3rd edition, 1920.

[38] Z. H. Luo, B. Z. Guo, and O. Morgul. Stability and stabilization of infinite dimensional systems with applications. Springer-Verlag, 1999.

[39] T. Meurer, D. Thull, and A. Kugi. Flatness-based tracking control of a piezoactuated Euler-Bernoulli beam with non-collocated output feedback: theory and experiments. International Journal of Control, 81:475-493, 2008.

[40] M. Miletíc, D. Stürzer, A. Arnold, and A. Kugi. Stability of an Euler-Bernoulli beam with a nonlinear dynamic feedback system. IEEE Transactions on Automatic Control, 61:27822795, 2016.

[41] T. L. Nguyen, K. D. Do, and J. Pan. Boundary control of twodimensional marine risers with bending couplings. Journal of Sound and Vibration, 332(16):3605-3622, 2013.

[42] J. M. Niedzwecki and P. Y. F. Liagre. System identification of distributed-parameter marine riser models. Ocean Engineering, 30(11):1387-1415, 2003.

[43] W. C. Orthwein. A nonlinear stress-strain relation. International Journal of Solids and Structures, 4:371-382, 1968.

[44] A. O. Özer. Modeling and controlling an active constrained layer (acl) beam actuated by two voltage sources with/without magnetic effects. IEEE Transactions on Automatic Control, In press, 2017.

[45] E. Pardoux. Stochastic partial differential equations and filtering of diffusion processes. Stochastics, 3:127-167, 1979.

[46] G. Da Prato and J. Zabczyk. Stochastic Equations in Infinite Dimensions. Cambridge University Press, Cambridge, UK, 1992.

[47] C. Prévôt and M. Röckner. A Concise Course on Stochastic Partial Differential Equations. Springer, Berlin, 2007.

[48] M. S. De Queiroz, M. Dawson, S. Nagarkatti, and F. Zhang. Lyapunov-Based Control of Mechanical Systems. Birkhauser, Boston, 2000.

[49] T. Sarpkaya and M. Isaacso. Mechanics of Wave Forces on Offshore Structures. Van Nostrand Reinhold, New York, 1981.

[50] A. L. Zuyev. Partial Stabilization and Control of Distributed Parameter Systems with Elastic Elements. Springer International Publishing, Switzerland, 2015. 